\title{
CORRECTOR RESULTS FOR A PARABOLIC PROBLEM WITH A MEMORY EFFECT
}

\author{
Patrizia Donato ${ }^{1}$ and Editha C. Jose $^{2}$
}

\begin{abstract}
The aim of this paper is to provide the correctors associated to the homogenization of a parabolic problem describing the heat transfer. The results here complete the earlier study in [Jose, Rev. Roumaine Math. Pures Appl. 54 (2009) 189-222] on the asymptotic behaviour of a problem in a domain with two components separated by an $\varepsilon$-periodic interface. The physical model established in [Carslaw and Jaeger, The Clarendon Press, Oxford (1947)] prescribes on the interface the condition that the flux of the temperature is proportional to the jump of the temperature field, by a factor of order $\varepsilon^{\gamma}$. We suppose that $-1<\gamma \leq 1$. As far as the energies of the homogenized problems are concerned, we consider the cases $-1<\gamma<1$ and $\gamma=1$ separately. To obtain the convergence of the energies, it is necessary to impose stronger assumptions on the data. As seen in [Jose, Rev. Roumaine Math. Pures Appl. 54 (2009) 189-222] and [Faella and Monsurrò, Topics on Mathematics for Smart Systems, World Sci. Publ., Hackensack, USA (2007) 107-121] (also in [Donato et al., J. Math. Pures Appl. 87 (2007) 119-143]), the case $\gamma=1$ is more interesting because of the presence of a memory effect in the homogenized problem.
\end{abstract}

Mathematics Subject Classification. 35B27, 35K20, 82B24.

Received April 17, 2009. Revised September 3, 2009.

Published online February 4, 2010.

\section{INTRODUCTION}

This paper is devoted to the study of corrector results associated to the homogenization of the parabolic problem studied in [21]. In this work, the domain $\Omega \subset \mathbb{R}^{n}$ is given by $\Omega=\Omega_{1 \varepsilon} \cup \overline{\Omega_{2 \varepsilon}}$. By taking $Y=Y_{1} \cup \overline{Y_{2}}$ to be the reference cell, $\Omega_{1 \varepsilon}$ and $\Omega_{2 \varepsilon}$ are respectively, the connected and disconnected union of $\varepsilon$-periodic translated sets of $\varepsilon Y_{1}$ and $\varepsilon Y_{2}$. On the other hand, $\Gamma^{\varepsilon}:=\partial \Omega_{2 \varepsilon}$ is the interface separating the two components

\footnotetext{
Keywords and phrases. Periodic homogenization, correctors, heat equation, interface problems.

${ }^{1}$ Université de Rouen, Laboratoire de Mathématiques Raphaël Salem, UMR 6085 CNRS, Avenue de l'Université, BP 12, 76801 St Étienne du Rouvray, France. Patrizia.Donato@univ-rouen.fr

2 Math Division, Institute of Mathematical Sciences and Physics, College of Arts and Sciences, University of the Philippines Los Baños, Los Baños, Laguna, 4031, Philippines. ecjose@uplb.edu.ph
} 
with $\partial \Omega \cap \Gamma^{\varepsilon}=\emptyset$. For $T>0$ and $-1<\gamma \leq 1$, consider the following problem:

$$
\begin{cases}u_{1 \varepsilon^{\prime}}{ }^{\prime}-\operatorname{div}\left(A^{\varepsilon} \nabla u_{1 \varepsilon}\right)=f_{1 \varepsilon}+P_{1}^{\varepsilon^{*}}(g) & \text { in } \left.\Omega_{1 \varepsilon} \times\right] 0, T[, \\ u_{2 \varepsilon}{ }^{\prime}-\operatorname{div}\left(A^{\varepsilon} \nabla u_{2 \varepsilon}\right)=f_{2 \varepsilon} & \text { in } \left.\Omega_{2 \varepsilon} \times\right] 0, T[, \\ A^{\varepsilon} \nabla u_{1 \varepsilon} \cdot n_{1 \varepsilon}=-A^{\varepsilon} \nabla u_{2 \varepsilon} \cdot n_{2 \varepsilon} & \text { on } \left.\Gamma^{\varepsilon} \times\right] 0, T[, \\ A^{\varepsilon} \nabla u_{1 \varepsilon} \cdot n_{1 \varepsilon}=-\varepsilon^{\gamma} h^{\varepsilon}\left(u_{1 \varepsilon}-u_{2 \varepsilon}\right) & \text { on } \left.\Gamma^{\varepsilon} \times\right] 0, T[, \\ u_{1 \varepsilon}=0 & \text { on } \partial \Omega \times] 0, T[, \\ u_{1 \varepsilon}(x, 0)=U_{1 \varepsilon}^{0} \text { in } \Omega_{1 \varepsilon}, u_{2 \varepsilon}(x, 0)=U_{2 \varepsilon}^{0} \text { in } \Omega_{2 \varepsilon}, & \end{cases}
$$

where $n_{i \varepsilon}$ is the unitary outward normal to $\Omega_{i \varepsilon}(i=1,2), P_{1}^{\varepsilon}$ is a suitable extension operator and $P_{1}^{\varepsilon^{*}}$ its adjoint. The coefficient $A^{\varepsilon}$ is assumed to be independent of $t$, uniformly bounded in $L^{\infty}(\Omega)$ and satisfying the ellipticity condition given by (2.8)(i). Moreover, $h^{\varepsilon}$ is an oscillating periodic function which is bounded in $L^{\infty}\left(\Gamma^{\varepsilon}\right)$. Meanwhile, the data $f_{i \varepsilon}$ and $U_{i \varepsilon}^{0}(i=1,2)$, belongs to $L^{2}\left(0, T ; L^{2}(\Omega)\right)$ and $L^{2}\left(\Omega_{i \varepsilon}\right)$ respectively.

This paper completes the investigation of the asymptotic behaviour of a parabolic problem earlier considered by Faella and Monsurrò [19] and Jose [21]. Our aim is to find corrector results in order to improve the weak approximations from [21]. The notion of corrector matrix, which was introduced by Tartar in [30,31], plays an important role in homogenization theory.

Let us recall the convergence results from [21]. If $\theta_{i}=\frac{\left|Y_{i}\right|}{|Y|}(i=1,2)$ is the proportion of the material occupying $\Omega_{i \varepsilon}$ and

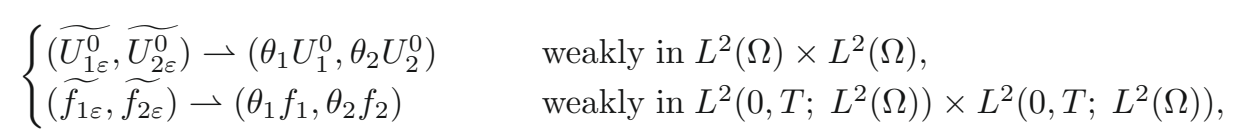

where $\sim$ denotes the zero extension to the whole of $\Omega$, then for all $-1<\gamma \leq 1$,

$$
\begin{cases}\left(\text { i) } P_{1}^{\varepsilon} u_{1 \varepsilon}-u_{1}\right. & \text { weakly in } L^{2}\left(0, T ; H_{0}^{1}(\Omega)\right), \\ \text { (ii) } \widetilde{u_{1 \varepsilon}}-\theta_{1} u_{1} & \text { weakly }{ }^{*} \text { in } L^{\infty}\left(0, T ; L^{2}(\Omega)\right), \\ (\text { iii }) \widetilde{u_{2 \varepsilon}}-u_{2} & \text { weakly* in } L^{\infty}\left(0, T ; L^{2}(\Omega)\right), \\ \text { (iv) } \varepsilon^{\frac{\gamma}{2}}\left\|u_{1 \varepsilon}-u_{2 \varepsilon}\right\|_{L^{2}\left(0, T ; L^{2}\left(\Gamma^{\varepsilon}\right)\right)}<c . & \end{cases}
$$

Furthermore,

$$
\begin{cases}\text { (i) } A^{\varepsilon} \widetilde{\nabla u_{1 \varepsilon}}-A^{0} \nabla u_{1} & \text { weakly in } L^{2}\left(0, T ;\left[L^{2}(\Omega)\right]^{n}\right), \\ \text { (ii) } A^{\varepsilon} \widetilde{\nabla u_{2 \varepsilon}}-0 & \text { weakly in } L^{2}\left(0, T ;\left[L^{2}(\Omega)\right]^{n}\right),\end{cases}
$$

where $A^{0}$ is the homogenized matrix obtained by Cioranescu and Saint Jean Paulin in [9], for the Laplace problem in a perforated domain with a Neumann condition on the boundary of the holes.

The homogenized (limit) problems satisfied by the couple $\left(u_{1}, u_{2}\right)$ are different for the two cases $-1<\gamma<1$ and $\gamma=1$. We describe first the case $-1<\gamma<1$, where $u_{2}=\theta_{2} u_{1}$ and $u_{1}$ is the unique solution of the homogenized problem

$$
\begin{cases}u_{1}^{\prime}-\operatorname{div}\left(A^{0} \nabla u_{1}\right)=\theta_{1} f_{1}+\theta_{2} f_{2}+g & \text { in } \Omega \times] 0, T[ \\ u_{1}=0 & \text { on } \partial \Omega \times] 0, T[, \\ u_{1}(0)=\theta_{1} U_{1}^{0}+\theta_{2} U_{2}^{0} & \text { in } \Omega .\end{cases}
$$


For this case, the corrector result given in Theorem 3.4 and proved in Section 6 states the following convergences:

$$
\left\{\begin{array}{l}
\left(\text { i) } \widetilde{u_{1 \varepsilon}}+\widetilde{u_{2 \varepsilon}} \rightarrow u_{1} \quad \text { in } \mathcal{C}^{0}\left([0, T] ; L^{2}(\Omega)\right),\right. \\
\text { (ii) } \lim _{\varepsilon \rightarrow 0}\left\|\nabla u_{1 \varepsilon}-C^{\varepsilon} \nabla u_{1}\right\|_{L^{2}\left(0, T ;\left[L^{1}\left(\Omega_{1 \varepsilon}\right)\right]^{n}\right)}=0, \\
\text { (iii) } \lim _{\varepsilon \rightarrow 0}\left\|\nabla u_{2 \varepsilon}\right\|_{L^{2}\left(0, T ;\left[L^{2}\left(\Omega_{2 \varepsilon}\right)\right]^{n}\right)}=0,
\end{array}\right.
$$

where $\left(u_{1 \varepsilon}, u_{2 \varepsilon}\right)$ is the solution of problem (1.1) and $C^{\varepsilon}$ is the corrector matrix associated with $A^{0}$.

To prove that result, stronger assumptions on the data than (1.2) are necessary, in order to establish the convergence of the energy of the $\varepsilon$-problem to that of the homogenized one. That was also the case in the homogenization of the wave and heat equations in a fixed domain $\Omega$ done by Bensoussan et al. in [2] and Brahim-Otsman et al. in [3] (see also [11]).

For the first case $-1<\gamma<1$, we make here the stronger assumptions that $f_{i \varepsilon} \in L^{2}\left(0, T ; L^{2}(\Omega)\right), U_{i \varepsilon}^{0} \in$ $L^{2}\left(\Omega_{i \varepsilon}\right)(i=1,2)$, and satisfy

$$
\begin{cases}(i) f_{i \varepsilon} \rightarrow f_{i} & \text { strongly in } L^{2}\left(0, T ; L^{2}(\Omega)\right), \\ (i i) \widetilde{U_{1 \varepsilon}^{0}}+\widetilde{U_{2 \varepsilon}^{0}} \rightarrow U^{0} & \text { strongly in } L^{2}(\Omega) .\end{cases}
$$

To describe the corrector results for the case $\gamma=1$, we recall from [21] that $\left(u_{1}, u_{2}\right)$ is the unique solution of the coupled system

$$
\begin{cases}\theta_{1} u_{1}^{\prime}-\operatorname{div}\left(A^{0} \nabla u_{1}\right)+c_{h}\left(\theta_{2} u_{1}-u_{2}\right)=\theta_{1} f_{1}+g & \text { in } \Omega \times] 0, T[, \\ u_{2}^{\prime}-c_{h}\left(\theta_{2} u_{1}-u_{2}\right)=\theta_{2} f_{2} & \text { in } \Omega \times] 0, T[, \\ u_{1}=0 & \text { on } \partial \Omega \times] 0, T[, \\ u_{1}(0)=U_{1}^{0}, u_{2}(0)=\theta_{2} U_{2}^{0} & \text { in } \Omega,\end{cases}
$$

where $c_{h}=\frac{1}{\left|Y_{2}\right|} \int_{\Gamma} h(y) \mathrm{d} \sigma_{y}$. Solving the ODE in (1.5) and replacing $u_{2}$ in the PDE, shows that $u_{1}$ satisfies an equation of the form

$$
\theta_{1} u_{1}^{\prime}-\operatorname{div}\left(A_{\gamma}^{0} \nabla u_{1}\right)+c_{h} \theta_{2} u_{1}-c_{h}^{2} \theta_{2} \int_{0}^{t} \mathcal{K}(t, s) u_{1}(s) \mathrm{d} s=F(x, t),
$$

with $\mathcal{K}$ an exponential kernel, giving rise to a memory effect.

We now introduce the stronger assumptions on the data. We suppose that for $f_{i \varepsilon} \in L^{2}\left(0, T ; L^{2}(\Omega)\right)$ and $U_{i \varepsilon}^{0} \in L^{2}\left(\Omega_{i \varepsilon}\right)(i=1,2)$, one has

$$
\left\{\begin{array}{l}
(i) f_{i \varepsilon} \rightarrow f_{i} \quad \text { strongly in } L^{2}\left(0, T ; L^{2}(\Omega)\right) \\
(\text { ii }) U_{i \varepsilon}^{0} \rightarrow \theta_{i} U_{i}^{0} \quad \text { weakly in } L^{2}(\Omega), \\
\left(\text { iii }\left\|U_{1 \varepsilon}^{0}\right\|_{L^{2}\left(\Omega_{1 \varepsilon}\right)}^{2}+\left\|U_{2 \varepsilon}^{0}\right\|_{L^{2}\left(\Omega_{2 \varepsilon}\right)}^{2} \rightarrow \theta_{1}\left\|U_{1}^{0}\right\|_{L^{2}(\Omega)}^{2}+\theta_{2}\left\|U_{2}^{0}\right\|_{L^{2}(\Omega)}^{2} .\right.
\end{array}\right.
$$

Then, assuming that $\Gamma$ is of class $C^{2}$, the following corrector results for the case $\gamma=1$ hold true:

$$
\left\{\begin{array}{l}
\left(\text { i) } \lim _{\varepsilon \rightarrow 0}\left\|u_{1 \varepsilon}-u_{1}\right\|_{\mathcal{C}^{0}\left(0, T ; L^{2}\left(\Omega_{1 \varepsilon}\right)\right)}=0\right. \\
(\text { ii }) \lim _{\varepsilon \rightarrow 0}\left\|u_{2 \varepsilon}-\theta_{2}^{-1} u_{2}\right\|_{\mathcal{C}^{0}\left(0, T ; L^{2}\left(\Omega_{2 \varepsilon}\right)\right)}=0 \\
\text { (iii) } \lim _{\varepsilon \rightarrow 0}\left\|\nabla u_{1 \varepsilon}-C^{\varepsilon} \nabla u_{1}\right\|_{L^{2}\left(0, T ;\left[L^{1}\left(\Omega_{1 \varepsilon}\right)\right]^{n}\right)}=0 \\
\text { (iv) } \lim _{\varepsilon \rightarrow 0}\left\|\nabla u_{2 \varepsilon}\right\|_{L^{2}\left(0, T ;\left[L^{2}\left(\Omega_{2 \varepsilon}\right)\right]^{n}\right)}=0
\end{array}\right.
$$


As seen in Section 5, assumptions (1.4) and assumptions (1.6) are well adapted to the homogenized problems (1.3) and (1.5).

In both cases, the proof of the main results are based on a suitable upper semicontinuity-type inequality. Despite the fact that this approach is classical, we have a specific difficulty in the parabolic case because of the influence of the interface. Indeed, some compactness of the solution $\left(u_{1 \varepsilon}, u_{2 \varepsilon}\right)$ in a space of type $\mathcal{C}^{0}([0, T] ; X)$ is needed. In the classical case of a fixed domain, such a compactness of the solution in $\mathcal{C}^{0}\left([0, T] ; L^{2}(\Omega)\right)$ is straightforward. This is not true in perforated domains. In this case, a compactness result in $\mathcal{C}^{0}\left([0, T] ; H^{-1}(\Omega)\right)$ was proved in [15], leading to a corrector result. The situation here is complicated by the fact that we have the couple of functions $u_{1 \varepsilon}, u_{2 \varepsilon}$. Nevertheless, we are able to prove that the sequence $\left\{\widetilde{u_{1 \varepsilon}}+\widetilde{u_{2 \varepsilon}}\right\}$ is compact in $\mathcal{C}^{0}\left([0, T] ; H^{-1}(\Omega)\right)$ (see Thm. 4.8). Moreover, for $\gamma=1$, the sequences $\left\{\widetilde{u_{1 \varepsilon}}\right\}$ and $\left\{\widetilde{u_{2 \varepsilon}}\right\}$ are also separately compact in $\mathcal{C}^{0}\left([0, T] ; H^{-1}(\Omega)\right)$. These compactness results play a crucial role when proving the corrector results.

For the case $\gamma=1$ (see Step 2 of the proof of Prop. 6.8), we had to adapt to the parabolic case some technical lemmas for the elliptic and hyperbolic case $[12,18]$. In contrast to the hyperbolic case, the coefficient matrix $A^{\varepsilon}$ is not necessarily symmetric in our case and this is a significant difference. Indeed, when proving the upper semicontinuity-type inequality there is an additional term (in both cases), which needs specific arguments (see Step 1.3 and Step 1 in Sects. 6.1 and 6.2). We refer to Remarks 6.6, 6.7 and 6.9 for more details on these technical points.

This paper is organized as follows. In Section 2, we recall the geometric and functional setting of the problem together with the homogenization results proved in [21]. In Section 3, the corrector results are stated as well as the necessary assumptions regarding the data. We also give a detailed description of these assumptions. In Section 4, we investigate the compactness of $\widetilde{u_{1 \varepsilon}}+\widetilde{u_{2 \varepsilon}}$ in $\mathcal{C}^{0}\left([0, T] ; H^{-1}(\Omega)\right)$ discussed above. Section 5 is devoted to the convergence of the energy of the $\varepsilon$-problems. Finally, in Section 6 we prove the corrector results stated in Section 3.

The homogenization of elliptic and hyperbolic problems in a domain with the same geometric and abstract framework as in the present paper, were already done by Monsurrò [26,27], Donato and Monsurrò [13], and Donato et al. $[17,18]$. For similar studies of problems with jump conditions in the elliptic case we refer to $[1,20,23,24]$ and the references therein. Our results can be related to the case of parabolic problems in perforated domains that were studied by Donato and Nabil [15]. The homogenization of Neumann boundary problems in perforated domains were investigated by Cioranescu and Saint Jean Paulin in $[9,10]$. For associated correctors we refer to Donato et al. [16]. For the pioneer works on linear memory effects in the homogenization of parabolic problems, we refer to Mascarenhas in [25] and Tartar in [32]. For other homogenization of parabolic and hyperbolic problems for which memory effects occur, we also refer to the recent articles [28,29].

\section{Preliminaries}

We recall the geometric framework used for the homogenization of problem (1.1) in [21]. We consider an open bounded set $\Omega$ of $\mathbb{R}^{n}$ which is decomposed into the connected set $\Omega_{1 \varepsilon}$ and the disconnected set $\Omega_{2 \varepsilon}$, both of which are unions of $\varepsilon^{-n}$ translated sets, with $\{\varepsilon\}$ a sequence of positive real numbers that converges to zero.

Let $Y=] 0, \ell_{1}[\times \ldots \times] 0, \ell_{n}\left[\right.$ and let $Y_{1}$ and $Y_{2}$ be two nonempty open sets such that $Y=Y_{1} \cup \overline{Y_{2}}$. We suppose that $Y_{1}$ is connected and $Y_{2}$ has a Lipschitz continuous boundary $\Gamma$.

For any $k \in \mathbb{Z}^{n}, Y_{i}^{k}$ and $\Gamma_{k}$ are the translated sets

$$
Y_{i}^{k}:=k_{\ell}+Y_{i}, \quad \Gamma_{k}:=k_{\ell}+\Gamma \quad \text { where } \quad k_{\ell}=\left(k_{1} \ell_{1}, \ldots, k_{n} \ell_{n}\right) \quad \text { and } \quad i=1,2 .
$$

For any given $\varepsilon$, set

$$
K_{\varepsilon}:=\left\{k \in \mathbb{Z}^{n} \mid \varepsilon Y_{i}^{k} \cap \Omega \neq \emptyset, i=1,2\right\} .
$$

We then define the two components of $\Omega$ and the interface respectively as follows:

$$
\Omega_{i \varepsilon}:=\Omega \cap\left\{\bigcup_{k \in K_{\varepsilon}} \varepsilon Y_{i}^{k}\right\}, \quad i=1,2 \quad \text { and } \quad \Gamma^{\varepsilon}=\partial \Omega_{2 \varepsilon} .
$$


Assume that

$$
\partial \Omega \cap\left(\bigcup_{k \in \mathbb{Z}^{n}}\left(\varepsilon \Gamma_{k}\right)\right)=\emptyset,
$$

and so $\partial \Omega \cap \Gamma^{\varepsilon}=\emptyset$.

Remark 2.1. The above geometric assumption is the one used in the homogenization of the parabolic problem (1.1). This gives a simpler presentation, as it was the case in the hyperbolic $[17,18]$ and elliptic cases [12, 13,26,27]. Assumption (2.1) can be replaced by a different definition of $\Omega_{2 \varepsilon}$ as the union of all the set $\varepsilon Y_{2}^{k}$ such that $\overline{\varepsilon Y_{2}^{k}} \subset \Omega$. In such a case, all the previous results and those proved here are still true.

In the sequel, we will use the following notation:

- $\chi_{\omega}$ the characteristic function of any open set $\omega \subset \mathbb{R}^{n}$;

- $m_{\omega}(v)=\frac{1}{|\omega|} \int_{\omega} v \mathrm{~d} x$, the mean value of $v$ over a measurable set $\omega$;

- $\widetilde{v}$ the zero extension to $\mathbb{R}^{n}$ of any function $v$ defined on $\Omega_{i \varepsilon}$ or $Y_{i}$ for $i=1,2$.

Remark 2.2. To simplify notation, if a function $v$ is defined on the whole of $\Omega$, we still denote by $v$ its restriction to $\Omega_{i \varepsilon}$ when no confusion arises. We will also use the fact that

$$
\widetilde{\left.v\right|_{\Omega_{i \varepsilon}}}=\chi_{\Omega_{i \varepsilon}} v, \quad \text { for } i=1,2 .
$$

It is known that (for instance, see [8]),

$$
\chi_{\Omega_{i \varepsilon}} \rightarrow \theta_{i}:=\frac{\left|Y_{i}\right|}{|Y|}(i=1,2), \text { weakly in } L^{2}(\Omega) .
$$

We consider the two spaces $V^{\varepsilon}$ and $H_{\gamma}^{\varepsilon}$ defined by

$$
\begin{gathered}
V^{\varepsilon}:=\left\{v_{1} \in H^{1}\left(\Omega_{1 \varepsilon}\right) \mid v_{1}=0 \text { on } \partial \Omega\right\}, \\
H_{\gamma}^{\varepsilon}:=\left\{v=\left(v_{1}, v_{2}\right) \mid v_{1} \in V^{\varepsilon} \text { and } v_{2} \in H^{1}\left(\Omega_{2 \varepsilon}\right)\right\}, \quad \forall \gamma \in \mathbb{R},
\end{gathered}
$$

which are Banach spaces respectively, for the norms

$$
\left\|v_{1}\right\|_{V^{\varepsilon}}:=\left\|\nabla v_{1}\right\|_{L^{2}\left(\Omega_{1 \varepsilon}\right)}
$$

and

$$
\|v\|_{H_{\gamma}^{\varepsilon}}^{2}:=\left\|\nabla v_{1}\right\|_{L^{2}\left(\Omega_{1 \varepsilon}\right)}^{2}+\left\|\nabla v_{2}\right\|_{L^{2}\left(\Omega_{2 \varepsilon}\right)}^{2}+\varepsilon^{\gamma}\left\|v_{1}-v_{2}\right\|_{L^{2}\left(\Gamma^{\varepsilon}\right)}^{2} .
$$

Remark 2.3. As already seen in $[9,10]$, a uniform Poincaré inequality holds in $V^{\varepsilon}$, i.e., there exists a constant $C>0$ (independent of $\varepsilon$ ) such that for every $\varepsilon$

$$
\|v\|_{L^{2}\left(\Omega_{1 \varepsilon}\right)} \leq C\|\nabla v\|_{L^{2}\left(\Omega_{1 \varepsilon}\right)}, \quad \forall v \in V^{\varepsilon} .
$$

On the other hand, observe that if $\gamma_{1} \leq \gamma_{2}$ then

$$
\|v\|_{H_{\gamma_{2}}^{\varepsilon}}^{2} \leq\|v\|_{H_{\gamma_{1}}^{\varepsilon}}^{2} .
$$

Hence in particular, for all $\gamma \leq 1$ we have

$$
\|v\|_{H_{1}^{\varepsilon}} \leq\|v\|_{H_{\gamma}^{\varepsilon}} .
$$

Let us recall the following result from $[26,27]$ giving equivalence of norms. 
Lemma 2.4 [26]. There exist two positive constants $C_{1}, C_{2}$ (independent of $\varepsilon$ ) such that

$$
C_{1}\|v\|_{H_{1}^{\varepsilon}} \leq\|v\|_{V^{\varepsilon} \times H^{1}\left(\Omega_{2 \varepsilon}\right)} \leq C_{2}\|v\|_{H_{1}^{\varepsilon}}, \quad \forall v \in H_{1}^{\varepsilon}
$$

With this functional setting, we now can state our parabolic problem.

Suppose that

$$
\left\{\begin{array}{l}
g \in L^{2}\left(0, T ; H^{-1}(\Omega)\right) \\
\left(U_{1 \varepsilon}^{0}, U_{2 \varepsilon}^{0}\right) \in L^{2}\left(\Omega_{1 \varepsilon}\right) \times L^{2}\left(\Omega_{2 \varepsilon}\right) \\
\left(f_{1 \varepsilon}, f_{2 \varepsilon}\right) \in L^{2}\left(0, T ; L^{2}(\Omega)\right) \times L^{2}\left(0, T ; L^{2}(\Omega)\right) .
\end{array}\right.
$$

Furthermore, let $A$ be a $n \times n$ matrix field in $\left(L^{\infty}(Y)\right)^{n^{2}}, Y$-periodic and such that $\forall \lambda \in \mathbb{R}^{n}$ and a.e. in $Y$,

$$
\left\{\begin{array}{l}
(i)(A(x) \lambda, \lambda) \geq \alpha|\lambda|^{2} \\
(i i)|A(x) \lambda| \leq \beta \lambda
\end{array}\right.
$$

where $\alpha, \beta \in \mathbb{R}$ with $0<\alpha<\beta$. For any $\varepsilon>0$, we set

$$
A^{\varepsilon}(x):=A\left(\frac{x}{\varepsilon}\right)
$$

We also suppose that $h$ is a $Y$-periodic function satisfying

$$
h \in L^{\infty}(\Gamma), \quad \exists h_{0} \in \mathbb{R} \quad \text { such that } 0<h_{0}<h(y), \quad y \quad \text { a.e. in } \Gamma
$$

and set

$$
h^{\varepsilon}(x):=h\left(\frac{x}{\varepsilon}\right)
$$

For $T>0$ and $-1<\gamma \leq 1$, consider the following problem:

$$
\begin{cases}u_{1 \varepsilon}{ }^{\prime}-\operatorname{div}\left(A^{\varepsilon} \nabla u_{1 \varepsilon}\right)=f_{1 \varepsilon}+P_{1}^{\varepsilon^{*}}(g) & \text { in } \left.\Omega_{1 \varepsilon} \times\right] 0, T[, \\ u_{2 \varepsilon}{ }^{\prime}-\operatorname{div}\left(A^{\varepsilon} \nabla u_{2 \varepsilon}\right)=f_{2 \varepsilon} & \text { in } \left.\Omega_{2 \varepsilon} \times\right] 0, T[, \\ A^{\varepsilon} \nabla u_{1 \varepsilon} \cdot n_{1 \varepsilon}=-A^{\varepsilon} \nabla u_{2 \varepsilon} \cdot n_{2 \varepsilon} & \text { on } \left.\Gamma^{\varepsilon} \times\right] 0, T[, \\ A^{\varepsilon} \nabla u_{1 \varepsilon} \cdot n_{1 \varepsilon}=-\varepsilon^{\gamma} h^{\varepsilon}\left(u_{1 \varepsilon}-u_{2 \varepsilon}\right) & \text { on } \left.\Gamma^{\varepsilon} \times\right] 0, T[, \\ u_{1 \varepsilon}=0 & \text { on } \partial \Omega \times] 0, T[, \\ u_{1 \varepsilon}(x, 0)=U_{1 \varepsilon}^{0} \text { in } \Omega_{1 \varepsilon}, u_{2 \varepsilon}(x, 0)=U_{2 \varepsilon}^{0} \text { in } \Omega_{2 \varepsilon}, & \end{cases}
$$

where $n_{i \varepsilon}$ is the unitary outward normal to $\Omega_{i \varepsilon}(i=1,2), P_{1}^{\varepsilon}$ is a suitable extension operator (see Lem. 4.5) and $P_{1}^{\varepsilon *}$ its adjoint.

By definition, for any $g \in L^{2}\left(0, T ; H^{-1}(\Omega)\right), P_{1}^{\varepsilon^{*}} g$ is given by

$$
P_{1}^{\varepsilon^{*}} g: v \in L^{2}\left(0, T ; V^{\varepsilon}\right) \longmapsto \int_{0}^{T}\left\langle g, P_{1}^{\varepsilon} v\right\rangle_{H^{-1}(\Omega), H_{0}^{1}(\Omega)} \mathrm{d} s .
$$

Observe that

$$
P_{1}^{\varepsilon *} \in \mathcal{L}\left(L^{2}\left(0, T ; H^{-1}(\Omega)\right) ; L^{2}\left(0, T ;\left(V^{\varepsilon}\right)^{\prime}\right)\right) .
$$


The variational formulation of problem (2.12) is

$$
\left\{\begin{array}{l}
\begin{array}{l}
\text { Find } u_{\varepsilon}=\left(u_{1 \varepsilon}, u_{2 \varepsilon}\right) \text { in } W^{\varepsilon} \text { such that } \\
\int_{0}^{T}\left\langle u_{1 \varepsilon}^{\prime}, v_{1}\right\rangle_{\left(V^{\varepsilon}\right)^{\prime}, V^{\varepsilon}} \mathrm{d} t+\int_{0}^{T}\left\langle u_{2 \varepsilon}^{\prime}, v_{2}\right\rangle_{\left(H^{1}\left(\Omega_{2 \varepsilon}\right)\right)^{\prime}, H^{1}\left(\Omega_{2 \varepsilon}\right)} \mathrm{d} t+\int_{0}^{T} \int_{\Omega_{1 \varepsilon}} A^{\varepsilon} \nabla u_{1 \varepsilon} \nabla v_{1} \mathrm{~d} x \mathrm{~d} t \\
\quad+\int_{0}^{T} \int_{\Omega_{2 \varepsilon}} A^{\varepsilon} \nabla u_{2 \varepsilon} \nabla v_{2} \mathrm{~d} x \mathrm{~d} t+\int_{0}^{T} \varepsilon^{\gamma} \int_{\Gamma^{\varepsilon}} h^{\varepsilon}\left(u_{1 \varepsilon}-u_{2 \varepsilon}\right)\left(v_{1}-v_{2}\right) \mathrm{d} \sigma_{x} \mathrm{~d} t \\
\quad=\int_{0}^{T} \int_{\Omega_{1 \varepsilon}} f_{1 \varepsilon} v_{1} \mathrm{~d} x \mathrm{~d} t+\int_{0}^{T}\left\langle g, P_{1}^{\varepsilon} v_{1}\right\rangle_{H^{-1}(\Omega), H_{0}^{1}(\Omega)}+\int_{0}^{T} \int_{\Omega_{2 \varepsilon}} f_{2 \varepsilon} v_{2} \mathrm{~d} x \mathrm{~d} t \\
\text { for every }\left(v_{1}, v_{2}\right) \in L^{2}\left(0, T ; V^{\varepsilon}\right) \times L^{2}\left(0, T ; H^{1}\left(\Omega_{2 \varepsilon}\right)\right), \\
u_{1 \varepsilon}(x, 0)=U_{1 \varepsilon}^{0} \text { in } \Omega_{1 \varepsilon} \text { and } u_{2 \varepsilon}(x, 0)=U_{2 \varepsilon}^{0} \text { in } \Omega_{2 \varepsilon},
\end{array}
\end{array}\right.
$$

where

$$
\begin{gathered}
W^{\varepsilon}:=\left\{v=\left(v_{1}, v_{2}\right) \in L^{2}\left(0, T ; V^{\varepsilon}\right) \times L^{2}\left(0, T ; H^{1}\left(\Omega_{2 \varepsilon}\right)\right)\right. \text { such that } \\
\left.v^{\prime} \in L^{2}\left(0, T ;\left(V^{\varepsilon}\right)^{\prime}\right) \times L^{2}\left(0, T ;\left(H^{1}\left(\Omega_{2 \varepsilon}\right)\right)^{\prime}\right)\right\}
\end{gathered}
$$

equipped with the norm

$$
\|v\|_{W^{\varepsilon}}=\left\|v_{1}\right\|_{L^{2}\left(0, T ; V^{\varepsilon}\right)}+\left\|v_{2}\right\|_{L^{2}\left(0, T ; H^{1}\left(\Omega_{2 \varepsilon}\right)\right)}+\left\|v_{1}^{\prime}\right\|_{L^{2}\left(0, T ;\left(V^{\varepsilon}\right)^{\prime}\right)}+\left\|v_{2}^{\prime}\right\|_{L^{2}\left(0, T ;\left(H^{1}\left(\Omega_{2 \varepsilon}\right)\right)^{\prime}\right)} .
$$

In [21] (see also [19]), the limit behaviour as $\varepsilon$ tends to zero of problem (2.14) has been described for $\gamma \leq 1$. When $-1<\gamma \leq 1$, which is the case studied in this paper, two different homogenized (limit) problems were obtained and are given in Theorem 2.5 below. To do so, let $\widehat{w}_{\lambda} \in H^{1}\left(Y_{1}\right)$ for any $\lambda \in \mathbb{R}^{n}$, be the solution of the problem

and $A^{0}$ the homogenized matrix given by

$$
\begin{cases}-\operatorname{div}\left(A \nabla \widehat{w}_{\lambda}\right)=0 & \text { in } Y_{1} \\ \left(A \nabla \widehat{w}_{\lambda}\right) \cdot n_{1}=0 & \text { in } \Gamma \\ \widehat{w}_{\lambda}-\lambda \cdot y & Y \text {-periodic } \\ \frac{1}{\left|Y_{1}\right|} \int_{Y_{1}}\left(\widehat{w}_{\lambda}-\lambda \cdot y\right) \mathrm{d} y=0\end{cases}
$$

$$
A^{0} \lambda:=m_{Y}\left(A \nabla \widehat{w}_{\lambda}\right)
$$

Theorem 2.5 [21]. Let $A^{\varepsilon}$ and $h^{\varepsilon}$ be defined by (2.9) and (2.11) respectively. Let $-1<\gamma \leq 1$ and $u_{\varepsilon}$ be the solution of problem (2.12). Moreover, suppose that

$$
\left\{\begin{array}{l}
g \in L^{2}\left(0, T ; H^{-1}(\Omega)\right) \\
\left(U_{1 \varepsilon}^{0}, U_{2 \varepsilon}^{0}\right) \in L^{2}\left(\Omega_{1 \varepsilon}\right) \times L^{2}\left(\Omega_{2 \varepsilon}\right) \\
\left(f_{1 \varepsilon}, f_{2 \varepsilon}\right) \in L^{2}\left(0, T ; L^{2}(\Omega)\right) \times L^{2}\left(0, T ; L^{2}(\Omega)\right)
\end{array}\right.
$$

and

$$
\begin{cases}\left(\widetilde{U_{1 \varepsilon}^{0}}, \widetilde{U_{2 \varepsilon}^{0}}\right) \rightarrow\left(\theta_{1} U_{1}^{0}, \theta_{2} U_{2}^{0}\right) & \text { weakly in } L^{2}(\Omega) \times L^{2}(\Omega) \\ \left(\widetilde{f_{1 \varepsilon}}, \widetilde{f_{2 \varepsilon}}\right) \rightarrow\left(\theta_{1} f_{1}, \theta_{2} f_{2}\right) & \text { weakly in } L^{2}\left(0, T ; L^{2}(\Omega)\right) \times L^{2}\left(0, T ; L^{2}(\Omega)\right)\end{cases}
$$


where $\theta_{i}(i=1,2)$ is given by $(2.2)$. Then, there exists a suitable extension operator

$$
P_{1}^{\varepsilon} \in \mathcal{L}\left(L^{2}\left(0, T ; V^{\varepsilon}\right) ; L^{2}\left(0, T ; H_{0}^{1}(\Omega)\right)\right) \cap \mathcal{L}\left(L^{2}\left(0, T ; L^{2}\left(\Omega_{1 \varepsilon}\right)\right) ; L^{2}\left(0, T ; L^{2}(\Omega)\right)\right)
$$

such that

$$
\begin{cases}\left(\text { i) } P_{1}^{\varepsilon} u_{1 \varepsilon} \rightarrow u_{1}\right. & \text { weakly in } L^{2}\left(0, T ; H_{0}^{1}(\Omega)\right), \\ \left(\text { ii } \widetilde{u_{1 \varepsilon}} \rightarrow \theta_{1} u_{1}\right. & \text { weakly }{ }^{*} \text { in } L^{\infty}\left(0, T ; L^{2}(\Omega)\right), \\ \left(\text { iii } \widetilde{u_{2 \varepsilon}} \rightarrow u_{2}\right. & \text { weakly } \text { in } L^{\infty}\left(0, T ; L^{2}(\Omega)\right),\end{cases}
$$

where $c$ is a constant independent of $\varepsilon$. In addition,

$$
\begin{cases}\text { (i) } A^{\varepsilon} \widetilde{\nabla u_{1 \varepsilon}}-A^{0} \nabla u_{1} & \text { weakly in } L^{2}\left(0, T ;\left[L^{2}(\Omega)\right]^{n}\right), \\ \text { (ii }) A^{\varepsilon} \widetilde{\nabla u_{2 \varepsilon}} \rightarrow 0 & \text { weakly in } L^{2}\left(0, T ;\left[L^{2}(\Omega)\right]^{n}\right),\end{cases}
$$

where $A^{0}$ is given by (2.16). Moreover, the limit functions $u_{1}$ and $u_{2}$ are described as follows:

- Case $-1<\gamma<1$. We have $u_{2}=\theta_{2} u_{1}$, where $\theta_{2}$ is given by $(2.2)$ and $u_{1} \in \mathcal{C}^{0}\left([0, T] ; L^{2}(\Omega)\right) \cap$ $L^{2}\left(0, T ; H_{0}^{1}(\Omega)\right)$, with $u_{1}^{\prime} \in L^{2}\left(0, T ; H^{-1}(\Omega)\right)$ is the unique solution of the homogenized problem

$$
\begin{cases}u_{1}^{\prime}-\operatorname{div}\left(A^{0} \nabla u_{1}\right)=\theta_{1} f_{1}+\theta_{2} f_{2}+g & \text { in } \Omega \times] 0, T[, \\ u_{1}=0 & \text { on } \partial \Omega \times] 0, T[, \\ u_{1}(0)=\theta_{1} U_{1}^{0}+\theta_{2} U_{2}^{0} & \text { in } \Omega .\end{cases}
$$

- Case $\gamma=1$. The couple $\left(u_{1}, u_{2}\right) \in \mathcal{C}^{0}\left([0, T] ; L^{2}(\Omega)\right) \cap L^{2}\left(0, T ; H_{0}^{1}(\Omega)\right) \times \mathcal{C}^{0}\left([0, T] ; L^{2}(\Omega)\right)$ with $\left(u_{1}^{\prime}, u_{2}^{\prime}\right) \in$ $L^{2}\left(0, T ; H^{-1}(\Omega)\right) \times L^{2}\left(0, T ; L^{2}(\Omega)\right)$ is the unique solution of the problem (a PDE coupled with an ODE)

$$
\begin{cases}\theta_{1} u_{1}^{\prime}-\operatorname{div}\left(A^{0} \nabla u_{1}\right)+c_{h}\left(\theta_{2} u_{1}-u_{2}\right)=\theta_{1} f_{1}+g & \text { in } \Omega \times] 0, T[, \\ u_{2}^{\prime}-c_{h}\left(\theta_{2} u_{1}-u_{2}\right)=\theta_{2} f_{2} & \text { in } \Omega \times] 0, T[, \\ u_{1}=0 & \text { on } \partial \Omega \times] 0, T[, \\ u_{1}(0)=U_{1}^{0}, u_{2}(0)=\theta_{2} U_{2}^{0} & \text { in } \Omega,\end{cases}
$$

where $c_{h}=\frac{1}{\left|Y_{2}\right|} \int_{\Gamma} h(y) \mathrm{d} \sigma_{y}$.

\section{Statement of the PROBlem AND MAin RESUlt}

Let us first introduce the corrector matrix for the parabolic problem (2.12), which is the same as that obtained by Donato et al. in [16] for perforated domains.

Let $\left(e_{j}\right)_{j=1, \ldots, n}$ be the canonical basis of $\mathbb{R}^{n}$. Set $\widehat{w}_{j}=\widehat{w}_{e_{j}}$, where $\widehat{w}_{j} \in H^{1}\left(Y_{1}\right)$ is the solution of problem (2.15) written for $\lambda=e_{j}, j=1, \ldots, n$.

The corrector matrix $C^{\varepsilon}=\left(C_{i j}^{\varepsilon}\right)_{1 \leq i, j \leq n}$ is defined by

$$
\left\{\begin{array}{l}
C_{i j}^{\varepsilon}(x)=\widetilde{C_{i j}}\left(\frac{x}{\varepsilon}\right) \quad \text { a.e. on } \Omega, \\
C_{i j}(y):=\frac{\partial \widehat{w}_{j}}{\partial y_{i}}(y), \quad i, j=1, \ldots, n \quad \text { a.e. on } Y_{1},
\end{array}\right.
$$

where $\sim$ denotes the zero extension to the whole of $Y$. Now, define $\widehat{w}_{j}^{\varepsilon}$ by

$$
\widehat{w}_{j}^{\varepsilon}(x):=x_{j}-\varepsilon\left(Q_{1}\left(\widehat{\chi}_{j}\right)(x / \varepsilon)\right), \quad \widehat{\chi}_{j}=y_{j}-\widehat{w}_{j}(y),
$$

where $Q_{1}$ is a suitable extension operator introduced in [9]. 
It follows that if $C_{j}^{\varepsilon}$ denotes the $j$ th column, then

$$
C_{j}^{\varepsilon}(x)=\nabla \widehat{w}_{j}^{\varepsilon}\left(\frac{x}{\varepsilon}\right), \quad j=1,2, \ldots, n
$$

and for $c$ independent of $\varepsilon$,

$$
\left\|C^{\varepsilon}\right\|_{\left[L^{2}\left(\Omega_{1 \varepsilon}\right)\right]^{n^{2}}} \leq c
$$

Observe also that a change of scale in (2.15) gives

$$
\int_{\Omega_{1 \varepsilon}} A^{\varepsilon} \nabla \widehat{w}_{j}^{\varepsilon} \nabla v \mathrm{~d} x=0, \quad \forall v \in H_{0}^{1}(\Omega)
$$

and the following convergences hold:

$$
\begin{cases}(i) \widehat{w}_{j}^{\varepsilon}(x) \rightarrow x_{j} & \text { weakly in } H^{1}(\Omega), \\ (i i) \widehat{w}_{j}^{\varepsilon}(x) \rightarrow x_{j} & \text { strongly in } L^{2}(\Omega), \\ (i i i) \nabla \widehat{w}_{j}^{\varepsilon}(x) \rightarrow e_{j} & \text { weakly in }\left[L^{2}(\Omega)\right]^{n} \\ (i v) \chi_{\Omega_{1 \varepsilon}} A^{\varepsilon} \nabla \widehat{w}_{j}^{\varepsilon} \rightarrow A^{0} e_{j} & \text { weakly in }\left[L^{2}(\Omega)\right]^{n}\end{cases}
$$

We now introduce some assumptions on the data, stronger than (2.18), depending on $\gamma$. These assumptions, as already seen in $[3,15]$, are necessary (see Sect. 5) to provide the convergence of the energy of problem (2.14) to that of the homogenized one. This convergence, observed in [3], plays an essential role in the proof of corrector results.

Concerning the data $f_{i \varepsilon}(i=1,2)$, we suppose that for $-1<\gamma \leq 1, f_{i \varepsilon}$ is the restriction of a function defined on the whole of $\Omega$ and

$$
\begin{cases}f_{i \varepsilon} \in L^{2}\left(0, T ; L^{2}(\Omega)\right), & i=1,2 \\ \left(f_{1 \varepsilon}, f_{2 \varepsilon}\right) \rightarrow\left(f_{1}, f_{2}\right) & \text { strongly in } L^{2}\left(0, T ; L^{2}(\Omega)\right) \times L^{2}\left(0, T ; L^{2}(\Omega)\right) .\end{cases}
$$

This will imply that (see also Rem. 2.2),

$$
\left(\chi_{\Omega_{1 \varepsilon}} f_{1 \varepsilon}, \chi_{\Omega_{2 \varepsilon}} f_{2 \varepsilon}\right) \rightarrow\left(\theta_{1} f_{1}, \theta_{2} f_{2}\right) \quad \text { weakly in } L^{2}\left(0, T ; L^{2}(\Omega)\right) \times L^{2}\left(0, T ; L^{2}(\Omega)\right) .
$$

Let us now focus on the assumptions for the initial conditions.

- If $-1<\gamma<1$, we suppose that for some $U^{0} \in L^{2}(\Omega)$,

$$
\widetilde{U_{1 \varepsilon}^{0}}+\widetilde{U_{2 \varepsilon}^{0}} \rightarrow U^{0} \quad \text { strongly in } L^{2}(\Omega)
$$

The lemma below clarifies this assumption.

Lemma 3.1. Let $U_{i \varepsilon}^{0} \in L^{2}\left(\Omega_{i \varepsilon}\right)(i=1,2)$ and $U^{0} \in L^{2}(\Omega)$. Then (3.9) holds if and only if

$$
\left\{\begin{array}{l}
(i) U_{i \varepsilon}^{0} \in L^{2}\left(\Omega_{i \varepsilon}\right) \\
(\text { ii }) \widetilde{U_{i \varepsilon}^{0}} \rightarrow \theta_{i} U^{0} \quad \text { weakly in } L^{2}(\Omega) \\
(\text { iii })\left\|U_{1 \varepsilon}^{0}\right\|_{L^{2}\left(\Omega_{1 \varepsilon}\right)}^{2}+\left\|U_{2 \varepsilon}^{0}\right\|_{L^{2}\left(\Omega_{2 \varepsilon}\right)}^{2} \rightarrow\left\|U^{0}\right\|_{L^{2}(\Omega)}^{2}
\end{array}\right.
$$

Proof. Observe that from (3.10)(ii), we obtain

$$
\widetilde{U_{1 \varepsilon}^{0}}+\widetilde{U_{2 \varepsilon}^{0}} \rightarrow \theta_{1} U^{0}+\theta_{2} U^{0}=U^{0} \quad \text { weakly in } L^{2}(\Omega)
$$


Now, since $\Omega_{1 \varepsilon}$ and $\Omega_{2 \varepsilon}$ are disjoint, from (3.10)(iii) we get

$$
\int_{\Omega}\left(\widetilde{U_{1 \varepsilon}^{0}}+\widetilde{U_{2 \varepsilon}^{0}}\right)^{2} \mathrm{~d} x=\int_{\Omega}\left(\widetilde{U_{1 \varepsilon}^{0}}\right)^{2} \mathrm{~d} x+\int_{\Omega}\left(\widetilde{U_{2 \varepsilon}^{0}}\right)^{2} \mathrm{~d} x \rightarrow \int_{\Omega}\left(U^{0}\right)^{2} \mathrm{~d} x
$$

which together with (3.11) gives (3.9).

Conversely, suppose that (3.9) is satisfied. For every $\varphi \in L^{2}(\Omega)$,

$$
\left.\int_{\Omega} \widetilde{U_{1 \varepsilon}^{0}} \varphi \mathrm{d} x=\int_{\Omega} \chi_{\Omega_{1 \varepsilon}} \widetilde{\left(U_{1 \varepsilon}^{0}\right.}+\widetilde{U_{2 \varepsilon}^{0}}\right) \varphi \mathrm{d} x \rightarrow \int_{\Omega} \theta_{1} U^{0} \varphi \mathrm{d} x
$$

Hence, $\widetilde{U_{1 \varepsilon}^{0}} \rightarrow \theta_{1} U^{0}$ weakly in $L^{2}(\Omega)$. Similarly, $\widetilde{U_{2 \varepsilon}^{0}} \rightarrow \theta_{2} U^{0}$ weakly in $L^{2}(\Omega)$. So we have (ii). Meanwhile, by the definition of $L^{2}$-norm and (3.12),

$$
\left\|U_{1 \varepsilon}^{0}\right\|_{L^{2}\left(\Omega_{1 \varepsilon}\right)}^{2}+\left\|U_{2 \varepsilon}^{0}\right\|_{L^{2}\left(\Omega_{2 \varepsilon}\right)}^{2}=\left\|\widetilde{U_{1 \varepsilon}^{0}}+\widetilde{U_{2 \varepsilon}^{0}}\right\|_{L^{2}(\Omega)}^{2} \rightarrow\left\|U^{0}\right\|_{L^{2}(\Omega)}^{2}
$$

This shows (iii) and ends the proof.

Remark 3.2. Observe that (3.10) holds, for instance, if $U_{i \varepsilon}^{0}$ is defined on the whole of $\Omega$ with $U_{i \varepsilon}^{0} \in L^{2}(\Omega)$ and there exists $U^{0} \in L^{2}(\Omega)$ such that for $i=1,2$,

$$
U_{i \varepsilon}^{0} \rightarrow U^{0} \quad \text { strongly in } L^{2}(\Omega) .
$$

Indeed, from Remark 2.2 we have, $\widetilde{U_{i \varepsilon}^{0} \mid \Omega_{i \varepsilon}} \rightarrow \theta_{i} U^{0}$ weakly in $L^{2}(\Omega)$. Also,

$$
\begin{aligned}
\int_{\Omega_{1 \varepsilon}}\left(U_{1 \varepsilon}^{0}\right)^{2} \mathrm{~d} x+\int_{\Omega_{2 \varepsilon}}\left(U_{2 \varepsilon}^{0}\right)^{2} \mathrm{~d} x & =\int_{\Omega} \chi_{\Omega_{1 \varepsilon}}\left(U_{1 \varepsilon}^{0}\right)^{2} \mathrm{~d} x+\int_{\Omega} \chi_{\Omega_{2 \varepsilon}}\left(U_{2 \varepsilon}^{0}\right)^{2} \mathrm{~d} x \\
& \rightarrow \int_{\Omega} \theta_{1}\left(U^{0}\right)^{2}+\int_{\Omega} \theta_{2}\left(U^{0}\right)^{2} \mathrm{~d} x=\left\|U^{0}\right\|_{L^{2}(\Omega)}^{2} .
\end{aligned}
$$

Remark 3.3. Let $-1<\gamma<1$ and suppose that (3.7) (from which (3.8) follows) and (3.9) are satisfied. It is clear (see also Rem. 2.2) that Theorem 2.5 applies, with $U_{1}^{0}=U_{2}^{0}=U^{0}$. Hence, the initial conditions in problem (2.21) reads

$$
u_{1}(0)=U^{0} .
$$

Let us now state our first corrector results which will be proved in Section 6 .

Theorem 3.4 (corrector results for the case $-1<\gamma<1$ ). Let $A^{\varepsilon}$ and $h^{\varepsilon}$ be defined by (2.9) and (2.11) respectively. Let $u_{\varepsilon}$ be the solution of problem (2.14). Suppose that (3.7) and (3.9) hold. Then, we have the following convergences:

$$
\left\{\begin{array}{l}
\text { (i) } \widetilde{u_{1 \varepsilon}}+\widetilde{u_{2 \varepsilon}} \rightarrow u_{1} \quad \text { in } \mathcal{C}^{0}\left([0, T] ; L^{2}(\Omega)\right), \\
\text { (ii) } \lim _{\varepsilon \rightarrow 0}\left\|\nabla u_{1 \varepsilon}-C^{\varepsilon} \nabla u_{1}\right\|_{L^{2}\left(0, T ;\left[L^{1}\left(\Omega_{1 \varepsilon}\right)\right]^{n}\right)}=0, \\
\text { (iii) } \lim _{\varepsilon \rightarrow 0}\left\|\nabla u_{2 \varepsilon}\right\|_{L^{2}\left(0, T ;\left[L^{2}\left(\Omega_{2 \varepsilon}\right)\right]^{n}\right)}=0,
\end{array}\right.
$$

where $u_{1}$ is the solution of the homogenized problem

$$
\begin{cases}u_{1}^{\prime}-\operatorname{div}\left(A^{0} \nabla u_{1}\right)=\theta_{1} f_{1}+\theta_{2} f_{2}+g & \text { in } \Omega \times] 0, T[, \\ u_{1}=0 & \text { on } \partial \Omega \times] 0, T[, \\ u_{1}(0)=U^{0} & \text { in } \Omega .\end{cases}
$$


Remark 3.5. From the proof of Proposition 5.3, it can be seen that (3.7) and (3.9) gives the necessary conditions for the convergence of the energy of problem (2.14) to that of the homogenized one when $-1<\gamma<1$.

- Consider now the case $\gamma=1$. We make the following assumptions on the initial conditions. For some $U_{i}^{0} \in L^{2}(\Omega)(i=1,2)$, suppose that

$$
\left\{\begin{array}{l}
(i) U_{i \varepsilon}^{0} \in L^{2}\left(\Omega_{i \varepsilon}\right) \\
\left(\text { ii) } \widetilde{U_{i \varepsilon}^{0}} \rightarrow \theta_{i} U_{i}^{0} \text { weakly in } L^{2}(\Omega),\right. \\
\left(\text { iii }\left\|U_{1 \varepsilon}^{0}\right\|_{L^{2}\left(\Omega_{1 \varepsilon}\right)}^{2}+\left\|U_{2 \varepsilon}^{0}\right\|_{L^{2}\left(\Omega_{2 \varepsilon}\right)}^{2} \rightarrow \theta_{1}\left\|U_{1}^{0}\right\|_{L^{2}(\Omega)}^{2}+\theta_{2}\left\|U_{2}^{0}\right\|_{L^{2}(\Omega)}^{2}\right.
\end{array}\right.
$$

Remark 3.6. Assumption (3.15) holds, for instance, if $U_{i \varepsilon}^{0}$ is defined on the whole of $\Omega$, with $U_{i \varepsilon}^{0} \in L^{2}(\Omega)$ and if for some $U_{i}^{0} \in L^{2}(\Omega)$, one has

$$
U_{i \varepsilon}^{0} \rightarrow U_{i}^{0} \quad \text { strongly in } L^{2}(\Omega), \quad \text { for } i=1,2 .
$$

Indeed, for every $\varphi \in L^{2}(\Omega)$,

$$
\int_{\Omega_{i \varepsilon}} U_{i \varepsilon}^{0} \varphi \mathrm{d} x=\int_{\Omega} \chi_{\Omega_{i \varepsilon}} U_{i \varepsilon}^{0} \varphi \mathrm{d} x \rightarrow \int_{\Omega} \theta_{i} U_{i}^{0} \varphi \mathrm{d} x, \quad \text { for } i=1,2 .
$$

Hence, $\widetilde{\left.U_{i \varepsilon}^{0}\right|_{\Omega_{i \varepsilon}}} \rightarrow \theta_{i} U_{i}^{0}$ weakly in $L^{2}(\Omega)$. On the other hand,

$$
\begin{aligned}
\left\|U_{1 \varepsilon}^{0}\right\|_{L^{2}\left(\Omega_{1 \varepsilon}\right)}^{2}+\left\|U_{2 \varepsilon}^{0}\right\|_{L^{2}\left(\Omega_{2 \varepsilon}\right)}^{2} & =\int_{\Omega} \chi_{\Omega_{1 \varepsilon}}\left(U_{1 \varepsilon}^{0}\right)^{2} \mathrm{~d} x+\int_{\Omega} \chi_{\Omega_{2 \varepsilon}}\left(U_{2 \varepsilon}^{0}\right)^{2} \mathrm{~d} x \\
& \rightarrow \int_{\Omega} \theta_{1}\left(U_{1}^{0}\right)^{2} \mathrm{~d} x+\int_{\Omega} \theta_{2}\left(U_{2}^{0}\right)^{2} \mathrm{~d} x=\theta_{1}\left\|U_{1}^{0}\right\|_{L^{2}(\Omega)}^{2}+\theta_{2}\left\|U_{2}^{0}\right\|_{L^{2}(\Omega)}^{2} .
\end{aligned}
$$

Remark 3.7. Observe also that if (3.15) holds with $U_{1}^{0}=U_{2}^{0}=U^{0}$ for some $U^{0} \in L^{2}(\Omega)$, then

$$
\widetilde{U_{1 \varepsilon}^{0}}+\widetilde{U_{2 \varepsilon}^{0}} \rightarrow U^{0} \quad \text { strongly in } L^{2}(\Omega),
$$

which is (3.9). This is because from (3.15)(ii) we obtain

$$
\widetilde{U_{1 \varepsilon}^{0}}+\widetilde{U_{2 \varepsilon}^{0}} \rightarrow \theta_{1} U^{0}+\theta_{2} U^{0}=U^{0} \quad \text { weakly in } L^{2}(\Omega) .
$$

Moreover, since the support of $\chi_{\Omega_{1 \varepsilon}}$ and $\chi_{\Omega_{2 \varepsilon}}$ are disjoint, we get the convergence of the norms from (iii), so that

$$
\left\|\widetilde{U_{1 \varepsilon}^{0}}+\widetilde{U_{2 \varepsilon}^{0}}\right\|_{L^{2}(\Omega)}^{2}=\left\|U_{1 \varepsilon}^{0}\right\|_{L^{2}\left(\Omega_{1 \varepsilon}\right)}^{2}+\left\|U_{2 \varepsilon}^{0}\right\|_{L^{2}\left(\Omega_{2 \varepsilon}\right)}^{2} \rightarrow\left\|U^{0}\right\|_{L^{2}(\Omega)}^{2}
$$

Hence, (3.15) is a more general condition than (3.9).

Remark 3.8. Let $\gamma=1$. Since (3.7) clearly implies (3.8), then under assumptions (3.7) and (3.15), Theorem 2.5 applies and the homogenized problem is still given by $(2.22)$.

We give in the following theorem the corrector results for the second case, which is proved in Section 6 .

Theorem 3.9 (corrector results for the case $\gamma=1$ ). Let $A^{\varepsilon}$ and $h^{\varepsilon}$ be defined by (2.9) and (2.11) respectively, and assume that $\Gamma$ is of class $C^{2}$. Let $u_{\varepsilon}$ be the solution of problem (2.14). Suppose that (3.7) and (3.15) hold. 
Then, we have the following convergences:

$$
\left\{\begin{array}{l}
\left(\text { i) } \lim _{\varepsilon \rightarrow 0}\left\|u_{1 \varepsilon}-u_{1}\right\|_{\mathcal{C}^{0}\left(0, T ; L^{2}\left(\Omega_{1 \varepsilon}\right)\right)}=0\right. \\
\text { (ii) } \lim _{\varepsilon \rightarrow 0}\left\|u_{2 \varepsilon}-\theta_{2}^{-1} u_{2}\right\|_{\mathcal{C}^{0}\left(0, T ; L^{2}\left(\Omega_{2 \varepsilon}\right)\right)}=0 \\
\text { (iii) } \lim _{\varepsilon \rightarrow 0}\left\|\nabla u_{1 \varepsilon}-C^{\varepsilon} \nabla u_{1}\right\|_{L^{2}\left(0, T ;\left[L^{1}\left(\Omega_{1 \varepsilon}\right)\right]^{n}\right)}=0 \\
\text { (iv) } \lim _{\varepsilon \rightarrow 0}\left\|\nabla u_{2 \varepsilon}\right\|_{L^{2}\left(0, T ;\left[L^{2}\left(\Omega_{2 \varepsilon}\right)\right]^{n}\right)}=0
\end{array}\right.
$$

where $\left(u_{1}, u_{2}\right)$ is the solution of the homogenized problem (2.22).

Remark 3.10. It can be seen in Section 5 that (3.7) and (3.15) are exactly what we need for the convergence of the energy in the case $\gamma=1$.

One of the main tools in proving the corrector results is a compactness result for $\widetilde{u_{1 \varepsilon}}+\widetilde{u_{2 \varepsilon}}$ in $\mathcal{C}^{0}\left([0, T] ; H^{-1}(\Omega)\right)$, which is shown in the next section (Thm. 4.8).

\section{A PRIORI ESTimates FOR $\left(u^{\varepsilon}\right)^{\prime}$ AND A COMPACTNESS RESUlt}

In this section, we first study the dual of $H_{\gamma}^{\varepsilon}$ and complete the a priori estimates stated in [21], under the assumptions of Theorem 2.5. We begin with the following results concerning the space $H_{\gamma}^{\varepsilon}$ and its dual.

Proposition 4.1. (i) There exists a constant $c>0$ such that

$$
\|v\|_{H_{\gamma}^{\varepsilon}}^{2} \leq c\left(1+\varepsilon^{\gamma-1}\right)\|v\|_{V^{\varepsilon} \times H^{1}\left(\Omega_{2 \varepsilon}\right)}^{2}, \quad \forall \gamma \in \mathbb{R} .
$$

(ii) If $\gamma \leq 1$, there exist two positive constants $c_{1}, c_{2}$ (independent of $\varepsilon$ ) such that

$$
c_{1}\|v\|_{V^{\varepsilon} \times H^{1}\left(\Omega_{2 \varepsilon}\right)}^{2} \leq\|v\|_{H_{\gamma}^{\varepsilon}}^{2} \leq c_{2}\left(1+\varepsilon^{\gamma-1}\right)\|v\|_{V^{\varepsilon} \times H^{1}\left(\Omega_{2 \varepsilon}\right)}^{2} .
$$

Proof. (i) Observe that by the definition of the norms in $H_{\gamma}^{\varepsilon}, V^{\varepsilon}$ and $H^{1}\left(\Omega_{2 \varepsilon}\right)$ and by Lemma 2.4, one has

$$
\begin{aligned}
\|v\|_{H_{\gamma}^{\varepsilon}}^{2} & =\left\|\nabla v_{1}\right\|_{L^{2}\left(\Omega_{1 \varepsilon}\right)}^{2}+\left\|\nabla v_{2}\right\|_{L^{2}\left(\Omega_{2 \varepsilon}\right)}^{2}+\varepsilon^{\gamma}\left\|v_{1}-v_{2}\right\|_{L^{2}\left(\Gamma^{\varepsilon}\right)}^{2} \\
& =\left\|v_{1}\right\|_{V^{\varepsilon}}^{2}+\left\|\nabla v_{2}\right\|_{L^{2}\left(\Omega_{2 \varepsilon}\right)}^{2}+\varepsilon^{\gamma}\left\|v_{1}-v_{2}\right\|_{L^{2}\left(\Gamma^{\varepsilon}\right)}^{2} \\
& \leq\left\|v_{1}\right\|_{V^{\varepsilon}}^{2}+\left\|v_{2}\right\|_{H^{1}\left(\Omega_{2 \varepsilon}\right)}^{2}+\varepsilon^{\gamma-1} \varepsilon\left\|v_{1}-v_{2}\right\|_{L^{2}\left(\Gamma^{\varepsilon}\right)}^{2} \\
& \leq\left\|v_{1}\right\|_{V^{\varepsilon}}^{2}+\left\|v_{2}\right\|_{H^{1}\left(\Omega_{2 \varepsilon}\right)}^{2}+\varepsilon^{\gamma-1}\|v\|_{H_{1}^{\varepsilon}}^{2} \\
& \leq c_{2}\left(1+\varepsilon^{\gamma-1}\right)\|v\|_{V^{\varepsilon} \times H^{1}\left(\Omega_{2 \varepsilon}\right)}^{2} .
\end{aligned}
$$

(ii) The right-hand side inequality follows from (i). Now, if $\gamma \leq 1$, then (2.6) implies that $\|v\|_{H_{1}^{\varepsilon}}^{2} \leq\|v\|_{H_{\gamma}^{\varepsilon}}^{2}$. Together with Lemma 2.4, we have $c_{1}\|v\|_{V^{\varepsilon} \times H^{1}\left(\Omega_{2 \varepsilon}\right)}^{2} \leq\|v\|_{H_{\gamma}^{\varepsilon}}^{2}$ with $c_{1}=C_{2}^{-2}$.

What can be said about the dual space $\left(H_{\gamma}^{\varepsilon}\right)^{\prime}$ with respect to $\left(V^{\varepsilon}\right)^{\prime} \times\left(H^{1}\left(\Omega_{2 \varepsilon}\right)\right)^{\prime}$ ? An answer is provided by the next proposition, where we use the notation $x^{\prime}(x)$ for the duality pairing between the dual space $E^{\prime}$ and a Banach space $E$.

Proposition 4.2. For $\gamma \leq 1$, we have

$$
v \in\left(V^{\varepsilon}\right)^{\prime} \times\left(H^{1}\left(\Omega_{2 \varepsilon}\right)\right)^{\prime} \quad \text { if and only if } \quad v \in\left(H_{\gamma}^{\varepsilon}\right)^{\prime}
$$

and there exist positive constants $k_{1}, k_{2}$ (independent of $\varepsilon$ ) such that

$$
k_{1}\|v\|_{\left(H_{\gamma}^{\varepsilon}\right)^{\prime}}^{2} \leq\|v\|_{\left(V^{\varepsilon}\right)^{\prime} \times\left(H^{1}\left(\Omega_{2 \varepsilon}\right)\right)^{\prime}}^{2} \leq k_{2}\left(1+\varepsilon^{\gamma-1}\right)\|v\|_{\left(H_{\gamma}^{\varepsilon}\right)^{\prime}}^{2} .
$$


Proof. Let $\gamma \leq 1$ and suppose $v \in\left(V^{\varepsilon}\right)^{\prime} \times\left(H^{1}\left(\Omega_{2 \varepsilon}\right)\right)^{\prime}$. We have from Proposition 4.1(ii) that

$$
\begin{aligned}
|v(u)| & \leq\|v\|_{\left(V^{\varepsilon}\right)^{\prime} \times\left(H^{1}\left(\Omega_{2 \varepsilon}\right)\right)^{\prime}}\|u\|_{V^{\varepsilon} \times H^{1}\left(\Omega_{2 \varepsilon}\right)} \\
& \leq \frac{1}{\sqrt{c_{1}}}\|v\|_{\left(V^{\varepsilon}\right)^{\prime} \times\left(H^{1}\left(\Omega_{2 \varepsilon}\right)\right)^{\prime}}\|u\|_{H_{\gamma}^{\varepsilon}}
\end{aligned}
$$

Hence,

$$
\sup _{u \neq 0} \frac{|v(u)|}{\|u\|_{H_{\gamma}^{\varepsilon}}} \leq \frac{1}{\sqrt{c_{1}}}\|v\|_{\left(V^{\varepsilon}\right)^{\prime} \times\left(H^{1}\left(\Omega_{2 \varepsilon}\right)\right)^{\prime}} .
$$

Therefore, $v \in\left(H_{\gamma}^{\varepsilon}\right)^{\prime}$ and we have the first inequality with $k_{1}=c_{1}$.

Now, suppose that $v \in\left(H_{\gamma}^{\varepsilon}\right)^{\prime}$. By using again Proposition 4.1, we get

$$
\begin{aligned}
|v(u)| & \leq\|v\|_{\left(H_{\gamma}^{\varepsilon}\right)^{\prime}}\|u\|_{H_{\gamma}^{\varepsilon}} \\
& \leq \sqrt{c_{2}}\|v\|_{\left(H_{\gamma}^{\varepsilon}\right)^{\prime}}\left(1+\varepsilon^{\frac{\gamma-1}{2}}\right)\|u\|_{V^{\varepsilon} \times H^{1}\left(\Omega_{2 \varepsilon}\right)} .
\end{aligned}
$$

This yields

$$
\sup _{u \neq 0} \frac{|v(u)|}{\|u\|_{V^{\varepsilon} \times H^{1}\left(\Omega_{2 \varepsilon}\right)}} \leq \sqrt{c_{2}}\left(1+\varepsilon^{\frac{\gamma-1}{2}}\right)\|v\|_{\left(H_{\gamma}^{\varepsilon}\right)^{\prime}} .
$$

Therefore, $v \in\left(V^{\varepsilon}\right)^{\prime} \times\left(H^{1}\left(\Omega_{2 \varepsilon}\right)\right)^{\prime}$ and we have the second inequality with $k_{2}=c_{2}$.

Remark 4.3. Proposition 4.2 implies that if $v=\left(v_{1}, v_{2}\right) \in\left(V^{\varepsilon}\right)^{\prime} \times\left(H^{1}\left(\Omega_{2 \varepsilon}\right)\right)^{\prime}$ and $u=\left(u_{1}, u_{2}\right) \in V^{\varepsilon} \times H^{1}\left(\Omega_{2 \varepsilon}\right)$, then $\langle v, u\rangle_{\left(H_{\gamma}^{\varepsilon}\right)^{\prime}, H_{\gamma}^{\varepsilon}}=\left\langle v_{1}, u_{1}\right\rangle_{\left(V^{\varepsilon}\right)^{\prime}, V^{\varepsilon}}+\left\langle v_{2}, u_{2}\right\rangle_{\left(H^{1}\left(\Omega_{2 \varepsilon}\right)\right)^{\prime}, H^{1}\left(\Omega_{2 \varepsilon}\right)}$.

Now, in order to show a compactness result for $\widetilde{u_{1 \varepsilon}}+\widetilde{u_{2 \varepsilon}}$, we need to estimate first the quantity

$$
\left\|\left(\widetilde{u_{1 \varepsilon}}+\widetilde{u_{2 \varepsilon}}\right)^{\prime}\right\|_{L^{2}\left(0, T ; H^{-1}(\Omega)\right)}
$$

To this aim, we prove the following lemma.

Lemma 4.4. Let

$$
v \in L^{2}\left(0, T ; V^{\varepsilon}\right)\left(r e s p . v \in L^{2}\left(0, T ; H^{1}\left(\Omega_{2 \varepsilon}\right)\right)\right)
$$

with

$$
v^{\prime} \in L^{2}\left(0, T ;\left(V^{\varepsilon}\right)^{\prime}\right)\left(r e s p . v^{\prime} \in L^{2}\left(0, T ;\left(H^{1}\left(\Omega_{2 \varepsilon}\right)\right)^{\prime}\right)\right) .
$$

Then

$$
(\widetilde{v})^{\prime} \in L^{2}\left(0, T ; H^{-1}(\Omega)\right)
$$

and for every $\psi \in L^{2}\left(0, T ; H_{0}^{1}(\Omega)\right)$,

$$
\begin{gathered}
\left\langle(\widetilde{v})^{\prime}, \psi\right\rangle_{L^{2}\left(0, T ; H^{-1}(\Omega)\right), L^{2}\left(0, T ; H_{0}^{1}(\Omega)\right)}=\left\langle v^{\prime},\left.\psi\right|_{\Omega_{1 \varepsilon}}\right\rangle_{L^{2}\left(0, T ;\left(V^{\varepsilon}\right)^{\prime}\right), L^{2}\left(0, T ; V^{\varepsilon}\right)} \\
\left(\operatorname{resp} .\left\langle(\widetilde{v})^{\prime}, \psi\right\rangle_{L^{2}\left(0, T ; H^{-1}(\Omega)\right), L^{2}\left(0, T ; H_{0}^{1}(\Omega)\right)}=\left\langle v^{\prime},\left.\psi\right|_{\Omega_{2 \varepsilon}}\right\rangle_{L^{2}\left(0, T ;\left(H^{1}\left(\Omega_{2 \varepsilon}\right)\right)^{\prime}\right), L^{2}\left(0, T ; H^{1}\left(\Omega_{2 \varepsilon}\right)\right)}\right) .
\end{gathered}
$$

Proof. Observe that for all $\psi \in \mathcal{D}((0, T) \times \Omega)$

$$
\begin{aligned}
\left\langle v^{\prime},\left.\psi\right|_{\Omega_{1 \varepsilon}}\right\rangle_{L^{2}\left(0, T ;\left(V^{\varepsilon}\right)^{\prime}\right), L^{2}\left(0, T ; V^{\varepsilon}\right)} & =\int_{0}^{T}\left\langle v^{\prime},\left.\psi\right|_{\Omega_{1 \varepsilon}}\right\rangle_{\left(V^{\varepsilon}\right)^{\prime}, V^{\varepsilon}} \mathrm{d} t=-\int_{0}^{T} \int_{\Omega_{1 \varepsilon}} v \psi^{\prime} \mathrm{d} x \mathrm{~d} t \\
& =-\int_{0}^{T} \int_{\Omega} \widetilde{v} \psi^{\prime} \mathrm{d} x \mathrm{~d} t=\left\langle(\widetilde{v})^{\prime}, \psi\right\rangle_{\mathcal{D}^{\prime}((0, T) \times \Omega), \mathcal{D}((0, T) \times \Omega)} .
\end{aligned}
$$


Hence, by a density argument

$$
\begin{aligned}
\left|\left\langle(\widetilde{v})^{\prime}, \psi\right\rangle_{\mathcal{D}^{\prime}((0, T) \times \Omega), \mathcal{D}((0, T) \times \Omega)}\right| & =\left|\left\langle v^{\prime},\left.\psi\right|_{\Omega_{1 \varepsilon}}\right\rangle_{L^{2}\left(0, T ;\left(V^{\varepsilon}\right)^{\prime}\right), L^{2}\left(0, T ; V^{\varepsilon}\right)}\right| \\
& \leq\left\|v^{\prime}\right\|_{L^{2}\left(0, T ;\left(V^{\varepsilon}\right)^{\prime}\right)}\|\psi\|_{L^{2}\left(0, T ; V^{\varepsilon}\right)} \leq\left\|v^{\prime}\right\|_{L^{2}\left(0, T ;\left(V^{\varepsilon}\right)^{\prime}\right)}\|\psi\|_{L^{2}\left(0, T ; H_{0}^{1}(\Omega)\right)},
\end{aligned}
$$

for every $\psi \in L^{2}\left(0, T ; H_{0}^{1}(\Omega)\right)$. In a similar manner, if

$$
v \in L^{2}\left(0, T ; H^{1}\left(\Omega_{2 \varepsilon}\right)\right) \text { and } v^{\prime} \in L^{2}\left(0, T ;\left(H^{1}\left(\Omega_{2 \varepsilon}\right)\right)^{\prime}\right)
$$

then for every $\psi \in L^{2}\left(0, T ; H_{0}^{1}(\Omega)\right)$,

$$
\left|\left\langle(\widetilde{v})^{\prime}, \psi\right\rangle_{\mathcal{D}^{\prime}((0, T) \times \Omega), \mathcal{D}((0, T) \times \Omega)}\right| \leq\left\|v^{\prime}\right\|_{L^{2}\left(0, T ;\left(H^{1}\left(\Omega_{2 \varepsilon}\right)\right)^{\prime}\right)}\|\psi\|_{L^{2}\left(0, T ; H_{0}^{1}(\Omega)\right)} .
$$

Therefore, in both cases,

$$
(\widetilde{v})^{\prime} \in L^{2}\left(0, T ; H^{-1}(\Omega)\right) .
$$

Let us also recall the following extension result proved by Cioranescu and Donato in [7] concerning the operator $P_{1}^{\varepsilon}$ from (2.13).

Lemma $4.5[7]$. There exists a linear continuous extension operator

$$
P_{1}^{\varepsilon} \in \mathcal{L}\left(L^{2}\left(0, T ; V^{\varepsilon}\right) ; L^{2}\left(0, T ; H_{0}^{1}(\Omega)\right)\right) \cap \mathcal{L}\left(L^{2}\left(0, T ; L^{2}\left(\Omega_{1 \varepsilon}\right)\right) ; L^{2}\left(0, T ; L^{2}(\Omega)\right)\right)
$$

such that for some positive constant $c$ independent of $\varepsilon$ and for any $\varphi \in L^{2}\left(0, T ; V^{\varepsilon}\right)$ with $\varphi^{\prime} \in L^{2}\left(0, T ; L^{2}\left(\Omega_{1 \varepsilon}\right)\right)$, we have

$$
\left\{\begin{array}{l}
\left.P_{1}^{\varepsilon} \varphi=\varphi \text { in } \Omega_{1 \varepsilon} \times\right] 0, T[, \\
\left.P_{1}^{\varepsilon} \varphi^{\prime}=\left(P_{1}^{\varepsilon} \varphi\right)^{\prime} \text { in } \Omega \times\right] 0, T[, \\
\left\|P_{1}^{\varepsilon} \varphi\right\|_{L^{2}\left(0, T ; L^{2}(\Omega)\right)} \leq c\|\varphi\|_{L^{2}\left(0, T ; L^{2}\left(\Omega_{1 \varepsilon}\right)\right)}, \\
\left\|P_{1}^{\varepsilon} \varphi^{\prime}\right\|_{L^{2}\left(0, T ; L^{2}(\Omega)\right)} \leq c\left\|\varphi^{\prime}\right\|_{L^{2}\left(0, T ; L^{2}\left(\Omega_{1 \varepsilon}\right)\right)}, \\
\left.\left\|P_{1}^{\varepsilon} \varphi(t)\right\|_{H_{0}^{1}(\Omega)} \leq c\|\nabla \varphi(t)\|_{L^{2}\left(\Omega_{1 \varepsilon}\right)}, \text { a.e. in }\right] 0, T[, \\
\left\|\nabla\left(P_{1}^{\varepsilon} \varphi\right)\right\|_{L^{2}\left(0, T ;\left[L^{2}(\Omega)\right]^{n}\right)} \leq c\|\nabla \varphi\|_{L^{2}\left(0, T ;\left[L^{2}\left(\Omega_{1 \varepsilon}\right)\right]^{n}\right)} .
\end{array}\right.
$$

We will use in the sequel the following result (used in the proof of Cor. 2.8 of [21]), which is an adaptation to the time-dependent case of a lemma given in [4].

Lemma 4.6 [21]. Suppose that $\left(v_{\varepsilon}\right)$ and $\left(v_{\varepsilon}\right)^{\prime}$ are bounded in $L^{2}\left(0, T ; H_{0}^{1}(\Omega)\right)$ and $L^{2}\left(0, T ; L^{2}(\Omega)\right)$ respectively, with $v_{\varepsilon} \rightarrow v$ strongly in $L^{2}\left(0, T ; L^{2}(\Omega)\right)$. Then,

$$
P_{1}^{\varepsilon}\left(\left.v_{\varepsilon}\right|_{\Omega_{1 \varepsilon}}\right) \rightarrow v \quad \text { weakly in } L^{2}\left(0, T ; H_{0}^{1}(\Omega)\right)
$$

Consider now the solution $\left(u_{1 \varepsilon}, u_{2 \varepsilon}\right)$ of problem (2.14). The next proposition deals with the norms $\left\|u_{1 \varepsilon}^{\prime}\right\|$ and $\left\|u_{2 \varepsilon}^{\prime}\right\|$ in spaces $L^{2}\left(0, T ;\left(V^{\varepsilon}\right)^{\prime}\right)$ and $L^{2}\left(0, T ;\left(H^{1}\left(\Omega_{2 \varepsilon}\right)\right)^{\prime}\right)$ respectively. In addition, it will provide a priori estimates for $\left\|\left(\widetilde{u_{1 \varepsilon}}+\widetilde{u_{2 \varepsilon}}\right)^{\prime}\right\|_{L^{2}\left(0, T ; H^{-1}(\Omega)\right)}$. 
Theorem 4.7. Under the assumptions of Theorem 2.5, if $\gamma \leq 1$, there exists positive constant c, independent of $\varepsilon$, such that

$$
\left\{\begin{array}{l}
(i)\left\|u_{1 \varepsilon}^{\prime}\right\|_{L^{2}\left(0, T ;\left(V^{\varepsilon}\right)^{\prime}\right)} \leq c\left(1+\varepsilon^{\frac{\gamma-1}{2}}\right), \\
(\text { ii })\left\|u_{2 \varepsilon}^{\prime}\right\|_{L^{2}\left(0, T ;\left(H^{1}\left(\Omega_{2 \varepsilon}\right)\right)^{\prime}\right)} \leq c\left(1+\varepsilon^{\frac{\gamma-1}{2}}\right), \\
(\text { iii })\left\|\left(\widetilde{u_{1 \varepsilon}}+\widetilde{u_{2 \varepsilon}}\right)^{\prime}\right\|_{L^{2}\left(0, T ; H^{-1}(\Omega)\right)} \leq c .
\end{array}\right.
$$

Proof. Using (2.8), the variational formulation (2.14) and the Hölder inequality, we deduce that

$$
\begin{aligned}
& \left|\left\langle u_{1 \varepsilon}^{\prime}, v_{1}\right\rangle_{L^{2}\left(0, T ;\left(V^{\varepsilon}\right)^{\prime}\right), L^{2}\left(0, T ; V^{\varepsilon}\right)}+\left\langle u_{2 \varepsilon}^{\prime}, v_{2}\right\rangle_{L^{2}\left(0, T ;\left(H^{1}\left(\Omega_{2 \varepsilon}\right)\right)^{\prime}\right), L^{2}\left(0, T ; H^{1}\left(\Omega_{2 \varepsilon}\right)\right)}\right| \\
& =\mid \int_{0}^{T} \int_{\Omega_{1 \varepsilon}} f_{1 \varepsilon} v_{1} \mathrm{~d} x \mathrm{~d} t+\int_{0}^{T}\left\langle g, P_{1}^{\varepsilon} v_{1}\right\rangle_{H^{-1}(\Omega), H_{0}^{1}(\Omega)} \mathrm{d} t+\int_{0}^{T} \int_{\Omega_{2 \varepsilon}} f_{2 \varepsilon} v_{2} \mathrm{~d} x \mathrm{~d} t \\
& -\int_{0}^{T} \int_{\Omega_{1 \varepsilon}} A^{\varepsilon} \nabla u_{1 \varepsilon} \nabla v_{1} \mathrm{~d} x \mathrm{~d} t-\int_{0}^{T} \int_{\Omega_{2 \varepsilon}} A^{\varepsilon} \nabla u_{2 \varepsilon} \nabla v_{2} \mathrm{~d} x \mathrm{~d} t \\
& -\varepsilon^{\gamma} \int_{0}^{T} \int_{\Gamma^{\varepsilon}} h^{\varepsilon}\left(u_{1 \varepsilon}-u_{2 \varepsilon}\right)\left(v_{1}-v_{2}\right) \mathrm{d} \sigma_{x} \mathrm{~d} t \\
& \leq\left\|\widetilde{f_{1 \varepsilon}}\right\|_{L^{2}\left(0, T ; L^{2}(\Omega)\right)}\left\|v_{1}\right\|_{L^{2}\left(0, T ; L^{2}\left(\Omega_{1 \varepsilon}\right)\right)}+\|g\|_{L^{2}\left(0, T ; H^{-1}(\Omega)\right)}\left\|P_{1}^{\varepsilon} v_{1}\right\|_{L^{2}\left(0, T ; H_{0}^{1}(\Omega)\right)} \\
& +\left\|\widetilde{f_{2 \varepsilon}}\right\|_{L^{2}\left(0, T ; L^{2}(\Omega)\right)}\left\|v_{2}\right\|_{L^{2}\left(0, T ; L^{2}\left(\Omega_{2 \varepsilon}\right)\right)}+\beta\left\|\widetilde{\nabla u_{1 \varepsilon}}\right\|_{L^{2}\left(0, T ; L^{2}(\Omega)\right)}\left\|\nabla v_{1}\right\|_{L^{2}\left(0, T ; L^{2}\left(\Omega_{1 \varepsilon}\right)\right)} \\
& +\beta\left\|\widetilde{\nabla u_{2 \varepsilon}}\right\|_{L^{2}\left(0, T ; L^{2}(\Omega)\right)}\left\|\nabla v_{2}\right\|_{L^{2}\left(0, T ; L^{2}\left(\Omega_{2 \varepsilon}\right)\right)} \\
& +\left\|h^{\varepsilon}\right\|_{L^{\infty}\left(\Gamma^{\varepsilon}\right)} \varepsilon^{\frac{\gamma}{2}}\left\|u_{1 \varepsilon}-u_{2 \varepsilon}\right\|_{L^{2}\left(0, T ; L^{2}\left(\Gamma^{\varepsilon}\right)\right)} \varepsilon^{\frac{\gamma}{2}}\left\|v_{1}-v_{2}\right\|_{L^{2}\left(0, T ; L^{2}\left(\Gamma^{\varepsilon}\right)\right)} .
\end{aligned}
$$

From (2.18), (2.19), Lemma 4.5, Remark 2.3 and Proposition 4.1(ii) it follows that

$$
\begin{aligned}
\mid\left\langle u_{1 \varepsilon}^{\prime}, v_{1}\right\rangle_{L^{2}\left(0, T ;\left(V^{\varepsilon}\right)^{\prime}\right)} & L^{2}\left(0, T ; V^{\varepsilon}\right) \\
& \leq\left\langle u_{2 \varepsilon}^{\prime}, v_{2}\right\rangle_{L^{2}\left(0, T ;\left(H^{1}\left(\Omega_{2 \varepsilon}\right)\right)^{\prime}\right), L^{2}\left(0, T ; H^{1}\left(\Omega_{2 \varepsilon}\right)\right)} \mid \\
& \leq c\left(\left\|v_{1}\right\|_{L^{2}\left(0, T ; V^{\varepsilon}\right)}+\left\|v_{2}\right\|_{L^{2}\left(0, T ; H^{1}\left(\Omega_{2 \varepsilon}\right)\right)}+\varepsilon^{\frac{\gamma}{2}}\left\|v_{1}-v_{2}\right\|_{L^{2}\left(0, T ; L^{2}\left(\Gamma^{\varepsilon}\right)\right)}\right) \\
& \leq c\left\|\left(v_{1}, v_{2}\right)\right\|_{L^{2}\left(0, T ; H_{\gamma}^{\varepsilon}\right)} .
\end{aligned}
$$

Therefore, together with Proposition 4.1(i), we have for every $v \in L^{2}\left(0, T ; H_{\gamma}^{\varepsilon}\right)$

$$
\begin{aligned}
\mid\left\langle u_{1 \varepsilon}^{\prime}, v_{1}\right\rangle_{L^{2}\left(0, T ;\left(V^{\varepsilon}\right)^{\prime}\right)}, L^{2}\left(0, T ; V^{\varepsilon}\right) & +\left\langle u_{2 \varepsilon}^{\prime}, v_{2}\right\rangle_{L^{2}\left(0, T ;\left(H^{1}\left(\Omega_{2 \varepsilon}\right)\right)^{\prime}\right), L^{2}\left(0, T ; H^{1}\left(\Omega_{2 \varepsilon}\right)\right)} \mid \\
& \leq c\left\|\left(v_{1}, v_{2}\right)\right\|_{L^{2}\left(0, T ; H_{\gamma}^{\varepsilon}\right)} \\
& \leq c\left(1+\varepsilon^{\frac{\gamma-1}{2}}\right)\left\|\left(v_{1}, v_{2}\right)\right\|_{L^{2}\left(0, T ; V^{\varepsilon}\right) \times L^{2}\left(0, T ; H^{1}\left(\Omega_{2 \varepsilon}\right)\right)}
\end{aligned}
$$

Taking $v_{2}=0$ in (4.3), we get

$$
\left\|u_{1 \varepsilon}^{\prime}\right\|_{L^{2}\left(0, T ;\left(V^{\varepsilon}\right)^{\prime}\right)}=\sup \frac{\left|\left\langle u_{1 \varepsilon}^{\prime}, v_{1}\right\rangle_{L^{2}\left(0, T ;\left(V^{\varepsilon}\right)^{\prime}\right), L^{2}\left(0, T ; V^{\varepsilon}\right)}\right|}{\left\|v_{1}\right\|_{L^{2}\left(0, T ; V^{\varepsilon}\right)}} \leq c\left(1+\varepsilon^{\frac{\gamma-1}{2}}\right) .
$$

Similarly, taking $v_{1}=0$ in (4.3), gives

$$
\left\|u_{2 \varepsilon}^{\prime}\right\|_{L^{2}\left(0, T ;\left(H^{1}\left(\Omega_{2 \varepsilon}\right)\right)^{\prime}\right)} \leq c\left(1+\varepsilon^{\frac{\gamma-1}{2}}\right) .
$$


It remains to prove (iii). To do that, take $v_{1}=v_{2}=\psi$ in (4.2), where $\psi \in L^{2}\left(0, T ; H_{0}^{1}(\Omega)\right)$. Then, together with Lemma 4.4, one has

$$
\begin{aligned}
& \left|\left\langle\left(\widetilde{u_{1 \varepsilon}}+\widetilde{u_{2 \varepsilon}}\right)^{\prime}, \psi\right\rangle_{L^{2}\left(0, T ; H^{-1}(\Omega)\right), L^{2}\left(0, T ; H_{0}^{1}(\Omega)\right)}\right| \\
& \quad=\left|\left\langle u_{1 \varepsilon}^{\prime},\left.\psi\right|_{\Omega_{1 \varepsilon}}\right\rangle_{L^{2}\left(0, T ;\left(V^{\varepsilon}\right)^{\prime}\right), L^{2}\left(0, T ; V^{\varepsilon}\right)}+\left\langle u_{2 \varepsilon}^{\prime},\left.\psi\right|_{\Omega_{2 \varepsilon}}\right\rangle_{L^{2}\left(0, T ;\left(H^{1}\left(\Omega_{2 \varepsilon}\right)\right)^{\prime}\right), L^{2}\left(0, T ; H^{1}\left(\Omega_{2 \varepsilon}\right)\right)}\right| \\
& \quad \leq c\|(\psi, \psi)\|_{L^{2}\left(0, T ; H_{\gamma}^{\varepsilon}\right)}=c\|(\psi, \psi)\|_{L^{2}\left(0, T ; V^{\varepsilon}\right) \times L^{2}\left(0, T ; H^{1}\left(\Omega_{2 \varepsilon}\right)\right)} \leq 2 c\|\psi\|_{L^{2}\left(0, T ; H_{0}^{1}(\Omega)\right)} .
\end{aligned}
$$

This concludes the proof.

We are now in the position to give the main result of this section stating the compactness of $\widetilde{u_{1 \varepsilon}}+\widetilde{u_{2 \varepsilon}}$ in $\mathcal{C}^{0}\left([0, T] ; H^{-1}(\Omega)\right)$.

Theorem 4.8. Let $\gamma \leq 1$. Then, under the assumptions of Theorem 2.5, the following strong convergence holds:

$$
\widetilde{u_{1 \varepsilon}}+\widetilde{u_{2 \varepsilon}} \rightarrow \theta_{1} u_{1}+u_{2} \quad \text { in } \quad \mathcal{C}^{0}\left([0, T] ; H^{-1}(\Omega)\right) .
$$

Moreover, if $\gamma=1$, one has separately that

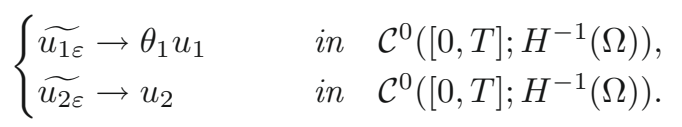

Proof. As a consequence of (2.19)(ii and iii) and (4.1)(iii),

$$
\begin{cases}\widetilde{u_{1 \varepsilon}}+\widetilde{u_{2 \varepsilon}} \rightarrow \theta_{1} u_{1}+u_{2} & \text { weakly* in } L^{\infty}\left(0, T ; L^{2}(\Omega)\right), \\ \left(\widetilde{u_{1 \varepsilon}}+\widetilde{u_{2 \varepsilon}}\right)^{\prime} & \text { is bounded in } L^{2}\left(0, T ; H^{-1}(\Omega)\right) .\end{cases}
$$

Hence, from classical compactness results (see [22]),

$$
\widetilde{u_{1 \varepsilon}}+\widetilde{u_{2 \varepsilon}} \text { is relatively compact in } \mathcal{C}^{0}\left([0, T] ; H^{-1}(\Omega)\right),
$$

whence, (4.4).

For the case $\gamma=1$, we apply to each of $\widetilde{u_{1 \varepsilon}}$ and $\widetilde{u_{2 \varepsilon}}$ the same arguments as that of the previous case. Observe that in this case from (4.1)(i and ii), the norms $\left\|u_{1 \varepsilon}^{\prime}\right\|_{L^{2}\left(0, T ;\left(V^{\varepsilon}\right)^{\prime}\right)}$ and $\left\|u_{2 \varepsilon}^{\prime}\right\|_{L^{2}\left(0, T ;\left(H^{1}\left(\Omega_{2 \varepsilon}\right)\right)^{\prime}\right)}$ are bounded. Then, the result is a consequence of Theorem 2.5 and Lemma 3.3 of [15] (which still holds by replacing $V^{\varepsilon}$ by $\left.H^{1}\left(\Omega_{2 \varepsilon}\right)\right)$.

Remark 4.9. We emphasize that for $\gamma<1$, the above theorem does not provide separately uniform estimates for $\widetilde{u_{1 \varepsilon}}$ and $\widetilde{u_{2 \varepsilon}}$ so that in this case, we only have the compactness (4.4) for the sum $\widetilde{u_{1 \varepsilon}}+\widetilde{u_{2 \varepsilon}}$. These results are sufficient for proving Propositions 6.5 and 6.8.

The relationship between the energies of problem (2.14) and those of limit problems (3.14) and (2.22) will play a crucial role when proving the corrector results. We discuss these in the following section.

\section{Asymptotic Behavior of the ENERGy}

In this section, we show that under the stronger assumptions on the data from Section 3, the energy of problem $(2.14)$ converges in $\mathcal{C}^{0}([0, T])$ to that of the homogenized one. By definition, the energy $d_{\varepsilon}$ associated to problem (2.14) is given by

$$
\begin{aligned}
d_{\varepsilon}(t)= & \frac{1}{2}\left\|u_{1 \varepsilon}(t)\right\|_{L^{2}\left(\Omega_{1 \varepsilon}\right)}^{2}+\frac{1}{2}\left\|u_{2 \varepsilon}(t)\right\|_{L^{2}\left(\Omega_{2 \varepsilon}\right)}^{2}+\int_{0}^{t} \int_{\Omega_{1 \varepsilon}} A^{\varepsilon} \nabla u_{1 \varepsilon} \nabla u_{1 \varepsilon} \mathrm{d} x \mathrm{~d} s \\
& +\int_{0}^{t} \int_{\Omega_{2 \varepsilon}} A^{\varepsilon} \nabla u_{2 \varepsilon} \nabla u_{2 \varepsilon} \mathrm{d} x \mathrm{~d} s+\varepsilon^{\gamma} \int_{0}^{t} \int_{\Gamma^{\varepsilon}} h^{\varepsilon}\left|u_{1 \varepsilon}-u_{2 \varepsilon}\right|^{2} \mathrm{~d} \sigma_{x} \mathrm{~d} s .
\end{aligned}
$$


Now, suppose $u_{\varepsilon}=\left(u_{1 \varepsilon}, u_{2 \varepsilon}\right)$ is the solution to problem (2.12). By taking $\left(u_{1 \varepsilon}, u_{2 \varepsilon}\right)$ as test function in (2.14) and integrating by parts, we get

$$
\begin{aligned}
\frac{1}{2}\left\|u_{1 \varepsilon}(t)\right\|_{L^{2}\left(\Omega_{1 \varepsilon}\right)}^{2}+\frac{1}{2} \| & u_{2 \varepsilon}(t) \|_{L^{2}\left(\Omega_{2 \varepsilon}\right)}^{2}+\int_{0}^{t} \int_{\Omega_{1 \varepsilon}} A^{\varepsilon} \nabla u_{1 \varepsilon} \nabla u_{1 \varepsilon} \mathrm{d} x \mathrm{~d} s \\
& +\int_{0}^{t} \int_{\Omega_{2 \varepsilon}} A^{\varepsilon} \nabla u_{2 \varepsilon} \nabla u_{2 \varepsilon} \mathrm{d} x \mathrm{~d} s+\varepsilon^{\gamma} \int_{0}^{t} \int_{\Gamma^{\varepsilon}} h^{\varepsilon}\left|u_{1 \varepsilon}-u_{2 \varepsilon}\right|^{2} \mathrm{~d} \sigma_{x} \mathrm{~d} s \\
& =\frac{1}{2}\left\|u_{1 \varepsilon}(0)\right\|_{L^{2}\left(\Omega_{1 \varepsilon}\right)}^{2}+\frac{1}{2}\left\|u_{2 \varepsilon}(0)\right\|_{L^{2}\left(\Omega_{2 \varepsilon}\right)}^{2}+\int_{0}^{t} \int_{\Omega_{1 \varepsilon}} f_{1 \varepsilon} u_{1 \varepsilon} \mathrm{d} x \mathrm{~d} s \\
& +\int_{0}^{t}\left\langle g, P_{1}^{\varepsilon} u_{1 \varepsilon}\right\rangle_{H^{-1}(\Omega), H_{0}^{1}(\Omega)} \mathrm{d} s+\int_{0}^{t} \int_{\Omega_{2 \varepsilon}} f_{2 \varepsilon} u_{2 \varepsilon} \mathrm{d} x \mathrm{~d} s .
\end{aligned}
$$

Hence, $d_{\varepsilon}(t)$ can be rewritten as

$$
\begin{aligned}
d_{\varepsilon}(t)= & \frac{1}{2}\left\|U_{1 \varepsilon}^{0}\right\|_{L^{2}\left(\Omega_{1 \varepsilon}\right)}^{2}+\frac{1}{2}\left\|U_{2 \varepsilon}^{0}\right\|_{L^{2}\left(\Omega_{2 \varepsilon}\right)}^{2}+\int_{0}^{t} \int_{\Omega_{1 \varepsilon}} f_{1 \varepsilon} u_{1 \varepsilon} \mathrm{d} x \mathrm{~d} s \\
& +\int_{0}^{t}\left\langle g, P_{1}^{\varepsilon} u_{1 \varepsilon}\right\rangle_{H^{-1}(\Omega), H_{0}^{1}(\Omega)} \mathrm{d} s+\int_{0}^{t} \int_{\Omega_{2 \varepsilon}} f_{2 \varepsilon} u_{2 \varepsilon} \mathrm{d} x \mathrm{~d} s .
\end{aligned}
$$

We now show that $\left\{d_{\varepsilon}\right\}$ is relatively compact in $\mathcal{C}^{0}([0, T])$.

Proposition 5.1. Under the hypotheses of Theorem 2.5, there exists a subsequence (still denoted by $\varepsilon$ ) and $d_{0} \in \mathcal{C}^{0}([0, T])$ such that $d_{\varepsilon} \rightarrow d_{0}$ in $\mathcal{C}^{0}([0, T])$.

Proof. From (5.3) and the Hölder inequality, we have

$$
\begin{aligned}
\left|d_{\varepsilon}(t)\right| \leq & \frac{1}{2}\left\|U_{1 \varepsilon}^{0}\right\|_{L^{2}\left(\Omega_{1 \varepsilon}\right)}^{2}+\frac{1}{2}\left\|U_{2 \varepsilon}^{0}\right\|_{L^{2}\left(\Omega_{2 \varepsilon}\right)}^{2}+\left\|\widetilde{f_{1 \varepsilon}}\right\|_{L^{2}\left(0, T ; L^{2}(\Omega)\right)}\left\|\widetilde{u_{1 \varepsilon}}\right\|_{L^{2}\left(0, T ; L^{2}(\Omega)\right)} \\
& +\left\|\widetilde{f_{2 \varepsilon}}\right\|_{L^{2}\left(0, T ; L^{2}(\Omega)\right)}\left\|\widetilde{u_{2 \varepsilon}}\right\|_{L^{2}\left(0, T ; L^{2}(\Omega)\right)}+\|g\|_{L^{2}\left(0, T ; H^{-1}(\Omega)\right)}\left\|P_{1}^{\varepsilon} u_{1 \varepsilon}\right\|_{L^{2}\left(0, T ; H_{0}^{1}(\Omega)\right)}
\end{aligned}
$$

so that in view of Theorem $2.5,\left\{d_{\varepsilon}\right\}$ is bounded in $L^{\infty}(0, T)$.

Now, for any $t \in[0, T]$ and $h>0$ small enough, one has

$$
\begin{aligned}
\left|d_{\varepsilon}(t+h)-d_{\varepsilon}(t)\right| \leq & h^{1 / 2}\left\|\widetilde{u_{1 \varepsilon}}\right\|_{L^{\infty}\left(0, T ; L^{2}(\Omega)\right)}\left\|\widetilde{f_{1 \varepsilon}}\right\|_{L^{2}\left(0, T ; L^{2}(\Omega)\right)}+h^{1 / 2}\left\|\widetilde{u_{2 \varepsilon}}\right\|_{L^{\infty}\left(0, T ; L^{2}(\Omega)\right)}\left\|\widetilde{f_{2 \varepsilon}}\right\|_{L^{2}\left(0, T ; L^{2}(\Omega)\right)} \\
& +\|g\|_{L^{2}\left([t, t+h] ; H^{-1}(\Omega)\right)}\left\|P_{1}^{\varepsilon} u_{1 \varepsilon}\right\|_{L^{2}\left(0, T ; H_{0}^{1}(\Omega)\right)} .
\end{aligned}
$$

Using again Theorem 2.5 and taking $h \rightarrow 0$, we get

$$
\left|d_{\varepsilon}(t+h)-d_{\varepsilon}(t)\right| \leq c\left(h^{1 / 2}+\|g\|_{L^{2}\left([t, t+h] ; H^{-1}(\Omega)\right)}\right) \rightarrow 0 \quad \text { uniformly in } \varepsilon .
$$

Hence, by Ascoli-Arzela's Theorem, $d_{\varepsilon} \rightarrow d_{0}$ for some $d_{0}$ in $\mathcal{C}^{0}([0, T])$.

We introduce now the energy associated with the homogenized problem for the two cases.

- For the case $-1<\gamma<1$, the energy associated with the limit problem (3.14), denoted by $d$ is defined by

$$
d(t)=\frac{1}{2}\left\|u_{1}(t)\right\|_{L^{2}(\Omega)}^{2}+\int_{0}^{t} \int_{\Omega} A^{0} \nabla u_{1} \nabla u_{1} \mathrm{~d} x \mathrm{~d} s .
$$


We can rewrite this expression by multiplying the first equation of the homogenized problem (3.14) by $u_{1}$ and integrating by parts. Using the same argument as above for proving (5.2) and taking into account the initial conditions of (3.14), we get

$$
\begin{aligned}
d(t) & =\frac{1}{2}\left\|u_{1}(0)\right\|_{L^{2}(\Omega)}^{2}+\int_{0}^{t} \int_{\Omega}\left(\theta_{1} f_{1}+\theta_{2} f_{2}\right) u_{1} \mathrm{~d} x \mathrm{~d} s+\int_{0}^{t}\left\langle g, u_{1}\right\rangle_{H^{-1}(\Omega), H_{0}^{1}(\Omega)} \mathrm{d} s \\
& =\frac{1}{2}\left\|U^{0}\right\|_{L^{2}(\Omega)}^{2}+\int_{0}^{t} \int_{\Omega}\left(\theta_{1} f_{1}+\theta_{2} f_{2}\right) u_{1} \mathrm{~d} x \mathrm{~d} s+\int_{0}^{t}\left\langle g, u_{1}\right\rangle_{H^{-1}(\Omega), H_{0}^{1}(\Omega)} \mathrm{d} s
\end{aligned}
$$

- For $\gamma=1$, the energy associated with the homogenized problem (2.22), denoted by $d_{1}$ is defined by

$$
\begin{aligned}
d_{1}(t)= & \frac{1}{2} \theta_{1}\left\|u_{1}(t)\right\|_{L^{2}(\Omega)}^{2}+\frac{1}{2} \theta_{2}^{-1}\left\|u_{2}(t)\right\|_{L^{2}(\Omega)}^{2}+\int_{0}^{t} \int_{\Omega} A^{0} \nabla u_{1} \nabla u_{1} \mathrm{~d} x \mathrm{~d} s \\
& +c_{h} \theta_{2}^{-1} \int_{0}^{t} \int_{\Omega}\left(\theta_{2} u_{1}-u_{2}\right)^{2} \mathrm{~d} x \mathrm{~d} s .
\end{aligned}
$$

Proposition 5.2. If $\gamma=1$,

$$
\begin{aligned}
d_{1}(t)= & \frac{1}{2} \theta_{1}\left\|U_{1}^{0}\right\|_{L^{2}(\Omega)}^{2}+\frac{1}{2} \theta_{2}\left\|U_{2}^{0}\right\|_{L^{2}(\Omega)}^{2}+\int_{0}^{t} \int_{\Omega} \theta_{1} f_{1} u_{1} \mathrm{~d} x \mathrm{~d} s \\
& +\int_{0}^{t}\left\langle g, u_{1}\right\rangle_{H^{-1}(\Omega), H_{0}^{1}(\Omega)} \mathrm{d} s+\int_{0}^{t} \int_{\Omega} f_{2} u_{2} \mathrm{~d} x \mathrm{~d} s .
\end{aligned}
$$

Proof. Taking $u_{1}$ in the first equation and $\theta_{2}^{-1} u_{2}$ in the second equation of the homogenized problem (2.22) and integrating by parts, we have

$$
\begin{gathered}
\int_{0}^{t}\left\langle\theta_{1} u_{1}^{\prime}, u_{1}\right\rangle_{L^{2}(\Omega), L^{2}(\Omega)} \mathrm{d} s+\int_{0}^{t}\left\langle u_{2}^{\prime}, \theta_{2}^{-1} u_{2}\right\rangle_{L^{2}(\Omega), L^{2}(\Omega)} \mathrm{d} s+\int_{0}^{t} \int_{\Omega} A^{0} \nabla u_{1} \nabla u_{1} \mathrm{~d} x \mathrm{~d} s \\
+c_{h} \int_{0}^{t} \int_{\Omega}\left(\theta_{2} u_{1}-u_{2}\right) u_{1} \mathrm{~d} x \mathrm{~d} s-c_{h} \int_{0}^{t} \int_{\Omega}\left(\theta_{2} u_{1}-u_{2}\right) \theta_{2}^{-1} u_{2} \mathrm{~d} x \mathrm{~d} s \\
\quad=\int_{0}^{t} \int_{\Omega} \theta_{1} f_{1} u_{1} \mathrm{~d} x \mathrm{~d} s+\int_{0}^{t}\left\langle g, u_{1}\right\rangle_{H^{-1}(\Omega), H_{0}^{1}(\Omega)} \mathrm{d} s+\int_{0}^{t} \int_{\Omega} \theta_{2} f_{2}\left(\theta_{2}^{-1} u_{2}\right) \mathrm{d} x \mathrm{~d} s .
\end{gathered}
$$

Now,

$$
\int_{0}^{t}\left\langle\theta_{1} u_{1}^{\prime}, u_{1}\right\rangle_{L^{2}(\Omega), L^{2}(\Omega)} \mathrm{d} s=\frac{1}{2} \theta_{1}\left\|u_{1}(t)\right\|_{L^{2}(\Omega)}^{2}-\frac{1}{2} \theta_{1}\left\|u_{1}(0)\right\|_{L^{2}(\Omega)}^{2}
$$

Similarly,

$$
\int_{0}^{t}\left\langle u_{2}^{\prime}, \theta_{2}^{-1} u_{2}\right\rangle_{L^{2}(\Omega), L^{2}(\Omega)} \mathrm{d} s=\frac{1}{2} \theta_{2}^{-1}\left\|u_{2}(t)\right\|_{L^{2}(\Omega)}^{2}-\frac{1}{2} \theta_{2}^{-1}\left\|u_{2}(0)\right\|_{L^{2}(\Omega)}^{2}
$$

Also,

$$
c_{h} \int_{0}^{t} \int_{\Omega}\left(\theta_{2} u_{1}-u_{2}\right) u_{1} \mathrm{~d} x \mathrm{~d} s-c_{h} \int_{0}^{t} \int_{\Omega}\left(\theta_{2} u_{1}-u_{2}\right) \theta_{2}^{-1} u_{2} \mathrm{~d} x \mathrm{~d} s=c_{h} \theta_{2}^{-1} \int_{0}^{t} \int_{\Omega}\left(\theta_{2} u_{1}-u_{2}\right)^{2} \mathrm{~d} x \mathrm{~d} s .
$$


Therefore,

$$
\begin{aligned}
d_{1}(t)= & \frac{1}{2} \theta_{1}\left\|u_{1}(0)\right\|_{L^{2}(\Omega)}^{2}+\frac{1}{2} \theta_{2}^{-1}\left\|u_{2}(0)\right\|_{L^{2}(\Omega)}^{2}+\int_{0}^{t} \int_{\Omega} \theta_{1} f_{1} u_{1} \mathrm{~d} x \mathrm{~d} s \\
& +\int_{0}^{t}\left\langle g, u_{1}\right\rangle_{H^{-1}(\Omega), H_{0}^{1}(\Omega)} \mathrm{d} s+\int_{0}^{t} \int_{\Omega} f_{2} u_{2} \mathrm{~d} x \mathrm{~d} s .
\end{aligned}
$$

Using the initial conditions in (2.22), we get (5.7) since

$$
\frac{1}{2} \theta_{2}^{-1}\left\|\theta_{2} U_{2}^{0}\right\|_{L^{2}(\Omega)}^{2}=\frac{1}{2} \theta_{2}\left\|U_{2}^{0}\right\|_{L^{2}(\Omega)}^{2} .
$$

In the following propositions, we prove that for both cases, the energy $d_{\varepsilon}$ converges to the respective energy of the associated homogenized problem.

Proposition 5.3 (convergence of energy for $-1<\gamma<1$ ). Let $A^{\varepsilon}$ and $h^{\varepsilon}$ be defined by (2.9) and (2.11) respectively. Suppose that (3.7) and (3.9) hold. If $\left(u_{1 \varepsilon}, u_{2 \varepsilon}\right)$ is the solution of problem (2.14) and $u_{1}$ is the solution of the homogenized problem (3.14) then

$$
d_{\varepsilon} \rightarrow d \quad \text { in } \quad \mathcal{C}^{0}([0, T])
$$

where $d_{\varepsilon}$ and $d$ are given by (5.3) and (5.5) respectively.

Proof. As noted in Remark 3.3, we can apply the homogenization results stated in Theorem 2.5. From (3.7) and (2.19)(ii and iii), we obtain

$$
\lim _{\varepsilon \rightarrow 0} \int_{0}^{t} \int_{\Omega_{1 \varepsilon}} f_{1 \varepsilon} u_{1 \varepsilon} \mathrm{d} x \mathrm{~d} s=\lim _{\varepsilon \rightarrow 0} \int_{0}^{t} \int_{\Omega} f_{1 \varepsilon} \widetilde{u_{1 \varepsilon}} \mathrm{d} x \mathrm{~d} s=\int_{0}^{t} \int_{\Omega} f_{1} \theta_{1} u_{1} \mathrm{~d} x \mathrm{~d} s
$$

and

$$
\lim _{\varepsilon \rightarrow 0} \int_{0}^{t} \int_{\Omega_{2 \varepsilon}} f_{2 \varepsilon} u_{2 \varepsilon} \mathrm{d} x \mathrm{~d} s=\lim _{\varepsilon \rightarrow 0} \int_{0}^{t} \int_{\Omega} f_{2 \varepsilon} \widetilde{u_{2 \varepsilon}} \mathrm{d} x \mathrm{~d} s=\int_{0}^{t} \int_{\Omega} f_{2} u_{2} \mathrm{~d} x \mathrm{~d} s .
$$

Also, from (2.19)(i),

$$
\lim _{\varepsilon \rightarrow 0} \int_{0}^{t}\left\langle g,\left.P_{1}^{\varepsilon} u_{1 \varepsilon}\right|_{\Omega_{1 \varepsilon}}\right\rangle_{H^{-1}(\Omega), H_{0}^{1}(\Omega)} \mathrm{d} s=\int_{0}^{t}\left\langle g, u_{1}\right\rangle_{H^{-1}(\Omega), H_{0}^{1}(\Omega)} \mathrm{d} s .
$$

Clearly, from assumptions (3.9) and Lemma 3.1 we have

$$
\frac{1}{2}\left\|U_{1 \varepsilon}^{0}\right\|_{L^{2}\left(\Omega_{1 \varepsilon}\right)}^{2}+\frac{1}{2}\left\|U_{2 \varepsilon}^{0}\right\|_{L^{2}\left(\Omega_{2 \varepsilon}\right)}^{2} \rightarrow \frac{1}{2}\left\|U^{0}\right\|_{L^{2}(\Omega)}^{2} .
$$

Since $u_{2}=\theta_{2} u_{1}$, combining the above convergences and using Proposition 5.1, the conclusion follows.

Proposition 5.4 (convergence of energy for $\gamma=1$ ). Let $A^{\varepsilon}$ and $h^{\varepsilon}$ be defined by (2.9) and (2.11) respectively. Suppose that (3.7) and (3.15) hold. If $\left(u_{1 \varepsilon}, u_{2 \varepsilon}\right)$ is the solution of problem (2.14) and $\left(u_{1}, u_{2}\right)$ is the solution of the homogenized problem (2.22), then

$$
d_{\varepsilon} \rightarrow d_{1} \quad \text { in } \mathcal{C}^{0}([0, T]),
$$

where $d_{\varepsilon}$ and $d_{1}$ are given by (5.3) and (5.7) respectively. 
Proof. Under assumptions (3.7) and (3.15), Theorem 2.5 applies (see also Rem. 3.8). By using (3.7) and (2.19)(i, ii and iii), we still have (5.8)-(5.10). Obviously, by (3.15)(iii),

$$
\frac{1}{2}\left\|U_{1 \varepsilon}^{0}\right\|_{L^{2}\left(\Omega_{1 \varepsilon}\right)}^{2}+\frac{1}{2}\left\|U_{2 \varepsilon}^{0}\right\|_{L^{2}\left(\Omega_{2 \varepsilon}\right)}^{2} \rightarrow \frac{1}{2} \theta_{1}\left\|U_{1}^{0}\right\|_{L^{2}(\Omega)}^{2}+\frac{1}{2} \theta_{2}\left\|U_{2}^{0}\right\|_{L^{2}(\Omega)}^{2} .
$$

Therefore, by Proposition 5.1 and the above results, $d_{\varepsilon} \rightarrow d_{1}$ in $\mathcal{C}^{0}([0, T])$.

\section{Proof of the CORRECTOR Results}

We prove in this section the corrector results stated in Theorems 3.4 and 3.9. The proofs are rather technical and are based on the results of Sections 4 and 5. We adapt to our cases techniques used by Donato in the elliptic case [12], Donato et al. in the hyperbolic case [18] and Donato and Nabil for the parabolic case in perforated domains [15].

We recall some technical lemmas, the first one being a classical density result.

Lemma 6.1. Let $v \in L^{2}\left(0, T ; H_{0}^{1}(\Omega)\right) \cap \mathcal{C}^{0}\left([0, T] ; L^{2}(\Omega)\right)$. Then for any $\delta>0$, there exists $\phi \in \mathcal{C}^{\infty}(0, T ; \mathcal{D}(\Omega))$ such that

$$
\left\{\begin{array}{l}
(i)\|v-\phi\|_{\mathcal{C}^{0}\left([0, T] ; L^{2}(\Omega)\right)} \leq \delta \\
(i i)\|\nabla v-\nabla \phi\|_{L^{2}\left(0, T ; L^{2}(\Omega)\right)} \leq \delta .
\end{array}\right.
$$

The next lemma proved by Donato and Nabil [15] overcomes the technical difficulty in passing to the limit in products with two weakly converging sequences when one of them is independent of $t$.

Lemma 6.2 [15]. Let $\left(h_{\varepsilon}\right) \subset L^{p}\left(0, T ; W_{0}^{1, q}(\Omega)\right)$ and $\left(g_{\varepsilon}\right) \subset L^{q^{\prime}}(\Omega)$ with $p, q \geq 1$ and $\frac{1}{q}+\frac{1}{q^{\prime}}=1$ be two sequences such that

$$
\begin{cases}h_{\varepsilon} \rightarrow h & \text { weakly in } L^{p}\left(0, T ; W_{0}^{1, q}(\Omega)\right), \\ g_{\varepsilon} \rightarrow g & \text { weakly in } L^{q^{\prime}}(\Omega) .\end{cases}
$$

Then

$$
h_{\varepsilon} g_{\varepsilon} \rightarrow h g \quad \text { weakly in } L^{p}\left(0, T ; L^{1}(\Omega)\right) .
$$

We also state the following results, which are straightforward extensions to the time-dependent case of the results proved in $[6,14]$ (see also [12]) respectively. The first one provides an inequality for weakly convergent sequences while the second one transforms integral on the boundary $\Gamma^{\varepsilon}$ into volume integrals on $\Omega_{2 \varepsilon}$.

Lemma 6.3 [14]. Let $\mathcal{O}$ be an open set on $\mathbb{R}^{n}$ and, for every $\varepsilon$ let $\mathcal{O}_{\varepsilon}$ be an open set such that $\mathcal{O}_{\varepsilon} \subset \mathcal{O}$. Suppose that $v_{\varepsilon} \subset L^{2}\left(0, T ; L^{2}\left(\mathcal{O}_{\varepsilon}\right)\right)$ and for some $v \in L^{2}\left(0, T ; L^{2}(\mathcal{O})\right)$ the following convergences hold:

$$
\left\{\begin{array}{l}
\chi_{\mathcal{O}_{\varepsilon}} \rightarrow \chi_{0} \quad \text { weakly* in } L^{\infty}(\mathcal{O}), \\
\widetilde{v_{\varepsilon}} \rightarrow \chi_{0} v \quad \text { weakly in } L^{2}\left(0, T ; L^{2}(\mathcal{O})\right) .
\end{array}\right.
$$

Then

$$
\liminf _{\varepsilon \rightarrow 0} \int_{0}^{T} \int_{\mathcal{O}_{\varepsilon}}\left|v_{\varepsilon}\right|^{2} \mathrm{~d} x \mathrm{~d} t \geq \int_{0}^{T} \int_{\mathcal{O}} \chi_{0}|v|^{2} \mathrm{~d} x \mathrm{~d} t
$$

Lemma 6.4 [6,12]. Suppose that $\Gamma$ is of class $C^{2}$. Let $g$ be a function in $L^{\infty}(\Gamma)$ and set $c_{g}=\frac{1}{\left|Y_{2}\right|} \int_{\Gamma} g(y) \mathrm{d} \sigma_{y}$. If for some positive constant $c$ (independent of $\varepsilon$ ) one has

$$
\left\|v_{\varepsilon}\right\|_{L^{2}\left(0, T ; W^{1,1}\left(\Omega_{2 \varepsilon}\right)\right)} \leq c
$$


then

$$
\liminf _{\varepsilon \rightarrow 0} \varepsilon \int_{0}^{T} \int_{\Gamma^{\varepsilon}} g(x / \varepsilon) v_{\varepsilon}(x, t) \mathrm{d} \sigma_{x} \mathrm{~d} t=\liminf _{\varepsilon \rightarrow 0} c_{g} \int_{0}^{T} \int_{\Omega_{2 \varepsilon}} v_{\varepsilon}(x, t) \mathrm{d} x \mathrm{~d} t .
$$

We are now in the position to prove the corrector results. We consider first the case $-1<\gamma<1$.

\subsection{Corrector results for the case $-1<\gamma<1$}

We prove first the following proposition which is needed in the proof of the corrector results.

Proposition 6.5. Let $\Phi$ be in $\mathcal{C}^{\infty}(0, T ; \mathcal{D}(\Omega))$ and set

$$
\begin{aligned}
\eta_{\varepsilon}(t)= & \frac{1}{2}\left\|\widetilde{u_{1 \varepsilon}}(t)+\widetilde{u_{2 \varepsilon}}(t)-\Phi(t)\right\|_{L^{2}(\Omega)}^{2}+\int_{0}^{t} \int_{\Omega_{2 \varepsilon}} A^{\varepsilon} \nabla u_{2 \varepsilon} \nabla u_{2 \varepsilon} \mathrm{d} x \mathrm{~d} s \\
& +\int_{0}^{t} \int_{\Omega_{1 \varepsilon}} A^{\varepsilon}\left(\nabla u_{1 \varepsilon}-C^{\varepsilon} \nabla \Phi\right)\left(\nabla u_{1 \varepsilon}-C^{\varepsilon} \nabla \Phi\right) \mathrm{d} x \mathrm{~d} s .
\end{aligned}
$$

Then, under the assumptions of Theorem 3.4,

$$
\limsup _{\varepsilon \rightarrow 0}\left\|\eta_{\varepsilon}\right\|_{\mathcal{C}^{0}([0, T])} \leq\|\eta\|_{\mathcal{C}^{0}([0, T])}
$$

where $\eta(t)$ is given by

$$
\eta(t)=\frac{1}{2}\left\|u_{1}(t)-\Phi(t)\right\|_{L^{2}(\Omega)}^{2}+\int_{0}^{t} \int_{\Omega} A^{0}\left(\nabla u_{1}-\nabla \Phi\right)\left(\nabla u_{1}-\nabla \Phi\right) \mathrm{d} x \mathrm{~d} s .
$$

Remark 6.6. The above proposition is a weaker result compared to what can be seen in the general literature (see for instance $[3,15]$ ), where for appropriate $\eta_{\varepsilon}$ and $\eta$ one has

$$
\eta_{\varepsilon} \rightarrow \eta \quad \text { in } \mathcal{C}^{0}([0, T])
$$

Nevertheless, (6.3) is enough to prove our main theorem. A similar situation occurs in the same geometrical framework for the hyperbolic case [18].

Proof. We begin by decomposing (6.2) into three terms

$$
\eta_{\varepsilon}(t)=\eta_{\varepsilon}^{1}(t)-\eta_{\varepsilon}^{2}(t)+\eta_{\varepsilon}^{3}(t)
$$

where

$$
\begin{aligned}
& \eta_{\varepsilon}^{1}(t)=\frac{1}{2}\|\Phi(t)\|_{L^{2}(\Omega)}^{2}+\int_{0}^{t} \int_{\Omega_{1 \varepsilon}} A^{\varepsilon} C^{\varepsilon} \nabla \Phi C^{\varepsilon} \nabla \Phi \mathrm{d} x \mathrm{~d} s, \\
& \eta_{\varepsilon}^{2}(t)=\int_{\Omega}\left(\widetilde{u_{1 \varepsilon}}+\widetilde{u_{2 \varepsilon}}\right) \Phi \mathrm{d} x+\int_{0}^{t} \int_{\Omega_{1 \varepsilon}} A^{\varepsilon} C^{\varepsilon} \nabla \Phi \nabla u_{1 \varepsilon} \mathrm{d} x \mathrm{~d} s+\int_{0}^{t} \int_{\Omega_{1 \varepsilon}} A^{\varepsilon} \nabla u_{1 \varepsilon} C^{\varepsilon} \nabla \Phi \mathrm{d} x \mathrm{~d} s, \\
& \eta_{\varepsilon}^{3}(t)=\frac{1}{2}\left\|\widetilde{u_{1 \varepsilon}}(t)+\widetilde{u_{2 \varepsilon}}(t)\right\|_{L^{2}(\Omega)}^{2}+\int_{0}^{t} \int_{\Omega_{1 \varepsilon}} A^{\varepsilon} \nabla u_{1 \varepsilon} \nabla u_{1 \varepsilon} \mathrm{d} x \mathrm{~d} s+\int_{0}^{t} \int_{\Omega_{2 \varepsilon}} A^{\varepsilon} \nabla u_{2 \varepsilon} \nabla u_{2 \varepsilon} \mathrm{d} x \mathrm{~d} s .
\end{aligned}
$$

Step 1. Let us study first the term $\eta_{\varepsilon}^{2}(t)$ which is the most complicated. Our aim is to show that:

$$
\begin{aligned}
\lim _{\varepsilon \rightarrow 0} \eta_{\varepsilon}^{2}(t) & =\int_{\Omega} u_{1} \Phi \mathrm{d} x+\int_{0}^{t} \int_{\Omega} A^{0} \nabla \Phi \nabla u_{1} \mathrm{~d} x \mathrm{~d} s+\int_{0}^{t} \int_{\Omega} A^{0} \nabla u_{1} \nabla \Phi \mathrm{d} x \mathrm{~d} s \\
& :=\eta^{2}(t) \quad \text { in } \mathcal{C}^{0}([0, T]) .
\end{aligned}
$$


To do that, we decompose it into three terms defined below, that will be treated separately

$$
\eta_{\varepsilon}^{2}(t)=\kappa_{\varepsilon}^{1}(t)+\kappa_{\varepsilon}^{2}(t)+\kappa_{\varepsilon}^{3}(t)
$$

where

$$
\begin{aligned}
& \kappa_{\varepsilon}^{1}(t)=\int_{\Omega}\left(\widetilde{u_{1 \varepsilon}}+\widetilde{u_{2 \varepsilon}}\right) \Phi \mathrm{d} x \\
& \kappa_{\varepsilon}^{2}(t)=\int_{0}^{t} \int_{\Omega_{1 \varepsilon}} A^{\varepsilon} C^{\varepsilon} \nabla \Phi \nabla u_{1 \varepsilon} \mathrm{d} x \mathrm{~d} s \\
& \kappa_{\varepsilon}^{3}(t)=\int_{0}^{t} \int_{\Omega_{1 \varepsilon}} A^{\varepsilon} \nabla u_{1 \varepsilon} C^{\varepsilon} \nabla \Phi \mathrm{d} x \mathrm{~d} s
\end{aligned}
$$

Step 1.1. For the first term $\kappa_{\varepsilon}^{1}(t)$, observe that

$$
\max _{t \in[0, T]}\left|\int_{\Omega}\left[\left(\widetilde{u_{1 \varepsilon}}+\widetilde{u_{2 \varepsilon}}\right) \Phi-u_{1} \Phi\right] \mathrm{d} x\right| \leq\left\|\widetilde{u_{1 \varepsilon}}+\widetilde{u_{2 \varepsilon}}-u_{1}\right\|_{\mathcal{C}^{0}\left([0, T] ; H^{-1}(\Omega)\right)}\|\Phi\|_{\mathcal{C}^{0}\left([0, T] ; H_{0}^{1}(\Omega)\right)} .
$$

Hence, from Theorem 4.8 (see (4.4)) and recalling that $u_{2}=\theta_{2} u_{1}$ for the case $-1<\gamma<1$, we have

$$
\kappa_{\varepsilon}^{1}(t) \rightarrow \int_{\Omega} u_{1} \Phi \mathrm{d} x \quad \text { in } \mathcal{C}^{0}([0, T]) .
$$

Step 1.2. We consider now $\kappa_{\varepsilon}^{2}(t)$ and we follow the proof of Proposition 5.3 of [15]. Note that using (3.5) with $v=u_{1 \varepsilon} \frac{\partial \Phi}{\partial x_{i}}$, we have

$$
\kappa_{\varepsilon}^{2}(t)=\sum_{i=1}^{n} \int_{0}^{t} \int_{\Omega_{1 \varepsilon}} A^{\varepsilon} \nabla \widehat{w}_{i}^{\varepsilon} \frac{\partial \Phi}{\partial x_{i}} \nabla u_{1 \varepsilon} \mathrm{d} x \mathrm{~d} s=-\sum_{i=1}^{n} \int_{0}^{t} \int_{\Omega} \chi_{\Omega_{1 \varepsilon}} A^{\varepsilon} \nabla \widehat{w}_{i}^{\varepsilon} \nabla\left(\frac{\partial \Phi}{\partial x_{i}}\right) P_{1}^{\varepsilon} u_{1 \varepsilon} \mathrm{d} x \mathrm{~d} s .
$$

By (2.19)(i), (3.6)(iv) and Lemma 6.2 with $h_{\varepsilon}=P_{1}^{\varepsilon} u_{1 \varepsilon}$ and $g_{\varepsilon}=\chi_{\Omega_{1 \varepsilon}} A^{\varepsilon} \nabla \widehat{w}_{i}^{\varepsilon}$, we have

$$
\begin{aligned}
\lim _{\varepsilon \rightarrow 0} \kappa_{\varepsilon}^{2}(t) & =-\sum_{i=1}^{n} \int_{0}^{t} \int_{\Omega} A^{0} e_{i} \nabla\left(\frac{\partial \Phi}{\partial x_{i}}\right) u_{1} \mathrm{~d} x \mathrm{~d} s \\
& =\int_{0}^{t} \int_{\Omega} A^{0} \nabla \Phi \nabla u_{1} \mathrm{~d} x \mathrm{~d} s \quad \forall t \in[0, T] .
\end{aligned}
$$

Now, observe that by (2.19)(ii) and from the properties of $\Phi, A^{\varepsilon}, C^{\varepsilon}$ (given by (2.8)(ii) and (3.4)), it follows that $\kappa_{\varepsilon}^{2}(t)$ is bounded in $H^{1}(0, T)$.

Hence, by (6.9) and the compactness of the injection $H^{1}(0, T) \subset \mathcal{C}^{0}([0, T])$, we conclude that

$$
\kappa_{\varepsilon}^{2} \rightarrow \kappa^{2} \quad \text { in } \mathcal{C}^{0}([0, T]), \quad \text { where } \quad \kappa^{2}(t)=\int_{0}^{t} \int_{\Omega} A^{0} \nabla \Phi \nabla u_{1} \mathrm{~d} x \mathrm{~d} s
$$

Step 1.3. In this step, we study the third and last term $\kappa_{\varepsilon}^{3}$, which is the most delicate. Our tasks are to identify the pointwise limit of $\kappa_{\varepsilon}^{3}(t)$ and to show its compactness in $\mathcal{C}^{0}([0, T])$, the former being the main difficulty. Let us show first that

$$
\kappa_{\varepsilon}^{3}(t) \rightarrow \int_{0}^{t} \int_{\Omega} A^{0} \nabla u_{1} \nabla \Phi \mathrm{d} x \mathrm{~d} s \quad \forall t \in[0, T]
$$


Observe that

$$
\begin{aligned}
\kappa_{\varepsilon}^{3}(t) & =\sum_{i=1}^{n} \int_{0}^{t} \int_{\Omega_{1 \varepsilon}} A^{\varepsilon} \nabla u_{1 \varepsilon} \nabla \widehat{w}_{i}^{\varepsilon} \frac{\partial \Phi}{\partial x_{i}} \mathrm{~d} x \mathrm{~d} s \\
& =\sum_{i=1}^{n}\left\{\int_{0}^{t} \int_{\Omega_{1 \varepsilon}} A^{\varepsilon} \nabla u_{1 \varepsilon} \nabla\left(\widehat{w}_{i}^{\varepsilon} \frac{\partial \Phi}{\partial x_{i}}\right) \mathrm{d} x \mathrm{~d} s-\int_{0}^{t} \int_{\Omega_{1 \varepsilon}} A^{\varepsilon} \nabla u_{1 \varepsilon} \nabla\left(\frac{\partial \Phi}{\partial x_{i}}\right) \widehat{w}_{i}^{\varepsilon} \mathrm{d} x \mathrm{~d} s\right\} .
\end{aligned}
$$

Using the variational formulation (2.14) with $\left(\widehat{w}_{i}^{\varepsilon} \frac{\partial \Phi}{\partial x_{i}}, x_{i} \frac{\partial \Phi}{\partial x_{i}}\right)$ as test function and integrating by parts gives

$$
\begin{aligned}
\kappa_{\varepsilon}^{3}(t)= & \sum_{i=1}^{n}\left\{\int_{0}^{t} \int_{\Omega} \widetilde{f_{1 \varepsilon}} \widehat{w}_{i}^{\varepsilon} \frac{\partial \Phi}{\partial x_{i}} \mathrm{~d} x \mathrm{~d} s+\int_{0}^{t} \int_{\Omega} \widetilde{f_{2 \varepsilon}} x_{i} \frac{\partial \Phi}{\partial x_{i}} \mathrm{~d} x \mathrm{~d} s\right. \\
& +\int_{0}^{t}\left\langle g,\left.P_{1}^{\varepsilon}\left(\widehat{w}_{i}^{\varepsilon} \frac{\partial \Phi}{\partial x_{i}}\right)\right|_{\Omega_{1 \varepsilon}}\right\rangle_{H^{-1}(\Omega), H_{0}^{1}(\Omega)} \mathrm{d} s-\int_{0}^{t} \int_{\Omega} A^{\varepsilon} \widetilde{\nabla u_{2 \varepsilon}} \nabla\left(x_{i} \frac{\partial \Phi}{\partial x_{i}}\right) \mathrm{d} x \mathrm{~d} s \\
& -\int_{0}^{t}\left\langle u_{1 \varepsilon}^{\prime}, \widehat{w}_{i}^{\varepsilon} \frac{\partial \Phi}{\partial x_{i}}\right\rangle_{\left(V^{\varepsilon}\right)^{\prime}, V^{\varepsilon}} \mathrm{d} s-\int_{0}^{t}\left\langle u_{2 \varepsilon}^{\prime}, x_{i} \frac{\partial \Phi}{\partial x_{i}}\right\rangle_{\left(H^{1}\left(\Omega_{2 \varepsilon}\right)\right)^{\prime}, H^{1}\left(\Omega_{2 \varepsilon}\right)} \mathrm{d} s \\
& \left.-\varepsilon^{\gamma} \int_{0}^{t} \int_{\Gamma^{\varepsilon}} h^{\varepsilon}\left(u_{1 \varepsilon}-u_{2 \varepsilon}\right)\left(\widehat{w}_{i}^{\varepsilon} \frac{\partial \Phi}{\partial x_{i}}-x_{i} \frac{\partial \Phi}{\partial x_{i}}\right) \mathrm{d} \sigma_{x} \mathrm{~d} s-\int_{0}^{t} \int_{\Omega} A^{\varepsilon} \widetilde{\nabla u_{1 \varepsilon}} \nabla\left(\frac{\partial \Phi}{\partial x_{i}}\right) \widehat{w}_{i}^{\varepsilon} \mathrm{d} x \mathrm{~d} s\right\}
\end{aligned}
$$

Now, we evaluate the limit of (6.11) term by term. By using (3.8), (3.6)(ii) and Lemma 4.6, we have

$$
\begin{array}{r}
\sum_{i=1}^{n} \lim _{\varepsilon \rightarrow 0}\left\{\int_{0}^{t} \int_{\Omega} \widetilde{f_{1 \varepsilon}} \widehat{w}_{i}^{\varepsilon} \frac{\partial \Phi}{\partial x_{i}} \mathrm{~d} x \mathrm{~d} s+\int_{0}^{t} \int_{\Omega} \widetilde{f_{2 \varepsilon}} x_{i} \frac{\partial \Phi}{\partial x_{i}} \mathrm{~d} x \mathrm{~d} s+\int_{0}^{t}\left\langle g,\left.P_{1}^{\varepsilon}\left(\widehat{w}_{i}^{\varepsilon} \frac{\partial \Phi}{\partial x_{i}}\right)\right|_{\Omega_{1 \varepsilon}}\right\rangle_{H^{-1}(\Omega), H_{0}^{1}(\Omega)} \mathrm{d} s\right\} \\
=\sum_{i=1}^{n}\left\{\int_{0}^{t} \int_{\Omega}\left(\theta_{1} f_{1}+\theta_{2} f_{2}\right) x_{i} \frac{\partial \Phi}{\partial x_{i}} \mathrm{~d} x \mathrm{~d} s+\int_{0}^{t}\left\langle g, x_{i} \frac{\partial \Phi}{\partial x_{i}}\right\rangle_{H^{-1}(\Omega), H_{0}^{1}(\Omega)} \mathrm{d} s\right\}
\end{array}
$$

for every $t \in[0, T]$. On the other hand, from (2.20)(ii),

$$
\sum_{i=1}^{n} \lim _{\varepsilon \rightarrow 0} \int_{0}^{t} \int_{\Omega} A^{\varepsilon} \widetilde{\nabla u_{2 \varepsilon}} \nabla\left(x_{i} \frac{\partial \Phi}{\partial x_{i}}\right) \mathrm{d} x \mathrm{~d} s=0
$$

Meanwhile, by (2.20)(i) and (3.6)(ii),

$$
\sum_{i=1}^{n} \lim _{\varepsilon \rightarrow 0} \int_{0}^{t} \int_{\Omega} A^{\varepsilon} \widetilde{\nabla u_{1 \varepsilon}} \nabla\left(\frac{\partial \Phi}{\partial x_{i}}\right) \widehat{w}_{i}^{\varepsilon} \mathrm{d} x \mathrm{~d} s=\sum_{i=1}^{n} \int_{0}^{t} \int_{\Omega} A^{0} \nabla u_{1} \nabla\left(\frac{\partial \Phi}{\partial x_{i}}\right) x_{i} \mathrm{~d} x \mathrm{~d} s .
$$

Now, for the boundary term, observe that by (2.19)(iv), (3.2) and a change of scales,

$$
\begin{aligned}
\sum_{i=1}^{n} \varepsilon^{\gamma} \int_{0}^{t} \int_{\Gamma^{\varepsilon}} h^{\varepsilon}\left(u_{1 \varepsilon}-u_{2 \varepsilon}\right)\left(\widehat{w}_{i}^{\varepsilon}-x_{i}\right) \frac{\partial \Phi}{\partial x_{i}} \mathrm{~d} \sigma_{x} \mathrm{~d} s & \leq \sum_{i=1}^{n} \varepsilon^{\gamma}\|h\|_{L^{\infty}(\Gamma)}\left\|u_{1 \varepsilon}-u_{2 \varepsilon}\right\|_{L^{2}\left(0, T ; L^{2}\left(\Gamma^{\varepsilon}\right)\right)}\left\|\varepsilon \widehat{\chi}_{i}^{\varepsilon}\left(\frac{x}{\varepsilon}\right)\right\|_{L^{2}\left(\Gamma^{\varepsilon}\right)} \\
& \leq c \varepsilon^{\gamma+1} \varepsilon^{-\gamma / 2} \varepsilon^{-1 / 2}=c \varepsilon^{(\gamma+1) / 2} \rightarrow 0
\end{aligned}
$$

as $\varepsilon \rightarrow 0$, since $\gamma>-1$ and $\left\|\widehat{\chi}_{i}^{\varepsilon}\left(\frac{x}{\varepsilon}\right)\right\|_{L^{2}\left(\Gamma^{\varepsilon}\right)} \leq \varepsilon^{-1 / 2}$. 
It remains to show that $\forall t \in[0, T]$,

$$
\begin{aligned}
\sum_{i=1}^{n} \lim _{\varepsilon \rightarrow 0}\left\{\int _ { 0 } ^ { t } \left\langleu_{1 \varepsilon}^{\prime},\right.\right. & \left.\left.\widehat{w}_{i}^{\varepsilon} \frac{\partial \Phi}{\partial x_{i}}\right\rangle_{\left(V^{\varepsilon}\right)^{\prime}, V^{\varepsilon}} \mathrm{d} s+\int_{0}^{t}\left\langle u_{2 \varepsilon}^{\prime}, x_{i} \frac{\partial \Phi}{\partial x_{i}}\right\rangle_{\left(H^{1}\left(\Omega_{2 \varepsilon}\right)\right)^{\prime}, H^{1}\left(\Omega_{2 \varepsilon}\right)} \mathrm{d} s\right\} \\
& =\sum_{i=1}^{n} \int_{0}^{t}\left\langle\theta_{1} u_{1}^{\prime}+u_{2}^{\prime}, x_{i} \frac{\partial \Phi}{\partial x_{i}}\right\rangle_{H^{-1}(\Omega), H_{0}^{1}(\Omega)} \mathrm{d} s \\
& =\sum_{i=1}^{n} \int_{0}^{t}\left\langle u_{1}^{\prime}, x_{i} \frac{\partial \Phi}{\partial x_{i}}\right\rangle_{H^{-1}(\Omega), H_{0}^{1}(\Omega)} \mathrm{d} s .
\end{aligned}
$$

To prove (6.16), we rewrite

$$
\begin{aligned}
& \int_{0}^{t}\left\langle u_{1 \varepsilon}^{\prime}, \widehat{w}_{i}^{\varepsilon} \frac{\partial \Phi}{\partial x_{i}}\right\rangle_{\left(V^{\varepsilon}\right)^{\prime}, V^{\varepsilon}} \mathrm{d} s+\int_{0}^{t}\left\langle u_{2 \varepsilon}^{\prime}, x_{i} \frac{\partial \Phi}{\partial x_{i}}\right\rangle_{\left(H^{1}\left(\Omega_{2 \varepsilon}\right)\right)^{\prime}, H^{1}\left(\Omega_{2 \varepsilon}\right)} \mathrm{d} s \\
&=\int_{0}^{t}\left\langle u_{1 \varepsilon}^{\prime}, \widehat{w}_{i}^{\varepsilon} \frac{\partial \Phi}{\partial x_{i}}\right\rangle_{\left(V^{\varepsilon}\right)^{\prime}, V^{\varepsilon}} \mathrm{d} s+\int_{0}^{t}\left\langle u_{2 \varepsilon}^{\prime}, \widehat{w}_{i}^{\varepsilon} \frac{\partial \Phi}{\partial x_{i}}\right\rangle_{\left(H^{1}\left(\Omega_{2 \varepsilon}\right)\right)^{\prime}, H^{1}\left(\Omega_{2 \varepsilon}\right)} \mathrm{d} s \\
&+\int_{0}^{t}\left\langle u_{2 \varepsilon}^{\prime},\left(x_{i}-\widehat{w}_{i}^{\varepsilon}\right) \frac{\partial \Phi}{\partial x_{i}}\right\rangle_{\left(H^{1}\left(\Omega_{2 \varepsilon}\right)\right)^{\prime}, H^{1}\left(\Omega_{2 \varepsilon}\right)} \mathrm{d} s \\
&= \int_{0}^{t}\left\langle\left(\widetilde{u_{1 \varepsilon}}+\widetilde{u_{2 \varepsilon}}\right)^{\prime}, \widehat{w}_{i}^{\varepsilon} \frac{\partial \Phi}{\partial x_{i}}\right\rangle_{H^{-1}(\Omega), H_{0}^{1}(\Omega)} \mathrm{d} s \\
&+\int_{0}^{t}\left\langle u_{2 \varepsilon}^{\prime},\left(x_{i}-\widehat{w}_{i}^{\varepsilon}\right) \frac{\partial \Phi}{\partial x_{i}}\right\rangle_{\left(H^{1}\left(\Omega_{2 \varepsilon}\right)\right)^{\prime}, H^{1}\left(\Omega_{2 \varepsilon}\right)} \mathrm{d} s,
\end{aligned}
$$

where we used Lemma 4.4 for the last equality.

Using (3.2), we have

$$
\begin{aligned}
\int_{0}^{t}\left\langle u_{2 \varepsilon}^{\prime},\left(x_{i}-\widehat{w}_{i}^{\varepsilon}\right) \frac{\partial \Phi}{\partial x_{i}}\right\rangle_{\left(H^{1}\left(\Omega_{2 \varepsilon}\right)\right)^{\prime}, H^{1}\left(\Omega_{2 \varepsilon}\right)} \mathrm{d} s \\
=-\varepsilon \int_{0}^{t} \int_{\Omega} \widetilde{u_{2 \varepsilon}} \widehat{\chi}_{i}^{\varepsilon}\left(\frac{x}{\varepsilon}\right) \frac{\partial \Phi^{\prime}}{\partial x_{i}} \mathrm{~d} x \mathrm{~d} s+\varepsilon \int_{\Omega} \widetilde{u_{2 \varepsilon}}(t) \widehat{\chi}_{i}^{\varepsilon}\left(\frac{x}{\varepsilon}\right) \frac{\partial \Phi}{\partial x_{i}}(t) \mathrm{d} x-\varepsilon \int_{\Omega} \widetilde{U_{2 \varepsilon}^{0}} \widehat{\chi}_{i}^{\varepsilon}\left(\frac{x}{\varepsilon}\right) \frac{\partial \Phi}{\partial x_{i}}(0) \mathrm{d} x .
\end{aligned}
$$

By (2.19)(iii), (3.10)(ii) of Lemma 3.1 and the Hölder inequality,

$$
\sum_{i=1}^{n} \int_{0}^{t}\left\langle u_{2 \varepsilon}^{\prime},\left(x_{i}-\widehat{w}_{i}^{\varepsilon}\right) \frac{\partial \Phi}{\partial x_{i}}\right\rangle_{\left(H^{1}\left(\Omega_{2 \varepsilon}\right)\right)^{\prime}, H^{1}\left(\Omega_{2 \varepsilon}\right)} \mathrm{d} s \leq c \varepsilon \rightarrow 0 \quad \text { as } \varepsilon \rightarrow 0 .
$$

For the first term of (6.17), note that for all $t \in[0, T]$,

$$
\begin{aligned}
\int_{0}^{t}\left\langle\left(\widetilde{u_{1 \varepsilon}}+\widetilde{u_{2 \varepsilon}}\right)^{\prime}, \widehat{w}_{i}^{\varepsilon} \frac{\partial \Phi}{\partial x_{i}}\right\rangle_{H^{-1}(\Omega), H_{0}^{1}(\Omega)} \mathrm{d} s \\
\quad=-\int_{0}^{t} \int_{\Omega}\left(\widetilde{u_{1 \varepsilon}}+\widetilde{u_{2 \varepsilon}}\right) \widehat{w}_{i}^{\varepsilon} \frac{\partial \Phi^{\prime}}{\partial x_{i}} \mathrm{~d} x \mathrm{~d} s+\int_{\Omega}\left(\widetilde{u_{1 \varepsilon}}+\widetilde{u_{2 \varepsilon}}\right)(t) \widehat{w}_{i}^{\varepsilon} \frac{\partial \Phi}{\partial x_{i}}(t) \mathrm{d} x-\int_{\Omega}\left(\widetilde{U_{1 \varepsilon}^{0}}+\widetilde{U_{2 \varepsilon}^{0}}\right) \widehat{w}_{i}^{\varepsilon} \frac{\partial \Phi}{\partial x_{i}}(0) \mathrm{d} x
\end{aligned}
$$


where we pass to the limit term by term. Using the fact that $u_{2}=\theta_{2} u_{1}$ and by (2.19)(ii and iii) and (3.6)(ii), we get

$$
\begin{aligned}
\sum_{i=1}^{n} \lim _{\varepsilon \rightarrow 0} \int_{0}^{t} \int_{\Omega}\left(\widetilde{u_{1 \varepsilon}}+\widetilde{u_{2 \varepsilon}}\right) \widehat{w}_{i}^{\varepsilon} \frac{\partial \Phi^{\prime}}{\partial x_{i}} \mathrm{~d} x \mathrm{~d} s & =\sum_{i=1}^{n} \int_{0}^{t} \int_{\Omega}\left(\theta_{1} u_{1}+u_{2}\right) x_{i} \frac{\partial \Phi^{\prime}}{\partial x_{i}} \mathrm{~d} x \mathrm{~d} s \\
& =\sum_{i=1}^{n} \int_{0}^{t} \int_{\Omega} u_{1} x_{i} \frac{\partial \Phi^{\prime}}{\partial x_{i}} \mathrm{~d} x \mathrm{~d} s \quad \forall t \in[0, T] .
\end{aligned}
$$

On the other hand, by (3.10)(ii), (3.6)(ii) and in view of the initial conditions in (3.14),

$$
\begin{aligned}
\sum_{i=1}^{n} \lim _{\varepsilon \rightarrow 0} \int_{\Omega}\left(\widetilde{U_{1 \varepsilon}^{0}}+\widetilde{U_{2 \varepsilon}^{0}}\right) \widehat{w}_{i}^{\varepsilon} \frac{\partial \Phi}{\partial x_{i}}(0) \mathrm{d} x & =\sum_{i=1}^{n} \int_{\Omega}\left(\theta_{1} U^{0}+\theta_{2} U^{0}\right) x_{i} \frac{\partial \Phi}{\partial x_{i}}(0) \mathrm{d} x \\
& =\sum_{i=1}^{n} \int_{\Omega} u_{1}(0) x_{i} \frac{\partial \Phi}{\partial x_{i}}(0) \mathrm{d} x
\end{aligned}
$$

Lastly, the compactness result in Theorem 4.8 and (3.6)(ii and iii) imply that

$$
\begin{aligned}
\sum_{i=1}^{n} \lim _{\varepsilon \rightarrow 0} \int_{\Omega}\left(\widetilde{u_{1 \varepsilon}}+\widetilde{u_{2 \varepsilon}}\right)(t) \widehat{w_{i}^{\varepsilon}} \frac{\partial \Phi}{\partial x_{i}}(t) \mathrm{d} x & =\sum_{i=1}^{n} \lim _{\varepsilon \rightarrow 0}\left\langle\left(\widetilde{u_{1 \varepsilon}}+\widetilde{u_{2 \varepsilon}}\right)(t), \widehat{w_{i}^{\varepsilon}} \frac{\partial \Phi}{\partial x_{i}}(t)\right\rangle_{H^{-1}(\Omega), H_{0}^{1}(\Omega)} \\
& =\sum_{i=1}^{n}\left\langle\left(\theta_{1} u_{1}+u_{2}\right)(t), x_{i} \frac{\partial \Phi}{\partial x_{i}}(t)\right\rangle_{H^{-1}(\Omega), H_{0}^{1}(\Omega)} \\
& =\sum_{i=1}^{n} \int_{\Omega} u_{1}(t) x_{i} \frac{\partial \Phi}{\partial x_{i}}(t) \mathrm{d} x \quad \forall t \in[0, T] .
\end{aligned}
$$

Combining (6.19), (6.20) and (6.21) together with (6.18), we get (6.16).

Using convergences (6.12)-(6.16) in (6.11) we get

$$
\begin{aligned}
\lim _{\varepsilon \rightarrow 0} \kappa_{\varepsilon}^{3}(t)= & \sum_{i=1}^{n}\left\{\int_{0}^{t} \int_{\Omega}\left(\theta_{1} f_{1}+\theta_{2} f_{2}\right) x_{i} \frac{\partial \Phi}{\partial x_{i}} \mathrm{~d} x \mathrm{~d} s+\int_{0}^{t}\left\langle g, x_{i} \frac{\partial \Phi}{\partial x_{i}}\right\rangle_{H^{-1}(\Omega), H_{0}^{1}(\Omega)} \mathrm{d} s\right. & \\
& \left.-\int_{0}^{t}\left\langle u_{1}^{\prime}, x_{i} \frac{\partial \Phi}{\partial x_{i}}\right\rangle_{H^{-1}(\Omega), H_{0}^{1}(\Omega)} \mathrm{d} s-\int_{0}^{t} \int_{\Omega} A^{0} \nabla u_{1} \nabla\left(\frac{\partial \Phi}{\partial x_{i}}\right) x_{i} \mathrm{~d} x \mathrm{~d} s\right\} & \forall t \in[0, T] .
\end{aligned}
$$

On the other hand, observe that by taking $x_{i} \frac{\partial \Phi}{\partial x_{i}}$ as test function in the homogenized problem (3.14) and integrating by parts, we have

$$
\begin{aligned}
\sum_{i=1}^{n}\left\{\int_{0}^{t}\left\langle u_{1}^{\prime}, x_{i} \frac{\partial \Phi}{\partial x_{i}}\right\rangle_{H^{-1}(\Omega), H_{0}^{1}(\Omega)} \mathrm{d} s+\int_{0}^{t} \int_{\Omega} A^{0} \nabla u_{1} \nabla\left(x_{i} \frac{\partial \Phi}{\partial x_{i}}\right) \mathrm{d} x \mathrm{~d} s\right\} \\
=\sum_{i=1}^{n}\left\{\int_{0}^{t} \int_{\Omega}\left(\theta_{1} f_{1}+\theta_{2} f_{2}\right) x_{i} \frac{\partial \Phi}{\partial x_{i}} \mathrm{~d} x \mathrm{~d} s+\int_{0}^{t}\left\langle g, x_{i} \frac{\partial \Phi}{\partial x_{i}}\right\rangle_{H^{-1}(\Omega), H_{0}^{1}(\Omega)} \mathrm{d} s\right\} .
\end{aligned}
$$


Therefore, combining (6.22) and (6.23), we can deduce that

$$
\begin{aligned}
\lim _{\varepsilon \rightarrow 0} \kappa_{\varepsilon}^{3}(t) & =\sum_{i=1}^{n}\left\{\int_{0}^{t} \int_{\Omega} A^{0} \nabla u_{1} \nabla\left(x_{i} \frac{\partial \Phi}{\partial x_{i}}\right) \mathrm{d} x \mathrm{~d} s-\int_{0}^{t} \int_{\Omega} A^{0} \nabla u_{1} \nabla\left(\frac{\partial \Phi}{\partial x_{i}}\right) x_{i} \mathrm{~d} x \mathrm{~d} s\right\} \\
& =\sum_{i=1}^{n} \int_{0}^{t} \int_{\Omega} A^{0} \nabla u_{1} \nabla x_{i}\left(\frac{\partial \Phi}{\partial x_{i}}\right) \mathrm{d} x \mathrm{~d} s \\
& =\int_{0}^{t} \int_{\Omega} A^{0} \nabla u_{1} \nabla \Phi \mathrm{d} x \mathrm{~d} s \quad \forall t \in[0, T] .
\end{aligned}
$$

The Ascoli-Arzela Theorem shows that the above convergence is actually in $\mathcal{C}^{0}([0, T])$. Indeed, by $(2.20)(\mathrm{i})$, (3.4), our assumption on $\Phi$ and the Hölder inequality,

$$
\left|\kappa_{\varepsilon}^{3}(t)\right| \leq\left\|A^{\varepsilon} \widetilde{\nabla u_{1 \varepsilon}}\right\|_{L^{2}\left(0, T ;\left[L^{2}(\Omega)\right]^{n}\right)}\left\|C^{\varepsilon}\right\|_{\left[L^{2}(\Omega)\right]^{n^{2}}}\|\nabla \Phi\|_{L^{\infty}\left(0, T ;\left[L^{2}(\Omega)\right]^{n}\right)} \leq c,
$$

where $c$ is independent of $t$. Moreover, for any $h>0$ small enough,

$$
\begin{aligned}
\left|\kappa_{\varepsilon}^{3}(t+h)-\kappa_{\varepsilon}^{3}(t)\right| & \leq\left\|A^{\varepsilon} \widetilde{\nabla u_{1 \varepsilon}}\right\|_{L^{2}\left(0, T ;\left[L^{2}(\Omega)\right]^{n}\right)}\left\|C^{\varepsilon}\right\|_{\left[L^{2}(\Omega)\right]^{n^{2}}} h^{1 / 2}\|\nabla \Phi\|_{L^{\infty}\left(0, T ;\left[L^{2}(\Omega)\right]^{n}\right)} \\
& \leq c h^{1 / 2} \rightarrow 0, \quad \text { as } h \rightarrow 0, \text { uniformly in } \varepsilon .
\end{aligned}
$$

Hence,

$$
\kappa_{\varepsilon}^{3} \rightarrow \kappa^{3} \quad \text { in } \mathcal{C}^{0}([0, T]), \quad \text { where } \quad \kappa^{3}(t)=\int_{0}^{t} \int_{\Omega} A^{0} \nabla u_{1} \nabla \Phi \mathrm{d} x \mathrm{~d} s .
$$

Then, (6.6) follows from (6.7) and convergences (6.8), (6.10) and (6.24).

\section{Step 2. Since}

from (2.10) it follows that

$$
\left\|u_{1 \varepsilon}(t)\right\|_{L^{2}\left(\Omega_{1 \varepsilon}\right)}^{2}+\left\|u_{2 \varepsilon}(t)\right\|_{L^{2}\left(\Omega_{2 \varepsilon}\right)}^{2}=\left\|\widetilde{u_{1 \varepsilon}}(t)+\widetilde{u_{2 \varepsilon}}(t)\right\|_{L^{2}(\Omega)}^{2},
$$

$$
\eta_{\varepsilon}^{3}(t) \leq d_{\varepsilon}(t) \quad \forall t \in[0, T]
$$

where $d_{\varepsilon}(t)$ is the energy associated with problem (2.14) given by (5.1). This yields

$$
0 \leq \eta_{\varepsilon}(t)=\eta_{\varepsilon}^{3}(t)+\eta_{\varepsilon}^{1}(t)-\eta_{\varepsilon}^{2}(t) \leq d_{\varepsilon}(t)+\eta_{\varepsilon}^{1}(t)-\eta_{\varepsilon}^{2}(t), \quad \forall t \in[0, T] .
$$

On the other hand, it is known (see for instance, [16]) that

$$
\eta_{\varepsilon}^{1} \rightarrow \eta^{1} \quad \text { in } \mathcal{C}^{0}([0, T])
$$

where

Hence, by Proposition 5.3, (6.26) and (6.6), we have

$$
\eta^{1}(t)=\frac{1}{2}\|\Phi(t)\|_{L^{2}(\Omega)}^{2}+\int_{0}^{t} \int_{\Omega} A^{0} \nabla \Phi \nabla \Phi \mathrm{d} x \mathrm{~d} s .
$$

$$
d_{\varepsilon}+\eta_{\varepsilon}^{1}-\eta_{\varepsilon}^{2} \rightarrow d+\eta^{1}-\eta^{2}=\eta \quad \text { in } \mathcal{C}^{0}([0, T]),
$$

where $\eta(t)$ is given by (6.4) and $d$ by (5.4). This, together with (6.25), implies that

$$
\limsup _{\varepsilon \rightarrow 0}\left\|\eta_{\varepsilon}\right\|_{\mathcal{C}^{0}([0, T])} \leq \lim _{\varepsilon \rightarrow 0}\left\|d_{\varepsilon}+\eta_{\varepsilon}^{1}-\eta_{\varepsilon}^{2}\right\|_{\mathcal{C}^{0}([0, T])}=\|\eta\|_{\mathcal{C}^{0}([0, T])},
$$

which is (6.3) and the proof of Proposition 6.5 is complete. 
Remark 6.7. The non-symmetry of $A^{\varepsilon}$ makes $\eta_{\varepsilon}^{2}(t)$ different from its counterpart studied in [18] for the hyperbolic case, giving rise to the third term $\kappa_{\varepsilon}^{3}(t)$. This term is complicated to treat as already seen in the study of the heat equation in perforated domains studied in [15]. Here, we are able to conclude thanks to Lemma 4.4 and the compactness result proved in Theorem 4.8.

Proof of Theorem 3.4. Let $\delta>0$ be fixed and $\Phi \in \mathcal{C}^{\infty}(0, T ; \mathcal{D}(\Omega))$ be the corresponding function satisfying Lemma 6.1 and associated to the solution $u_{1}$ of (3.14). First, observe that by (2.8)(ii), Lemma 6.1 and Proposition 6.5,

$$
\begin{aligned}
\limsup _{\varepsilon \rightarrow 0}\left\|\eta_{\varepsilon}\right\|_{\mathcal{C}^{0}([0, T])} & \leq\|\eta\|_{\mathcal{C}^{0}([0, T])} \\
& \leq \frac{1}{2}\left\|u_{1}-\Phi\right\|_{\mathcal{C}^{0}\left([0, T] ; L^{2}(\Omega)\right)}^{2}+\int_{0}^{T} \int_{\Omega} A^{0}\left(\nabla u_{1}-\nabla \Phi\right)\left(\nabla u_{1}-\nabla \Phi\right) \mathrm{d} x \mathrm{~d} t \\
& \leq\left(\frac{1}{2}+\left\|A^{0}\right\|_{\left[L^{\infty}(\Omega)\right]^{n^{2}}}\right) \delta^{2}
\end{aligned}
$$

where $\eta_{\varepsilon}(t)$ and $\eta(t)$ are given by (6.2) and (6.4) respectively.

Now, using the triangle inequality and (6.1)(i), we obtain

$$
\begin{aligned}
\left\|\widetilde{u_{1 \varepsilon}}+\widetilde{u_{2 \varepsilon}}-u_{1}\right\|_{\mathcal{C}^{0}\left([0, T] ; L^{2}(\Omega)\right)}^{2} & \leq 2\left(\left\|\widetilde{u_{1 \varepsilon}}+\widetilde{u_{2 \varepsilon}}-\Phi\right\|_{\mathcal{C}^{0}\left([0, T] ; L^{2}(\Omega)\right)}^{2}+\left\|\Phi-u_{1}\right\|_{\mathcal{C}^{0}\left([0, T] ; L^{2}(\Omega)\right)}^{2}\right) \\
& \leq 2\left\|\widetilde{u_{1 \varepsilon}}+\widetilde{u_{2 \varepsilon}}-\Phi\right\|_{\mathcal{C}^{0}\left([0, T] ; L^{2}(\Omega)\right)}^{2}+2 \delta^{2} .
\end{aligned}
$$

Moreover, because of the ellipticity condition of $A^{\varepsilon}$ given in (2.8)(i), one has

$$
\frac{1}{2}\left\|\widetilde{u_{1 \varepsilon}}(t)+\widetilde{u_{2 \varepsilon}}(t)-\Phi(t)\right\|_{L^{2}(\Omega)}^{2} \leq \eta_{\varepsilon}(t) .
$$

Hence,

$$
\limsup _{\varepsilon \rightarrow 0}\left\|\widetilde{u_{1 \varepsilon}}+\widetilde{u_{2 \varepsilon}}-u_{1}\right\|_{\mathcal{C}^{0}\left([0, T] ; L^{2}(\Omega)\right)}^{2} \leq c_{1}\left(\limsup _{\varepsilon \rightarrow 0}\left\|\eta_{\varepsilon}\right\|_{\mathcal{C}^{0}([0, T])}+\delta^{2}\right) .
$$

On the other hand,

$$
\nabla u_{1 \varepsilon}-C^{\varepsilon} \nabla u_{1}=\left(\nabla u_{1 \varepsilon}-C^{\varepsilon} \nabla \Phi\right)+C^{\varepsilon}\left(\nabla \Phi-\nabla u_{1}\right) .
$$

By the triangle inequality, (3.4), (6.1)(ii) and the Hölder inequality,

$$
\begin{aligned}
\int_{0}^{T} \| \nabla u_{1 \varepsilon}(t)-C^{\varepsilon} \nabla & u_{1}(t)\left\|_{\left[L^{1}\left(\Omega_{1 \varepsilon}\right)\right]^{n}}^{2} \mathrm{~d} t+\int_{0}^{T}\right\| \nabla u_{2 \varepsilon}(t) \|_{\left[L^{2}\left(\Omega_{2 \varepsilon}\right)\right]^{n}}^{2} \mathrm{~d} t \\
\leq & 2 \int_{0}^{T}\left\|\nabla u_{1 \varepsilon}-C^{\varepsilon} \nabla \Phi\right\|_{\left[L^{1}\left(\Omega_{1 \varepsilon}\right)\right]^{n}}^{2} \mathrm{~d} t+2\left\|C^{\varepsilon}\right\|_{\left[L^{2}\left(\Omega_{1 \varepsilon}\right)\right]^{n^{2}}} \int_{0}^{T}\left\|\nabla \Phi-\nabla u_{1}\right\|_{\left[L^{2}\left(\Omega_{1 \varepsilon}\right)\right]^{n}}^{2} \mathrm{~d} t \\
& +\int_{0}^{T}\left\|\nabla u_{2 \varepsilon}(t)\right\|_{\left[L^{2}\left(\Omega_{2 \varepsilon}\right)\right]^{n}}^{2} \mathrm{~d} t \\
\leq & c_{2} \int_{0}^{T}\left\|\nabla u_{1 \varepsilon}-C^{\varepsilon} \nabla \Phi\right\|_{\left[L^{2}\left(\Omega_{1 \varepsilon}\right)\right]^{n}}^{2} \mathrm{~d} t+c_{3} \delta^{2}+\int_{0}^{T}\left\|\nabla u_{2 \varepsilon}(t)\right\|_{\left[L^{2}\left(\Omega_{2 \varepsilon}\right)\right]^{n}}^{2} \mathrm{~d} t \\
\leq & c_{4}\left(\eta_{\varepsilon}(T)+\delta^{2}\right),
\end{aligned}
$$

where for the last inequality, we used the definition of $\eta_{\varepsilon}$ given in (6.2) and the ellipticity condition of $A^{\varepsilon}$. Therefore,

$$
\limsup _{\varepsilon \rightarrow 0}\left[\left\|\nabla u_{1 \varepsilon}-C^{\varepsilon} \nabla u_{1}\right\|_{L^{2}\left(0, T ;\left[L^{1}\left(\Omega_{1 \varepsilon}\right)\right]^{n}\right)}^{2}+\left\|\nabla u_{2 \varepsilon}\right\|_{L^{2}\left(0, T ;\left[L^{2}\left(\Omega_{2 \varepsilon}\right)\right]^{n}\right)}^{2}\right] \leq c_{4}\left(\limsup _{\varepsilon \rightarrow 0}\left\|\eta_{\varepsilon}\right\|_{\mathcal{C}^{0}([0, T])}+\delta^{2}\right) .
$$


Together with (6.27) and (6.28), we have

$0 \leq \limsup _{\varepsilon \rightarrow 0}\left\{\left\|\widetilde{u_{1 \varepsilon}}+\widetilde{u_{2 \varepsilon}}-u_{1}\right\|_{\mathcal{C}^{0}\left([0, T] ; L^{2}(\Omega)\right)}^{2}+\left\|\nabla u_{1 \varepsilon}-C^{\varepsilon} \nabla u_{1}\right\|_{L^{2}\left(0, T ;\left[L^{1}\left(\Omega_{1 \varepsilon}\right)\right]^{n}\right)}^{2}+\left\|\nabla u_{2 \varepsilon}\right\|_{L^{2}\left(0, T ;\left[L^{2}\left(\Omega_{2 \varepsilon}\right)\right]^{n}\right)}^{2}\right\} \leq c \delta^{2}$.

Since $\delta$ is arbitrary, the conclusion follows.

\subsection{Corrector results for the case $\gamma=1$}

The proof of Theorem 3.9 is based on the following proposition:

Proposition 6.8. Let $\Phi$ and $\Psi$ be in $\mathcal{C}^{\infty}(0, T ; \mathcal{D}(\Omega))$ and set

$$
\begin{aligned}
\beta_{\varepsilon}(t)= & \frac{1}{2}\left\|u_{1 \varepsilon}(t)-\Phi(t)\right\|_{L^{2}\left(\Omega_{1 \varepsilon}\right)}^{2}+\frac{1}{2}\left\|u_{2 \varepsilon}(t)-\Psi(t)\right\|_{L^{2}\left(\Omega_{2 \varepsilon}\right)}^{2} \\
& +\int_{0}^{t} \int_{\Omega_{1 \varepsilon}} A^{\varepsilon}\left(\nabla u_{1 \varepsilon}-C^{\varepsilon} \nabla \Phi\right)\left(\nabla u_{1 \varepsilon}-C^{\varepsilon} \nabla \Phi\right) \mathrm{d} x \mathrm{~d} s+\int_{0}^{t} \int_{\Omega_{2 \varepsilon}} A^{\varepsilon} \nabla u_{2 \varepsilon} \nabla u_{2 \varepsilon} \mathrm{d} x \mathrm{~d} s .
\end{aligned}
$$

Then, under the assumptions of Theorem 3.9, we have

$$
\limsup _{\varepsilon \rightarrow 0}\left\|\beta_{\varepsilon}\right\|_{\mathcal{C}^{0}([0, T])} \leq\|\beta\|_{\mathcal{C}^{0}([0, T])}
$$

where

$$
\begin{aligned}
\beta(t)= & \frac{1}{2} \theta_{1}\left\|u_{1}(t)-\Phi(t)\right\|_{L^{2}(\Omega)}^{2}+\frac{1}{2} \theta_{2}^{-1}\left\|u_{2}(t)-\theta_{2} \Psi(t)\right\|_{L^{2}(\Omega)}^{2} \\
& +\int_{0}^{t} \int_{\Omega} A^{0}\left(\nabla u_{1}-\nabla \Phi\right)\left(\nabla u_{1}-\nabla \Phi\right) \mathrm{d} x \mathrm{~d} s
\end{aligned}
$$

Remark 6.9. In this proposition as in Proposition 6.5, we only have an upper semi-continuity type inequality (see Rem. 6.6) which is likewise sufficient for the main theorem. The outline of the proof of the above proposition is the same as that of Proposition 6.5. However, the part $\beta_{\varepsilon}^{3}$ (see (6.35) below for its definition) of the the energy studied in Step 2 of the proof, requires technical and specific arguments as already encountered in [18] for the corresponding hyperbolic case.

Proof. We closely follow the proof of Proposition 6.5. Only different points will be treated in a detailed manner. To begin with, we decompose $\beta_{\varepsilon}$ into three terms

$$
\beta_{\varepsilon}(t)=\beta_{\varepsilon}^{1}(t)-\beta_{\varepsilon}^{2}(t)+\beta_{\varepsilon}^{3}(t),
$$

where

$$
\begin{aligned}
\beta_{\varepsilon}^{1}(t)= & \frac{1}{2}\|\Phi(t)\|_{L^{2}\left(\Omega_{1 \varepsilon}\right)}^{2}+\frac{1}{2}\|\Psi(t)\|_{L^{2}\left(\Omega_{2 \varepsilon}\right)}^{2}+\int_{0}^{t} \int_{\Omega_{1 \varepsilon}} A^{\varepsilon} C^{\varepsilon} \nabla \Phi C^{\varepsilon} \nabla \Phi \mathrm{d} x \mathrm{~d} s \\
\beta_{\varepsilon}^{2}(t)= & \int_{\Omega_{1 \varepsilon}} u_{1 \varepsilon} \Phi \mathrm{d} x+\int_{\Omega_{2 \varepsilon}} u_{2 \varepsilon} \Psi \mathrm{d} x+\int_{0}^{t} \int_{\Omega_{1 \varepsilon}} A^{\varepsilon} C^{\varepsilon} \nabla \Phi \nabla u_{1 \varepsilon} \mathrm{d} x \mathrm{~d} s+\int_{0}^{t} \int_{\Omega_{1 \varepsilon}} A^{\varepsilon} \nabla u_{1 \varepsilon} C^{\varepsilon} \nabla \Phi \mathrm{d} x \mathrm{~d} s \\
\beta_{\varepsilon}^{3}(t)= & \frac{1}{2}\left\|u_{1 \varepsilon}(t)\right\|_{L^{2}\left(\Omega_{1 \varepsilon}\right)}^{2}+\frac{1}{2}\left\|u_{2 \varepsilon}(t)\right\|_{L^{2}\left(\Omega_{2 \varepsilon}\right)}^{t} \\
& +\int_{0}^{t} \int_{\Omega_{1 \varepsilon}} A^{\varepsilon} \nabla u_{1 \varepsilon} \nabla u_{1 \varepsilon} \mathrm{d} x \mathrm{~d} s+\int_{0}^{t} \int_{\Omega_{2 \varepsilon}} A^{\varepsilon} \nabla u_{2 \varepsilon} \nabla u_{2 \varepsilon} \mathrm{d} x \mathrm{~d} s
\end{aligned}
$$

We continue step by step in getting the limit of each term in (6.32). 
Step 1. In this step, we study the limits of $\beta_{\varepsilon}^{1}$ and $\beta_{\varepsilon}^{2}$. For the first term $\beta_{\varepsilon}^{1}(t)$, following the arguments of the proof of Proposition 5.3 of [15] (as in the proof of Proposition 6.5), it can be shown that

$$
\lim _{\varepsilon \rightarrow 0} \beta_{\varepsilon}^{1}(t)=\frac{1}{2} \theta_{1}\|\Phi(t)\|_{L^{2}(\Omega)}^{2}+\frac{1}{2} \theta_{2}\|\Psi(t)\|_{L^{2}(\Omega)}^{2}+\int_{0}^{t} \int_{\Omega} A^{0} \nabla \Phi \nabla \Phi \mathrm{d} x \mathrm{~d} s \quad \text { in } \mathcal{C}^{0}([0, T])
$$

Now consider the second term $\beta_{\varepsilon}^{2}(t)$ which corresponds to the term $\eta_{\varepsilon}^{2}(t)$ of the previous case. We want to show that

$$
\begin{aligned}
\lim _{\varepsilon \rightarrow 0} \beta_{\varepsilon}^{2}(t)= & \int_{\Omega}\left(\theta_{1} u_{1} \Phi+u_{2} \Psi\right) \mathrm{d} x+\int_{0}^{t} \int_{\Omega} A^{0} \nabla \Phi \nabla u_{1} \mathrm{~d} x \mathrm{~d} s \\
& +\int_{0}^{t} \int_{\Omega} A^{0} \nabla u_{1} \nabla \Phi \mathrm{d} x \mathrm{~d} s \quad \text { in } \mathcal{C}^{0}([0, T]) .
\end{aligned}
$$

The main difference with the previous case lies on the first two terms of $\beta_{\varepsilon}^{2}(t)$ where we need to use convergence (4.5) instead of (4.4) of Theorem 4.8. Indeed, observe that

$$
\begin{aligned}
& \max _{t \in[0, T]} \mid \int_{\Omega_{1 \varepsilon}}\left(u_{1 \varepsilon} \Phi\right.\left.-\theta_{1} u_{1} \Phi\right) \mathrm{d} x+\int_{\Omega_{2 \varepsilon}}\left(u_{2 \varepsilon} \Psi-u_{2} \Psi\right) \mathrm{d} x\left|=\max _{t \in[0, T]}\right| \int_{\Omega}\left(\widetilde{u_{1 \varepsilon}}-\theta_{1} u_{1}\right) \Phi \mathrm{d} x+\int_{\Omega}\left(\widetilde{u_{2 \varepsilon}}-u_{2}\right) \Psi \mathrm{d} x \mid \\
& \leq\left\|\widetilde{u_{1 \varepsilon}}-\theta_{1} u_{1}\right\|_{\mathcal{C}^{0}\left([0, T] ; H^{-1}(\Omega)\right)}\|\Phi\|_{\mathcal{C}^{0}\left([0, T] ; H_{0}^{1}(\Omega)\right)}+\left\|\widetilde{u_{2 \varepsilon}}-u_{2}\right\|_{\mathcal{C}^{0}\left([0, T] ; H^{-1}(\Omega)\right)}\|\Psi\|_{\mathcal{C}^{0}\left([0, T] ; H_{0}^{1}(\Omega)\right)} .
\end{aligned}
$$

Therefore, from (4.5)

$$
\int_{\Omega_{1 \varepsilon}} u_{1 \varepsilon} \Phi \mathrm{d} x+\int_{\Omega_{2 \varepsilon}} u_{2 \varepsilon} \Psi \mathrm{d} x \rightarrow \int_{\Omega}\left(\theta_{1} u_{1} \Phi+u_{2} \Psi\right) \mathrm{d} x \quad \text { in } \mathcal{C}^{0}([0, T]) .
$$

Moreover, the same arguments used to prove (6.10), give

$$
\int_{0}^{t} \int_{\Omega_{1 \varepsilon}} A^{\varepsilon} C^{\varepsilon} \nabla \Phi \nabla u_{1 \varepsilon} \mathrm{d} x \mathrm{~d} s \rightarrow \int_{0}^{t} \int_{\Omega} A^{0} \nabla \Phi \nabla u_{1} \mathrm{~d} x \mathrm{~d} s \quad \text { in } \mathcal{C}^{0}([0, T])
$$

where $u_{1}$ is the solution of the homogenized problem (2.22).

Hence, to prove (6.37), it remains to show that

$$
\int_{0}^{t} \int_{\Omega_{1 \varepsilon}} A^{\varepsilon} \nabla u_{1 \varepsilon} C^{\varepsilon} \nabla \Phi \mathrm{d} x \mathrm{~d} s \rightarrow \int_{0}^{t} \int_{\Omega} A^{0} \nabla u_{1} \nabla \Phi \mathrm{d} x \mathrm{~d} s \quad \text { in } \mathcal{C}^{0}([0, T]) .
$$

The same arguments used to obtain (6.19) and (6.21), give now

$$
\sum_{i=1}^{n} \lim _{\varepsilon \rightarrow 0} \int_{0}^{t} \int_{\Omega}\left(\widetilde{u_{1 \varepsilon}}+\widetilde{u_{2 \varepsilon}}\right) \widehat{w}_{i}^{\varepsilon} \frac{\partial \Phi^{\prime}}{\partial x_{i}} \mathrm{~d} x \mathrm{~d} s=\sum_{i=1}^{n} \int_{0}^{t} \int_{\Omega}\left(\theta_{1} u_{1}+u_{2}\right) x_{i} \frac{\partial \Phi^{\prime}}{\partial x_{i}} \mathrm{~d} x \mathrm{~d} s \quad \forall t \in[0, T]
$$

and

$$
\sum_{i=1}^{n} \lim _{\varepsilon \rightarrow 0} \int_{\Omega}\left(\widetilde{u_{1 \varepsilon}}+\widetilde{u_{2 \varepsilon}}\right)(t) \widehat{w}_{i}^{\varepsilon} \frac{\partial \Phi}{\partial x_{i}}(t) \mathrm{d} x=\sum_{i=1}^{n} \int_{\Omega}\left(\theta_{1} u_{1}+u_{2}\right)(t) x_{i} \frac{\partial \Phi}{\partial x_{i}}(t) \mathrm{d} x \quad \forall t \in[0, T] .
$$


Comparing to limits (6.19) and (6.21), we observe here that we do not replace $u_{2}$ by $\theta_{2} u_{1}$. Furthermore, by Theorem 4.8, (3.15)(ii), (3.6)(ii) and in view of the initial conditions in (2.22),

$$
\begin{aligned}
\lim _{\varepsilon \rightarrow 0} \int_{\Omega}\left(\widetilde{U_{1 \varepsilon}^{0}}+\widetilde{U_{2 \varepsilon}^{0}}\right) \widehat{w}_{i}^{\varepsilon} \frac{\partial \Phi}{\partial x_{i}}(0) \mathrm{d} x & =\int_{\Omega}\left(\theta_{1} U_{1}^{0}+\theta_{2} U_{2}^{0}\right) x_{i} \frac{\partial \Phi}{\partial x_{i}}(0) \mathrm{d} x \\
& =\int_{\Omega}\left(\theta_{1} u_{1}+u_{2}\right)(0) x_{i} \frac{\partial \Phi}{\partial x_{i}}(0) \mathrm{d} x .
\end{aligned}
$$

Hence, as we did to pass to the limit in (6.17),

$$
\begin{aligned}
\sum_{i=1}^{n} \lim _{\varepsilon \rightarrow 0}\left\{\int_{0}^{t}\left\langle u_{1 \varepsilon}^{\prime}, \widehat{w}_{i}^{\varepsilon} \frac{\partial \Phi}{\partial x_{i}}\right\rangle_{\left(V^{\varepsilon}\right)^{\prime}, V^{\varepsilon}} \mathrm{d} s+\int_{0}^{t}\left\langle u_{2 \varepsilon}^{\prime}, x_{i} \frac{\partial \Phi}{\partial x_{i}}\right\rangle_{\left(H^{1}\left(\Omega_{2 \varepsilon}\right)\right)^{\prime}, H^{1}\left(\Omega_{2 \varepsilon}\right)} \mathrm{d} s\right\} & \\
& =\sum_{i=1}^{n} \int_{0}^{t}\left\langle\theta_{1} u_{1}^{\prime}+u_{2}^{\prime}, x_{i} \frac{\partial \Phi}{\partial x_{i}}\right\rangle_{H^{-1}(\Omega), H_{0}^{1}(\Omega)} \mathrm{d} s .
\end{aligned}
$$

Therefore, using (6.11)-(6.15) (which are still valid for $\gamma=1$ ) together with (6.44), yield

$$
\begin{aligned}
\lim _{\varepsilon \rightarrow 0} \int_{0}^{t} \int_{\Omega_{1 \varepsilon}} A^{\varepsilon} \nabla u_{1 \varepsilon} C^{\varepsilon} \nabla \Phi \mathrm{d} x \mathrm{~d} s=\sum_{i=1}^{n}\left\{\int_{0}^{t} \int_{\Omega}\left(\theta_{1} f_{1}+\theta_{2} f_{2}\right) x_{i} \frac{\partial \Phi}{\partial x_{i}} \mathrm{~d} x \mathrm{~d} s+\int_{0}^{t}\left\langle g, x_{i} \frac{\partial \Phi}{\partial x_{i}}\right)\right\rangle_{H^{-1}(\Omega), H_{0}^{1}(\Omega)} \mathrm{d} s \\
\left.-\int_{0}^{t}\left\langle\theta_{1} u_{1}^{\prime}+u_{2}^{\prime}, x_{i} \frac{\partial \Phi}{\partial x_{i}}\right\rangle_{H^{-1}(\Omega), H_{0}^{1}(\Omega)} \mathrm{d} s-\int_{0}^{t} \int_{\Omega} A^{0} \nabla u_{1} \nabla\left(\frac{\partial \Phi}{\partial x_{i}}\right) x_{i} \mathrm{~d} x \mathrm{~d} s\right\} . \quad(6.45)
\end{aligned}
$$

Now, taking $x_{i} \frac{\partial \Phi}{\partial x_{i}}$ as test function in the first and second equations of the homogenized problem (2.22) and integrating by parts, we obtain

$$
\begin{aligned}
\sum_{i=1}^{n}\left\{\int_{0}^{t}\left\langle\theta_{1} u_{1}^{\prime}+u_{2}^{\prime}, x_{i} \frac{\partial \Phi}{\partial x_{i}}\right\rangle_{H^{-1}(\Omega), H_{0}^{1}(\Omega)} \mathrm{d} s+\int_{0}^{t} \int_{\Omega} A^{0} \nabla u_{1} \nabla\left(x_{i} \frac{\partial \Phi}{\partial x_{i}}\right) \mathrm{d} x \mathrm{~d} s\right\} \\
=\sum_{i=1}^{n}\left\{\int_{0}^{t} \int_{\Omega}\left(\theta_{1} f_{1}+\theta_{2} f_{2}\right) x_{i} \frac{\partial \Phi}{\partial x_{i}} \mathrm{~d} x \mathrm{~d} s+\int_{0}^{t}\left\langle g, x_{i} \frac{\partial \Phi}{\partial x_{i}}\right\rangle_{H^{-1}(\Omega), H_{0}^{1}(\Omega)} \mathrm{d} s\right\}
\end{aligned}
$$

which combined with (6.45) gives

$$
\int_{0}^{t} \int_{\Omega_{1 \varepsilon}} A^{\varepsilon} \nabla u_{1 \varepsilon} C^{\varepsilon} \nabla \Phi \mathrm{d} x \mathrm{~d} s \rightarrow \int_{0}^{t} \int_{\Omega} A^{0} \nabla u_{1} \nabla \Phi \mathrm{d} x \mathrm{~d} s \quad \forall t \in[0, T]
$$

To prove that this convergence takes place in $\mathcal{C}^{0}([0, T])$, one argues exactly as we did to show (6.24).

Step 2. It remains to study the term $\beta_{\varepsilon}^{3}(t)$ given in (6.35). Let us first show that

$$
\left\{\beta_{\varepsilon}^{3}\right\} \text { is compact in } \mathcal{C}^{0}([0, T]) \text {. }
$$


To do so, recall that the energy $d_{1 \varepsilon}$ (see (5.1)) associated to (2.14) for $\gamma=1$ is given by

$$
\begin{aligned}
d_{1 \varepsilon}(t)=\frac{1}{2}\left\|u_{1 \varepsilon}(t)\right\|_{L^{2}\left(\Omega_{1 \varepsilon}\right)}^{2}+\frac{1}{2}\left\|u_{2 \varepsilon}(t)\right\|_{L^{2}\left(\Omega_{2 \varepsilon}\right)}^{2} & \int_{0}^{t} \int_{\Omega_{1 \varepsilon}} A^{\varepsilon} \nabla u_{1 \varepsilon} \nabla u_{1 \varepsilon} \mathrm{d} x \mathrm{~d} s \\
& +\int_{0}^{t} \int_{\Omega_{2 \varepsilon}} A^{\varepsilon} \nabla u_{2 \varepsilon} \nabla u_{2 \varepsilon} \mathrm{d} x \mathrm{~d} s+\varepsilon \int_{0}^{t} \int_{\Gamma^{\varepsilon}} h^{\varepsilon}\left|u_{1 \varepsilon}-u_{2 \varepsilon}\right|^{2} \mathrm{~d} \sigma_{x} \mathrm{~d} s .
\end{aligned}
$$

Clearly, from (6.35) we have

$$
\beta_{\varepsilon}^{3}(t)=d_{1 \varepsilon}(t)-\varepsilon \int_{0}^{t} \int_{\Gamma^{\varepsilon}} h^{\varepsilon}\left|u_{1 \varepsilon}-u_{2 \varepsilon}\right|^{2} \mathrm{~d} \sigma_{x} \mathrm{~d} s \quad \forall t \in[0, T] .
$$

From Proposition 5.4, we know that

$$
d_{1 \varepsilon} \rightarrow d_{1} \quad \text { in } \mathcal{C}^{0}([0, T])
$$

Hence, it is enough to show that $\alpha_{\varepsilon}(t)=\varepsilon \int_{0}^{t} \int_{\Gamma^{\varepsilon}} h^{\varepsilon}\left|u_{1 \varepsilon}-u_{2 \varepsilon}\right|^{2} \mathrm{~d} \sigma_{x} \mathrm{~d} s$ is also compact in $\mathcal{C}^{0}([0, T])$. Now, recalling (2.19)(iv) and (2.11), it follows that $\alpha_{\varepsilon}(t)$ is bounded in $H^{1}(0, T)$. Since $H^{1}(0, T) \subset \mathcal{C}^{0}([0, T])$ is a compact injection, we get (6.47).

Let us now prove that

$$
\begin{aligned}
\limsup _{\varepsilon \rightarrow 0} \beta_{\varepsilon}^{3}(t) & \leq \frac{1}{2} \theta_{1}\left\|u_{1}(t)\right\|_{L^{2}(\Omega)}^{2}+\frac{1}{2} \theta_{2}^{-1}\left\|u_{2}(t)\right\|_{L^{2}(\Omega)}^{2}+\int_{0}^{t} \int_{\Omega} A^{0} \nabla u_{1} \nabla u_{1} \mathrm{~d} x \mathrm{~d} s \\
& =d_{1}-c_{h} \theta_{2}^{-1} \int_{0}^{t} \int_{\Omega}\left(\theta_{2} u_{1}-u_{2}\right)^{2} \mathrm{~d} x \mathrm{~d} s \quad \forall t \in[0, T],
\end{aligned}
$$

where $d_{1}(t)$ is given by (5.6), the energy associated with the homogenized problem (2.22).

Due to (6.49), it suffices to show that

$$
\liminf _{\varepsilon \rightarrow 0} \varepsilon \int_{0}^{t} \int_{\Gamma^{\varepsilon}} h^{\varepsilon}\left|u_{1 \varepsilon}-u_{2 \varepsilon}\right|^{2} \mathrm{~d} \sigma_{x} \mathrm{~d} s \geq c_{h} \theta_{2}^{-1} \int_{0}^{t} \int_{\Omega}\left(\theta_{2} u_{1}-u_{2}\right)^{2} \mathrm{~d} x \mathrm{~d} s \quad \forall t \in[0, T] .
$$

To do that, we adapt to the parabolic case the same ideas as in [12,18]. Since in this case $\Gamma$ is assumed of class $C^{2}$, we can apply Lemma 6.4 with $g=h$ and $v_{\varepsilon}=\left(P_{1}^{\varepsilon} u_{1 \varepsilon}-u_{2 \varepsilon}\right)^{2}$, to get

$$
\begin{aligned}
\liminf _{\varepsilon \rightarrow 0} \varepsilon \int_{0}^{t} \int_{\Gamma^{\varepsilon}} h^{\varepsilon}\left|u_{1 \varepsilon}-u_{2 \varepsilon}\right|^{2} \mathrm{~d} \sigma_{x} \mathrm{~d} s & =\liminf _{\varepsilon \rightarrow 0} \varepsilon \int_{0}^{t} \int_{\Gamma^{\varepsilon}} h^{\varepsilon}\left|P_{1}^{\varepsilon} u_{1 \varepsilon}-u_{2 \varepsilon}\right|^{2} \mathrm{~d} \sigma_{x} \mathrm{~d} s \\
& =\liminf _{\varepsilon \rightarrow 0} c_{h} \int_{0}^{t} \int_{\Omega_{2 \varepsilon}}\left|P_{1}^{\varepsilon} u_{1 \varepsilon}-u_{2 \varepsilon}\right|^{2} \mathrm{~d} x \mathrm{~d} s \quad \forall t \in[0, T],
\end{aligned}
$$

where $c_{h}=\frac{1}{\left|Y_{2}\right|} \int_{\Gamma} h(y) \mathrm{d} \sigma_{y}$. Now, by (2.2), (2.19)(i and iii) and Lemma 6.2, we obtain

$$
\begin{aligned}
\left.\widetilde{P_{1}^{\varepsilon} u_{1 \varepsilon}}\right|_{\Omega_{2 \varepsilon}}-\widetilde{u_{2 \varepsilon}} & =\chi_{\Omega_{2 \varepsilon}} P_{1}^{\varepsilon} u_{1 \varepsilon}-\widetilde{u_{2 \varepsilon}} \\
& \rightarrow \theta_{2} u_{1}-u_{2}=\theta_{2}\left(u_{1}-\theta_{2}^{-1} u_{2}\right) \quad \text { weakly in } L^{2}\left(0, T ; L^{2}(\Omega)\right) .
\end{aligned}
$$


Using Lemma 6.3 with $\mathcal{O}_{\varepsilon}=\Omega_{2 \varepsilon}, \chi_{0}=\theta_{2}, v_{\varepsilon}=\left.P_{1}^{\varepsilon} u_{1 \varepsilon}\right|_{\Omega_{2 \varepsilon}}-u_{2 \varepsilon}$ and $v=u_{1}-\theta_{2}^{-1} u_{2}$, we have

$$
\begin{aligned}
\liminf _{\varepsilon \rightarrow 0} c_{h} \int_{0}^{t} \int_{\Omega_{2 \varepsilon}}\left(P_{1}^{\varepsilon} u_{1 \varepsilon}-u_{2 \varepsilon}\right)^{2} \mathrm{~d} \sigma_{x} \mathrm{~d} s & \geq c_{h} \int_{0}^{t} \int_{\Omega} \theta_{2}\left(u_{1}-\theta_{2}^{-1} u_{2}\right)^{2} \mathrm{~d} x \mathrm{~d} s \\
& =c_{h} \theta_{2}^{-1} \int_{0}^{t} \int_{\Omega}\left(\theta_{2} u_{1}-u_{2}\right)^{2} \mathrm{~d} x \mathrm{~d} s \quad \forall t \in[0, T] .
\end{aligned}
$$

Together with (6.52), this gives (6.51) and so (6.50) follows.

Step 3 (conclusion of the proof). From (6.31)-(6.35), together with (6.36), (6.37), (6.47) and (6.50), we deduce that

$$
\left\{\beta_{\varepsilon}\right\} \text { compact in } \mathcal{C}^{0}([0, T]) \quad \text { and } \quad 0 \leq \limsup _{\varepsilon \rightarrow 0} \beta_{\varepsilon}(t) \leq \beta(t), \quad \forall t \in[0, T] .
$$

This gives us the desired result (6.30).

Proof of Theorem 3.9. The proof of Theorem 3.9 will be patterned accordingly to the proof of Theorem 3.4. Let $\delta>0$ be fixed and $\Phi \in \mathcal{C}^{\infty}(0, T ; \mathcal{D}(\Omega))$ be the corresponding function satisfying Lemma 6.1 and associated to the solution $u_{1}$ of $(2.22)$. Moreover, let $\Psi \in \mathcal{C}^{\infty}(0, T ; \mathcal{D}(\Omega))$ such that

$$
\left\|u_{2}-\theta_{2} \Psi\right\|_{\mathcal{C}^{0}\left([0, T] ; L^{2}(\Omega)\right)} \leq \delta .
$$

Note that by (2.8)(ii), Lemma 6.1 and Proposition 6.8,

$$
\begin{aligned}
\limsup _{\varepsilon \rightarrow 0}\left\|\beta_{\varepsilon}\right\|_{\mathcal{C}^{0}([0, T])} \leq & \|\beta\|_{\mathcal{C}^{0}([0, T])} \\
\leq & \frac{1}{2} \theta_{1}\left\|u_{1}-\Phi\right\|_{\mathcal{C}^{0}\left([0, T] ; L^{2}(\Omega)\right)}^{2}+\frac{1}{2} \theta_{2}^{-1}\left\|u_{2}-\theta_{2} \Psi\right\|_{\mathcal{C}^{0}\left([0, T] ; L^{2}(\Omega)\right)}^{2} \\
& +\int_{0}^{T} \int_{\Omega} A^{0}\left(\nabla u_{1}-\nabla \Phi\right)\left(\nabla u_{1}-\nabla \Phi\right) \mathrm{d} x \mathrm{~d} t \\
\leq & \left(\frac{1}{2} \theta_{1}+\frac{1}{2} \theta_{2}^{-1}+\left\|A^{0}\right\|_{\left[L^{\infty}(\Omega)\right]^{n^{2}}}\right) \delta^{2}
\end{aligned}
$$

where $\beta_{\varepsilon}(t)$ and $\beta(t)$ are given by (6.29) and (6.31) respectively.

Now, using the triangle inequality and (6.1)(i), we get

$$
\begin{aligned}
\left\|u_{1 \varepsilon}-u_{1}\right\|_{\mathcal{C}^{0}\left(0, T ; L^{2}\left(\Omega_{1 \varepsilon}\right)\right)}^{2}+ & \left\|u_{2 \varepsilon}-\theta_{2}^{-1} u_{2}\right\|_{\mathcal{C}^{0}\left(0, T ; L^{2}\left(\Omega_{2 \varepsilon}\right)\right)}^{2} \leq \\
\leq & 2\left(\left\|u_{1 \varepsilon}-\Phi\right\|_{\mathcal{C}^{0}\left(0, T ; L^{2}\left(\Omega_{1 \varepsilon}\right)\right)}^{2}+\left\|\Phi-u_{1}\right\|_{\mathcal{C}^{0}\left(0, T ; L^{2}\left(\Omega_{1 \varepsilon}\right)\right)}^{2}\right. \\
& \left.+\left\|u_{2 \varepsilon}-\Psi\right\|_{\mathcal{C}^{0}\left(0, T ; L^{2}\left(\Omega_{2 \varepsilon}\right)\right)}^{2}+\left\|\Psi-\theta_{2}^{-1} u_{2}\right\|_{\mathcal{C}^{0}\left(0, T ; L^{2}\left(\Omega_{2 \varepsilon}\right)\right)}^{2}\right) \\
\leq & 2\left(\left\|u_{1 \varepsilon}-\Phi\right\|_{\mathcal{C}^{0}\left(0, T ; L^{2}\left(\Omega_{1 \varepsilon}\right)\right)}^{2}+\left\|u_{2 \varepsilon}-\Psi\right\|_{\mathcal{C}^{0}\left(0, T ; L^{2}\left(\Omega_{2 \varepsilon}\right)\right)}^{2}\right)+c_{1} \delta^{2} .
\end{aligned}
$$

Furthermore, by the ellipticity of $A^{\varepsilon}$

$$
\frac{1}{2}\left\|u_{1 \varepsilon}(t)-\Phi(t)\right\|_{L^{2}\left(\Omega_{1 \varepsilon}\right)}^{2}+\frac{1}{2}\left\|u_{2 \varepsilon}(t)-\Psi(t)\right\|_{L^{2}\left(\Omega_{2 \varepsilon}\right)}^{2} \leq \beta_{\varepsilon}(t),
$$

so that

$$
\limsup _{\varepsilon \rightarrow 0}\left\{\left\|u_{1 \varepsilon}-u_{1}\right\|_{\mathcal{C}^{0}\left(0, T ; L^{2}\left(\Omega_{1 \varepsilon}\right)\right)}^{2}+\left\|u_{2 \varepsilon}-\theta_{2}^{-1} u_{2}\right\|_{\mathcal{C}^{0}\left(0, T ; L^{2}\left(\Omega_{2 \varepsilon}\right)\right)}^{2}\right\} \leq k_{1}\left(\limsup _{\varepsilon \rightarrow 0}\left\|\beta_{\varepsilon}\right\|_{\mathcal{C}^{0}([0, T])}+\delta^{2}\right) \leq c \delta^{2} .
$$


In a similar manner as in the proof of Theorem 3.4,

$$
\limsup _{\varepsilon \rightarrow 0}\left[\left\|\nabla u_{1 \varepsilon}-C^{\varepsilon} \nabla u_{1}\right\|_{L^{2}\left(0, T ;\left[L^{1}\left(\Omega_{1 \varepsilon}\right)\right]^{n}\right)}^{2}+\left\|\nabla u_{2 \varepsilon}\right\|_{L^{2}\left(0, T ;\left[L^{2}\left(\Omega_{2 \varepsilon}\right)\right]^{n}\right)}^{2}\right] \leq k_{2}\left(\limsup _{\varepsilon \rightarrow 0}\left\|\beta_{\varepsilon}\right\|_{\mathcal{C}^{0}([0, T])}+\delta^{2}\right) \leq c \delta^{2} .
$$

Hence, together with (6.53) and (6.54), we have

$$
\begin{aligned}
0 \leq \limsup _{\varepsilon \rightarrow 0}\left\{\left\|u_{1 \varepsilon}-u_{1}\right\|_{\mathcal{C}^{0}\left(0, T ; L^{2}\left(\Omega_{1 \varepsilon}\right)\right)}^{2}+\right. & \left\|u_{2 \varepsilon}-\theta_{2}^{-1} u_{2}\right\|_{\mathcal{C}^{0}\left(0, T ; L^{2}\left(\Omega_{2 \varepsilon}\right)\right)}^{2} \\
& \left.+\left\|\nabla u_{1 \varepsilon}-C^{\varepsilon} \nabla u_{1}\right\|_{L^{2}\left(0, T ;\left[L^{1}\left(\Omega_{1 \varepsilon}\right)\right]^{n}\right)}^{2}+\left\|\nabla u_{2 \varepsilon}\right\|_{L^{2}\left(0, T ;\left[L^{2}\left(\Omega_{2 \varepsilon}\right)\right]^{n}\right)}^{2}\right\} \leq 2 c \delta^{2} .
\end{aligned}
$$

Since $\delta$ is arbitrary, the conclusion follows.

Acknowledgements. The authors are grateful to the first referee for careful reading, and for a number of helpful suggestions to improve their paper. The second author wishes to express her gratitude to the University of the Philippines for the financial support. The hospitality of the Laboratoire Mathématiques Raphaël Salem of the University of Rouen is also deeply appreciated.

\section{REFERENCES}

[1] J.L. Auriault and H. Ene, Macroscopic modelling of heat transfer in composites with interfacial thermal barrier. International J. Heat Mass Transfer 37 (1994) 2885-2892.

[2] A. Bensoussan, J.L. Lions and G. Papanicolaou, Asymptotic Analysis for Periodic Structures. North-Holland, Amsterdam (1978).

[3] S. Brahim-Otsman, G.A. Francfort and F. Murat, Correctors for the homogenization of the wave and heat equations. J. Math. Pures Appl. 8 (1992) 197-231.

[4] M. Briane, A. Damlamian and P. Donato, H-convergence in Perforated Domains, in Nonlinear Partial Differential Equations and Their Applications - Collège de France Seminar XIII, D. Cioranescu and J.L. Lions Eds., Pitman Research Notes in Mathematics Series 391, Longman, New York, USA (1998) 62-100.

[5] H.S. Carslaw and J.C. Jaeger, Conduction of heat in solids. The Clarendon Press, Oxford, UK (1947).

[6] D. Cioranescu and P. Donato, Homogénéisation du problème de Neumann non homogène dans des ouverts perforés. Asymptot. Anal. 1 (1988) 115-138.

[7] D. Cioranescu and P. Donato, Exact internal controllability in perforated domains. J. Math. Pures Appl. 68 (1989) 185-213.

[8] D. Cioranescu and P. Donato, An Introduction to Homogenization, Oxford Lecture Series in Mathematics and its Applications 17. Oxford Univ. Press, New York, USA (1999).

[9] D. Cioranescu and J. Saint Jean Paulin, Homogenization in open sets with holes. J. Math. Anal. Appl. 71 (1979) $590-607$.

[10] D. Cioranescu and J. Saint Jean Paulin, Homogenization of Reticulated Structures. Springer-Verlag, New York (1999).

[11] D. Cioranescu, P. Donato, F. Murat and E. Zuazua, Homogenization and corrector for the wave equation in domains with small holes. Ann. Scuola Norm. Sup. Pisa Cl. Sci. 2 (1999) 251-293.

[12] P. Donato, Some corrector results for composites with imperfect interface. Rend. Math. Ser. VII 26 (2006) 189-209.

[13] P. Donato and S. Monsurrò, Homogenization of two heat conductors with an interfacial contact resistance. Anal. Appl. 2 (2004) 1-27.

[14] P. Donato and A. Nabil, Approximate controllability of linear parabolic equations in perforated domains. ESAIM: COCV 6 (2001) 21-38.

[15] P. Donato and A. Nabil, Homogenization and correctors for the heat equation in perforated domains. Chin. Ann. Math. B 25 (2004) 143-156.

[16] P. Donato, A. Gaudiello and L. Sgambati, Homogenization of bounded solutions of elliptic equations with quadratic growth in periodically perforated domains. Asymptot. Anal. 16 (1998) 223-243.

[17] P. Donato, L. Faella and S. Monsurrò, Homogenization of the wave equation in composites with imperfect interface: a memory effect. J. Math. Pures Appl. 87 (2007) 119-143.

[18] P. Donato, L. Faella and S. Monsurrò, Correctors for the homogenization of a class of hyperbolic equations with imperfect interfaces. SIAM J. Math. Anal. 40 (2009) 1952-1978.

[19] L. Faella and S. Monsurrò, Memory Effects Arising in the Homogenization of Composites with Inclusions, Topics on Mathematics for Smart Systems. World Sci. Publ., Hackensack, USA (2007) 107-121.

[20] H.K. Hummel, Homogenization for heat transfer in polycrystals with interfacial resistances. Appl. Anal. 75 (2000) $403-424$.

[21] E. Jose, Homogenization of a parabolic problem with an imperfect interface. Rev. Roumaine Math. Pures Appl. 54 (2009) $189-222$. 
[22] J.-L. Lions and E. Magenes, Problèmes aux limites non homogènes et applications, Volume 1. Dunod, Paris, France (1968).

[23] R. Lipton, Heat conduction in fine scale mixtures with interfacial contact resistance . SIAM J. Appl. Math. 58 (1998) 55-72.

[24] R. Lipton and B. Vernescu, Composite with imperfect interface. Proc. Soc. Lond. A 452 (1996) 329-358.

[25] M.L. Mascarenhas, Linear homogenization problem with time dependent coefficient. Trans. Amer. Math. Soc. 281 (1984) 179-195.

[26] S. Monsurrò, Homogenization of a two-component composite with interfacial thermal barrier. Adv. Math. Sci. Appl. 13 (2003) $43-63$.

[27] S. Monsurrò, Erratum for the paper "Homogenization of a two-component composite with interfacial thermal barrier" (in Vol. 13, pp. 43-63, 2003). Adv. Math. Sci. Appl. 14 (2004) 375-377.

[28] S.E. Pastukhova, Homogenization of nonstationary problems in the theory of elasticity on thin periodic structures from the standpoint of the convergence of hyperbolic semigroups in a variable Hilbert space. Sovrem. Mat. Prilozh. 16, Differ. Uravn. Chast. Proizvod. (2004) 64-97 (Russian). Translation in J. Math. Sci. (N. Y.) 133 (2006) 949-998.

[29] R.E. Showalter, Distributed microstructure models of porous media, in Flow in porous media (Oberwolfach (1992)), J. Douglas and U. Hornung Eds., Internat. Ser. Numer. Math. 114, Birkhäuser, Basel, Switzerland (1993) 155-163.

[30] L. Tartar, Cours Peccot. Collège de France, France, unpublished (1977).

[31] L. Tartar, Quelques remarques sur l'homogénéisation, in Functional Analysis and Numerical Analysis, Proc. Japan-France Seminar 1976, Japanese Society for the Promotion of Science (1978) 468-482.

[32] L. Tartar, Memory effects and homogenization. Arch. Rational Mech. Anal. 3 (1990) 121-133. 